\title{
Microgel technology for improved beta cell transplantation
}

Sieger Henke 


\section{Microgel technology for improved beta cell transplantation}

Sieger Henke

2016 


\section{Members of the Graduation Committee}

\section{Chairman:}

Prof. dr. ir. J.W.M. Hilgenkamp

\section{Promotor:}

Prof. dr. H.B.J. Karperien (University of Twente)

\section{Co-promotors:}

Dr. J.C.H. Leijten (University of Twente)

Dr. ing. A.A. van Apeldoorn (University of Twente)

\section{Members:}

Prof. dr. P.C.J.J. Passier (University of Twente)

Prof. dr. D. Stamatialis (University of Twente)

Prof. dr. E.J.P. de Koning (Leiden University Medical Center / Hubrecht Institute)

Prof. dr. M. Gotthardt (Radboud University Medical Center)

Dr. F. Carlotti (Leiden University Medical Center)

Prof. dr. L.W.M.M. Terstappen (University of Twente)

Microgel technology for improved beta cell transplantation 2016, Sieger Henke

The research described in this thesis was supported by the Dutch Diabetes Cell Therapy Initiative (DCTI), and the Dutch Diabetes Research Foundation.

This publication was supported by the Netherlands Society for Biomaterials and Tissue Engineering (NBTE).

ISBN: 978-90-365-4204-3

Copyright: Sieger Henke, 2016, The Netherlands. Neither this thesis nor its parts may be reproduced without written permission of the author.

Printed by: Ipskamp, Enschede, The Netherlands 


\title{
DISSERTATION
}

\author{
to obtain \\ the degree of doctor at the University of Twente, \\ on the authority of the rector magnificus, \\ prof. dr. H. Brinksma, \\ on account of the decision of the graduation committee, \\ to be publicly defended \\ on Friday, November $11^{\text {th }} 2016$ at 16.45
}

by

Sieger Jakob Henke

Born on November $6^{\text {th }}, 1986$

in Beuningen, The Netherlands 
This dissertation has been approved by:

Prof. dr. H.B.J. Karperien (Promotor)

Dr. J.C.H. Leijten (Co-promotor)

Dr. ing. A.A. van Apeldoorn (Co-promotor) 


\section{Contents}

Chapter 1 Introduction \& thesis outline

Page

1

Chapter 2 Enzymatic crosslinking of polymer conjugates is superior over ionic or UV crosslinking for the on-chip production of cell-laden microgels

Chapter 3 The stiffness and composition of micro hydrogels independently control mesenchymal stromal cell differentiation in the osteogenic lineage

Chapter 4 Proliferation restrictive micro-environments for beta cell encapsulation

Chapter 5 Biofabrication of core-shell hydrogels as microbioreactors for controlled cell aggregation

Chapter 6 Centering single cells in spherical microgels by uncoupling on-chip emulsification and enzymatic crosslinking using hydrogen peroxide diffusion

Chapter 7 General discussion

Summary

Samenvatting

List of publications

Curriculum Vitae

Acknowledgements 

Chapter 1

Introduction \& thesis outline 


\subsection{General introduction on diabetes}

Diabetes mellitus affects approximately $8.2 \%$ of adults between 20 and 79 worldwide, and the estimated annual global health expenditure associated with this disease was between 612 and 1099 billion US dollars ${ }^{[1]}$. The prevalence is expected to rise even further ${ }^{[2]}$. The three main types of diabetes are type 1 , type 2 and gestational diabetes.

Type 1 diabetes is caused by the autoimmune destruction of the insulinproducing beta cells, located in the islets of Langerhans in the pancreas. As a consequence no insulin is produced and released. Insulin is a hormone that is required to transport glucose from the blood into the cells. As a consequence cells cannot take up glucose anymore and blood glucose levels in the blood rise. These high levels cause damage to all kinds of tissues, such as the vasculature, kidney and heart. Frequent insulin injections or beta cell replacement is necessary to restore the glucose homeostasis. Type 1 diabetes usually affects children or young adults, who will have to cope with this disease for the rest of their lives, as no definitive cure is available.

Diabetes type 2 occurs most in adults and is the most frequent type of diabetes. In contrary to diabetes type 1 , the beta cells in the pancreas are still functional, but the body is resistant to the produced insulin, resulting in high glucose concentration in the blood. The exact mechanisms resulting in type 2 diabetes are unknown, but commonly assumed is that an unhealthy lifestyle with few physical activity, overweight and a poor diet are usually the cause. Frequently, a change of lifestyle can already (partially) reverse the diabetes, and no insulin treatment is necessary.

Gestational diabetes, or diabetes mellitus in pregnancy most frequently occurs from the $24^{\text {th }}$ week of pregnancy. Normally it disappears after the pregnancy, but women who had gestational diabetes are at risk for developing diabetes type $2^{[3]}$.

\subsection{Treatment of diabetes type 1}

Diabetes type 1 is a chronic disease, and can only be cured by restoring or replacing the function of the beta cells. The first "cell therapy" for diabetes started even before insulin was discovered, as in 1893 sheep pancreas pieces were implanted into a 15 year old boy suffering from diabetes ${ }^{[4]}$. The boy died a 
few days later, but the idea of replacing pancreas or beta cell function was born. Insulin therapy was first applied in $1921^{[5]}$. The widespread availability of insulin therapy transformed diabetes type 1 from a deadly disease to a chronic disease that requires careful management and daily insulin injections. However, diabetics are threatened by numerous long-term complications like retinopathy and kidney failure contributing to a reduced life expectancy ${ }^{[6]}$.

As already pioneered in 1893, transplantation of insulin producing cells is still a very promising treatment to type 1 diabetes. Current success is achieved with transplantation of an entire donor pancreas, or transplantation of isolated donor islets of Langerhans. These therapies can be called successful, as they result in more stable glucose levels and less insulin dependency ${ }^{[7]}$. However, the need of immunosuppression and the limited availability of donor pancreases makes these treatments far from ideal and the availability very limited. As the burden of immunosuppression is usually too high to justify for a pancreas or islet transplantation alone, these transplantations are mainly performed in patients that already receive immunosuppression for another transplant, usually a kidney [7]. Until better options are available, islet transplantation is a good treatment for a specific group of patients with type 1 diabetes at high risk for life-threatening hypoglycemia. However, other sources of insulin producing cells are needed for the treatment of the majority of the patients.

\subsection{Beta cell sources}

As described above, the current beta cell source for transplantations is a donor pancreas from which the islets of Langerhans are isolated. As other sources of beta cells are needed, there is the search for alternatives. The first explored alternative cell source for transplantation was a sheep pancreas ${ }^{[4]}$. Nowadays pig pancreases are under investigation for use in beta cell replacement ${ }^{[8]}$. However, the use of non-human materials is controversial and still poses technical challenges ${ }^{[8,9]}$.

Several types of stem cells have a great promise to differentiate into functional beta cells and be a future replenishable source of beta cells for the treatment of diabetes type $1^{[10]}$. Stem cells are characterized by their extensive or unlimited capacity to proliferate, and their ability to differentiate into a specialized cell. 


\subsubsection{Stem cells}

For a long time, embryonic stem cells (ESCs), harvested from left-over embryos for in vitro fertilization (ivf), were the most promising source of stem cell derived beta cells, and successful differentiation protocols have been reported [11]. However, embryonic stem cells are allogenic, resulting in the need of immunoprotection of the transplanted cells or immunosuppression to protect the transplanted cells from rejection by the recipient's immune system ${ }^{[12]}$.

The invention of induced pluripotent stem cells (iPSCs) made the great promise of an unlimited source of autogenic stem cells, in contrast with ESCs which will be allogenic. iPSCs are made by reprogramming adult cells into embryonic stem cell like cells ${ }^{[13]}$. Pagliuca and coworkers have successfully differentiated these iPSCs into functional, insulin secreting beta cells ${ }^{[14]}$. They report their protocol is scalable to produce at least hundreds of millions of cells in vitro. This breakthrough could lead to a cure for diabetes type 1 , however there are still enough hurdles to take. To name a few, the differentiation protocols have to be optimized and scaled to a very large scale, all components have to be made clinical grade and the price of whole procedure has to be low enough to be paid for by insurance companies. Most importantly, the final graft has to be safe, mitigating all the risks associated with stem cells.

\subsubsection{Risks of using stem cells}

One of the properties of pluripotent and embryonic stem cells is their renewal capacity. This property inherits an important risk. When undifferentiated cells are injected in an animal, they can continue their renewal in an uncontrolled way, and spontaneously differentiate into all kinds of tissues, forming a teratocarcinoma ${ }^{[15]}$. As current differentiation protocol cannot guarantee $100 \%$ differentiated cells, this is a serious risk when using embryonic stem cells or iPSC based therapy ${ }^{[15]}$.

\subsection{Tissue engineering approach}

The most easy and straightforward way of transplanting cells is injecting cells in suspension. However, this is only possible in few places and for few tissues. When transplanting a large amount of cells, the majority of the cells will probably clump together, causing diffusion limitations to the core of the clumps and ultimately massive cell death. 
Thus when transplanting cells, the right environment for the cells is important. As curing a patient with type 1 diabetes requires roughly $10^{9}$ beta cells [12], and the cells need to be as close as possible to vascularization in a very short time, not only for the cells to survive. Namely, as a good glucose homeostasis requires a fast reaction of the beta cells, and thus adequate vascularization of the graft is essential. Thus the transplanting environment should allow for fast vascularization [16]. Also in general, vascularization is the main key when transplanting cells ${ }^{[17]}$.

Thus this approach, and many more in tissue engineering and regenerative medicine, will need some kind of supporting material for the transplanted cells ${ }^{[18]}$. The use of a supporting material with cells, is called tissue engineering. The United States National Institute of Health goal of tissue engineering is "to assemble functional constructs that restore, maintain or improve damaged tissues or whole organs" ${ }^{[19]}$. These functional constructs consist of cells, supported by biomaterials and growth factors.

In order to create a successful tissue engineering construct, it is essential to mimic the complexity of the native tissue ${ }^{[20]}$. This comprises the assembly of different micro and nanostructures into a functional construct ${ }^{[21]}$. Hydrogels and micro hydrogels are excellent building blocks for such an approach.

\subsubsection{Hydrogels}

Hydrogels are hydrophilic crosslinked polymers which hold large amounts of water in their polymer network. The hydrophilic water-rich environment resembles the composition of the human body and is a good matrix to support cells ${ }^{[22]}$. The material composition of the hydrogel can be divided into two types, natural polymers like alginate, hyaluronic acid, gelatin and dextran, and synthetic polymers like poly ethylene glycol (PEG) and poly hydroxyethyl methacrylate (pHEMA).

To make a water-retaining network from loose polymer chains, they have to be crosslinked together. There are two main types of gel crosslinking, chemical crosslinking, where a chemical reaction leads to a covalent bond, and physical crosslinking, where the polymer chains are kept together by physical forces like ionic interactions or hydrogen bonds ${ }^{[23]}$. When encapsulating living cells, the crosslinking of the hydrogel should be performed under cytocompatible, and thus mild, physiological conditions. Commonly used crosslinking methods that are 
cytocompatible are supramolecular- (including temperature induced and ionic), photo-, and enzymatic crosslinking. For example, PEG hydrogels are often made by conjugating acrylate groups that can be photo crosslinked, alginate gels are made by ionic crosslinking with $\mathrm{Ca}^{2+}$ and dextran hydrogels can be made by adding tyramine groups that can be enzymatic crosslinked using horseradish peroxidase and hydrogen peroxide. In chapter 2 of this thesis we investigated the pros and cons of these three crosslinking methods for the encapsulation of cells.

\subsubsection{Microgel}

Microgel technology is the generation of sub millimeter hydrogels particles. Numerous methods are available to produce microgels, all based on the generation of droplets that are subsequently crosslinked into hydrogel particles. These droplets can be generated by spraying devices in which droplet formation is either controlled by air, piezo or electric fields, or by emulsification. This emulsification can be 'uncontrolled' by stirring oil in water. This results in the production of polydisperse droplets. When using microfluidic devices it is possible to obtain monodisperse droplets, which is advantageous due to reduction in variability in size of the constructs.

\subsection{Microfluidics}

Droplet microfluidics is the technique of generating droplets in a microfluidic channel (sub millimeter channels). By forming droplets of polymer conjugates and subsequent crosslinking of these droplets, microgels can be generated. Microgels are already used for a long time in immunoprotective islet encapsulation ${ }^{[24]}$, however these gels are usually made with an electrostatic droplet generator. These gels are relatively large, usually around $500 \mu \mathrm{m}$, and not uniform in size.

Microfluidic devices for producing droplets can be made with various techniques and materials. A fast, highly reproducible and easy way to make microfluidic devices is using soft lithography techniques ${ }^{[25]}$. With this technique microfluidic channels are made by replica molding of silicon rubber from a silicon mold which has been made using lithography techniques. As devices made with soft lithography have some limitations in design and size, for this thesis also full 3D microfluidic devices were used. These devices are build using tubing and capillaries, and are very useful to produce fairly large droplets of over $150 \mu \mathrm{m}$ in 
a controlled way. A disadvantage of full 3D microfluidic devices are limitations in design variations compared to the use of soft lithography.

\subsubsection{Crosslinking of hydrogel droplets}

After a droplet of hydrogel precursor is formed in a microfluidic device, the droplet has to be crosslinked to form a microgel. As described in paragraph 1.4.1 of this chapter, there are numerous crosslinking approaches to crosslink polymer conjugates into a hydrogel network. Crosslinking microgels adds some complexity to the microfluidic system. In many cases the crosslinking agent is potentially harmful for the cells, and thus all droplets should be crosslinked without unnecessary exposure of the crosslinking agent to the encapsulated cells.

A relatively easy way to crosslink micro droplets would be collecting the emulsion and subsequently perform the crosslinking in bulk. However, this has several downsides. The most important downside is the stability of the emulsion. In order to collect and store an emulsion with cell-laden droplets, very strong surfactants are necessary to prevent merging of droplets. Such surfactants are subsequently very difficult to remove. To circumvent this drawback, in this thesis we have focused on the development of methods for crosslinking of droplets onchip, thus the surfactant only has to aid in droplet formation and is required very short term stabilization of the emulsion only. 


\subsection{Aim and outline of this thesis}

In this thesis, we describe the explored and developed strategies to encapsulate (beta) cells in micro hydrogels, with the special aim of contributing to curing diabetes type 1 in the future.

Chapter 2, "Enzymatic crosslinking of polymer conjugates is superior over ionic or UV crosslinking for the on-chip production of cell-laden microgels", describes the development of a microfluidic platform to produce cell encapsulating microgels using either photo, ionic or enzymatic crosslinking. We aimed at selecting the best crosslinking method for encapsulating cells in microgels on-chip, by comparing photo crosslinking of polyethylene-glycol diacrylate (PEGDA), ionic crosslinking of alginate and enzymatic crosslinking of dextran-tyramine conjugates (Dex-TA) for production of cell laden microgels. Mechanical properties, cells survival and metabolic activity of encapsulated human mesenchymal stromal cells (hMSCs) were tested.

In chapter 3, "The stiffness and composition of micro hydrogels independently control mesenchymal stromal cell differentiation in the osteogenic lineage", the platform for production of enzymatically crosslinked Dex-TA microgels was further explored and expanded by combining it with hyaluronic acid, and tuning the stiffness of the microgels. Stiffness and composition of the matrix around cells is known to influence cell behavior. To investigate the influence of hyaluronic acid and the variation in stiffness on encapsulated cells, hMSCs were encapsulated in different types of gels and their differentiation towards the adipogenic and osteogenic lineage was studied.

In chapter 4, "Proliferation restrictive micro-environments for beta cell encapsulation", the platform from chapter 3 was applied on a beta cell model in order to form a proliferation restrictive environment for transplantation of (iPSC derived) beta cells. As differentiation protocols don't reach $100 \%$ differentiation, stem cell derived cells have the inherent risk of uncontrolled cell proliferation. We tested the survival and proliferation of in microgels encapsulated cells in vitro and in an in vivo model using the MIN6 beta cell line.

In chapter 5, "Biofabrication of core-shell hydrogels as microbioreactors for controlled cell aggregation", a platform to produce enzymatically crosslinked core-shell microgels of Dex-TA and PEG-TA was developed. These microgels can be used to efficiently form aggregates of cells in confined microenvironment. 
These aggregates are very useful for high throughput screening of drugs, the differentiation of stem cells and in situ delivery of therapeutic factors such as insulin.

In chapter 6, "Centering single cells in spherical microgels by uncoupling on-chip emulsification and enzymatic crosslinking using hydrogen peroxide diffusion", we scaled down the enzymatically crosslinked microgels to single cell encapsulating microgels, with a size not much bigger than a single cell. As the techniques we used before to induce the crosslinking in microgels did not suffice to produce these sub $50 \mu \mathrm{m}$ gels, a new chip was developed and characterized to produce these microgels. One of the crosslinking components is supplied via diffusion through a PDMS wall in a dedicated PDMS chip. In this system, the single cells are centered in the microgel, which is important to prevent cell escape and ensure uniform 3D culture conditions.

Finally, chapter 7 discusses the results and highlights further improvements and application of microgel technology for the treatment of diabetes. 


\section{References}

[1] J. da Rocha Fernandes, K. Ogurtsova, U. Linnenkamp, L. Guariguata, T. Seuring, P. Zhang, D. Cavan, and L. E. Makaroff, "IDF Diabetes Atlas estimates of 2014 global health expenditures on diabetes", Diabetes Research and Clinical Practice, vol. 117, pp. 48-54, 2016.

[2] Wild, "Estimates for the year 2000 and projections for 2030", World Health, vol. 27, no. 5, pp. 1047-1053, 2004.

[3] L. Bellamy, J.-P. Casas, A. D. Hingorani, and D. Williams, "Type 2 diabetes mellitus after gestational diabetes: a systematic review and metaanalysis.", Lancet, vol. 373, no. 9677, pp. 1773-1779, 2009.

[4] P. Williams, "Notes on diabetes treated with extract and by grafts of sheep's pancreas", British Medical Journal, no. 2, pp. 1303-1304, 1894.

[5] F. G. Banting and C. H. Best, "The internal secretion of the pancreas", The Journal of Laboratory and Clinical Medicine, vol. 7, no. 5, pp. 251-266, 1922.

[6] D. Daneman, "Type 1 diabetes", Lancet, vol. 367, no. 9513, pp. 847-858, 2006.

[7] American Diabetes Association, "Pancreas and Islet Transplantation in Type 1 Diabetes", Diabetes Care, vol. 29, no. 4, pp. 935-935, 2006.

[8] D. J. Van Der Windt, R. Bottino, G. Kumar, M. Wijkstrom, H. Hara, M. Ezzelarab, B. Ekser, C. Phelps, N. Murase, A. Casu, D. Ayares, F. G. Lakkis, M. Trucco, and D. K. C. Cooper, "Clinical islet xenotransplantation: How close are we?", Diabetes, vol. 61, no. 12, pp. 3046-3055, 2012.

[9] J. L. Platt, "Xenotransplantation: New risks, new gains", Nature, vol. 407, no. 6800, pp. 27-30, 2000.

[10] C. Aguayo-Mazzucato and S. Bonner-Weir, "Stem cell therapy for type 1 diabetes mellitus", Nature Reviews Endocrinology, vol. 6, no. 3, pp. 139148, 2010.

[11] B. Johannesson, L. Sui, D. O. Freytes, R. J. Creusot, and D. Egli, "Toward beta cell replacement for diabetes.", The EMBO journal, vol. 34, no. 7, pp. 841-55, 2015.

[12] B. Soria, B. R. Gauthier, F. Martín, J. R. Tejedo, F. J. Bedoya, A. Rojas, and A. Hmadcha, "Using stem cells to produce insulin.", Expert opinion on biological therapy, vol. 15, no. 10, pp. 1469-89, 2015.

[13] K. Takahashi and S. Yamanaka, "Induction of Pluripotent Stem Cells from Mouse Embryonic and Adult Fibroblast Cultures by Defined Factors", Cell, vol. 126, no. 4, pp. 663-676, 2006.

[14] F. W. Pagliuca, J. R. Millman, M. Gürtler, M. Segel, A. Van Dervort, J. H. Ryu, Q. P. Peterson, D. Greiner, and D. A. Melton, "Generation of Functional Human Pancreatic $\beta$ Cells In Vitro", Cell, vol. 159, no. 2, pp. 428-439, 2014. 
[15] S. G. Hong, T. Winkler, C. Wu, V. Guo, S. Pittaluga, A. Nicolae, R. E. Donahue, M. E. Metzger, S. D. Price, N. Uchida, S. A. Kuznetsov, T. Kilts, L. Li, P. G. Robey, and C. E. Dunbar, "Path to the clinic: assessment of iPSCbased cell therapies in vivo in a nonhuman primate model.", Cell reports, vol. 7, no. 4, pp. 1298-309, 2014.

[16] N. Sakata, T. Aoki, G. Yoshimatsu, H. Tsuchiya, T. Hata, Y. Katayose, S. Egawa, and M. Unno, "Strategy for clinical setting in intramuscular and subcutaneous islet transplantation", Diabetes/Metabolism Research and Reviews, vol. 30, no. 1, pp. 1-10, 2014.

[17] S. Dimmeler, S. Ding, T. A. Rando, and A. Trounson, "Translational strategies and challenges in regenerative medicine.", Nature medicine, vol. 20, no. 8, pp. 814-21, 2014.

[18] V. F. M. Segers and R. T. Lee, "Biomaterials to enhance stem cell function in the heart", Circulation Research, vol. 109, no. 8, pp. 910-922, 2011.

[19] "National Institutes of Health", 2016. [Online]. Available: https://www.nibib.nih.gov/science-education/science-topics/tissueengineering-and-regenerative-medicine\#1156. [Accessed: 01-Jan-2016].

[20] J. Leijten, Y. C. Chai, I. Papantoniou, L. Geris, J. Schrooten, and F. P. Luyten, "Cell based advanced therapeutic medicinal products for bone repair: Keep it simple?", Advanced Drug Delivery Reviews, vol. 84, pp. 30-44, 2015.

[21] J. Leijten, J. Rouwkema, Y. S. Zhang, A. Nasajpour, M. R. Dokmeci, and A. Khademhosseini, "Advancing Tissue Engineering: A Tale of Nano-, Micro-, and Macroscale Integration", Small, vol. 12, no. 16, pp. 2130-2145, 2016.

[22] L. S. Moreira Teixeira, J. Feijen, C. a. van Blitterswijk, P. J. Dijkstra, and M. Karperien, "Enzyme-catalyzed crosslinkable hydrogels: Emerging strategies for tissue engineering", Biomaterials, vol. 33, no. 5, pp. 12811290, 2012.

[23] W. E. Hennink and C. F. van Nostrum, "Novel crosslinking methods to design hydrogels", Advanced Drug Delivery Reviews, vol. 64, no. SUPPL., pp. 223-236, 2012.

[24] F. Lim and A. Sun, "Microencapsulated islets as bioartificial endocrine pancreas", Science, vol. 210, no. 4472, pp. 908-910, 1980.

[25] J. C. McDonald and G. M. Whitesides, "Poly(dimethylsiloxane) as a Material for Fabricating Microfluidic Devices", Accounts of Chemical Research, vol. 35, no. 7, pp. 491-499, 2002. 


\section{Chapter 2}

Enzymatic crosslinking of polymer conjugates is superior over ionic or UV crosslinking for the on-chip production of cell-laden microgels

Sieger Henke, Jeroen Leijten, Evelien Kemna, Martin Neubauer, Andreas Fery, Albert van den Berg, Aart van Apeldoorn, Marcel Karperien 


\section{Abstract}

Cell-laden micrometer-sized hydrogels (microgels) hold great promise for improving high throughput ex-vivo drug screening and engineering biomimetic tissues. Microfluidics is a powerful tool to produce microgels. However, only a limited amount of biomaterials have been reported to be compatible with onchip microgel formation. Moreover, these biomaterials are often associated with mechanical instability, cytotoxicity, and cellular senescence. To resolve this challenge, we have explored dextran-tyramine as a novel biomaterial for on-chip microgel formation. In particular, we compared dextran-tyramine with two commonly used biomaterials namely PEGDA and alginate, which crosslink through enzymatic reaction, UV polymerization, and ionic interaction, respectively. Human mesenchymal stem cells (hMSCs) encapsulated in dextrantyramine microgels demonstrated significantly higher (95 \%) survival as compared to alginate (81 \%) and PEGDA (69\%). Long-term cell cultures demonstrated that hMSCs in PEGDA microgels became senescent after 7 days. Alginate microgels dissolved within 7 days due to $\mathrm{Ca}^{2+}$ loss. In contrast, dextrantyramine based microgels remained stable, sustained hMSCs metabolic activity, and permitted for single-cell level analysis for at least 28 days of culture. In conclusion, enzymatically crosslinking dextran-tyramine conjugates represent a novel biomaterial class for the on-chip production of cell-laden microgels, which possesses unique advantages as compared to the commonly used UV and ionic crosslinking biomaterials. 


\section{Introduction}

The cellular microenvironment strongly controls the function and fate of cells. Consequently, removing cells from their natural environment drastically and progressively alters a cell's phenotype and capacity to perform its native function ${ }^{[1-3]}$. Encapsulating cells in engineered biomimetic environments using instructive biomaterials therefore represents a powerful tool to control cell behavior ${ }^{[4,5]}$. One of the most common methods for embedding cells in a 3D environment is through hydrogels encapsulation ${ }^{[6]}$. Hydrogel design parameters currently are focused on balancing the biomaterial's physical properties including e.g. shape, stiffness, porosity, and degradation rate and chemical cues including presentation of specific cues such as matrix and growth factors. However, the demands in hydrogel design from hydrogel-host perspective and hydrogelencapsulated cells perspective are often conflicting. This can be resolved by microencapsulating cells first in an instructive micrometer-sized biomaterial, which in turn is incorporated in a second biomaterial with distinct properties.

Unfortunately, only a limited amount of biomaterials have been explored for microencapsulation ${ }^{[7-11]}$. These biomaterials are typically associated with poor mechanical properties, significant amounts of cell death, and insufficient levels of cell stimulation. This has resulted in suboptimal outcomes in short term culture results and incompatibility with long term cultures.

Of the explored materials, alginate has received a substantial amount of attention ${ }^{[12]}$. Alginate is a polysaccharide extracted from the cell walls of brown algae that is typically crosslinked using divalent ions such as $\mathrm{Ca}^{2+}$. However, $\mathrm{Ca}^{2+}$ is lost over time due to diffusion, leading to a progressive uncontrolled decrease in stiffness and inevitable structural failure of the microgel ${ }^{[13]}$. Another commonly explored biomaterial for microfluidic microgel formation is the synthetic polymer polyethylene-glycol (PEG) ${ }^{[8]}$. Fast, on-chip crosslinking of PEG microgels requires photo crosslinking e.g. PEG diacrylate (PEGDA), as other crosslinking methods like thiol-ene click chemistry take too much time to crosslink on-chip only ${ }^{[14]}$. However, this requires high power UV irradiation, which is correlated with suboptimal cell survival and potential genetic damage ${ }^{[15]}$. Next to these, several other less commonly explored biomaterials have been reported for the production of cell-laden microgels, such as thiol conjugated hyaluronic acid ${ }^{[14]}$. However, these materials have crosslinking times ranging from minutes to hours. 
This requires off chip crosslinking, for which droplets need to be stabilized by powerful surfactants ${ }^{[14,16]}$. These approaches demand additional time consuming steps to be compatible with down-stream processes. Thus, there is a clear need for an on-chip crosslinking biomaterial that allows for high cell survival, does not induces genetic damage, and allows for facile chemical modification to allow the fabrication of biomimetic microenvironments.

Here, we report on dextran-tyramine conjugates (Dex-TA) as a novel biomaterial for microencapsulation ${ }^{[17]}$. Dextran is a natural polymer with excellent biocompatibility. Once conjugated to tyramine, the resulting polymer conjugate can be enzymatically crosslinked using horseradish peroxidase in the presence of hydrogen peroxide. It is potentially ideal for on-chip microgel formation, due to its extremely fast, yet cell friendly gelation. Moreover, numerous modifications are available to tune Dex-TA's bioactivity, degradability, and mechanical stiffness ${ }^{[18,19]}$.

In order to make a direct comparison between photo, ionic and enzymatic crosslinking, we developed a novel universal microfluidic chip. This device allows for the controlled crosslinking of each of the three mentioned methods. Using this platform, we have compared microencapsulation of human mesenchymal stem cells (hMSCs) in PEGDA, alginate and Dex-TA microgels, in long-term cell cultures. In short, we have shown that on-chip enzymatically crosslinking of cellladen microgels has superior properties in terms of cell survival, metabolic activity and stability in both short and long-term cell cultures, as compared to ionically crosslinked alginate and UV crosslinked PEGDA. 


\section{Materials and Methods}

\section{Microfluidic chip production}

Microfluidic devices were made using a standard soft lithography process. In short, microfluidic channels were designed using CAD software (Clewin, Wieweb software) and patterned onto a chromium photomask. A layer of SU-8 polymer $(70 \mu \mathrm{m})$ was spun on a silicon wafer and exposed to UV through the photomask, and was subsequently developed to produce the mold. Polydimethylsiloxane (PDMS, Sylgard 184, Dow Corning) was mixed in a 10:1 base:curing agent ratio, casted on the mold and cured. The PDMS was peeled from the mold, holes were punched to create inlets and outlets, and the PDMS was bound to a glass slide using oxygen plasma. A schematic representation, a microphotograph, and scanning electron micrographs of key elements of the universal chip design are shown in Figure 1.

\section{Cell Culture}

The use of patient material was approved by the local ethical committee of the Medisch Spectrum Twente, and informed written consent was obtained for all samples. Human mesenchymal stem cells (hMSCs) were isolated from bone marrow as described previously ${ }^{[20]}$. The cells were cultured in Alfa-MEM (Invitrogen), supplemented with heat-inactivated FBS (10\%, Lonza), L-glutamine (2 mM, Invitrogen), ascorbic acid (0,2 mM, Sigma-Aldrich), basic fibroblast growth factor (1 ng/ml, ISOKine bFGF, Neuromics), and penicillin (100 U/ml) and streptomycin $(100 \mu \mathrm{g} / \mathrm{ml}$, Invitrogen). Cells were kept in a humidified environment with $5 \% \mathrm{CO}_{2}$ and used from passage 3 to 5 . 

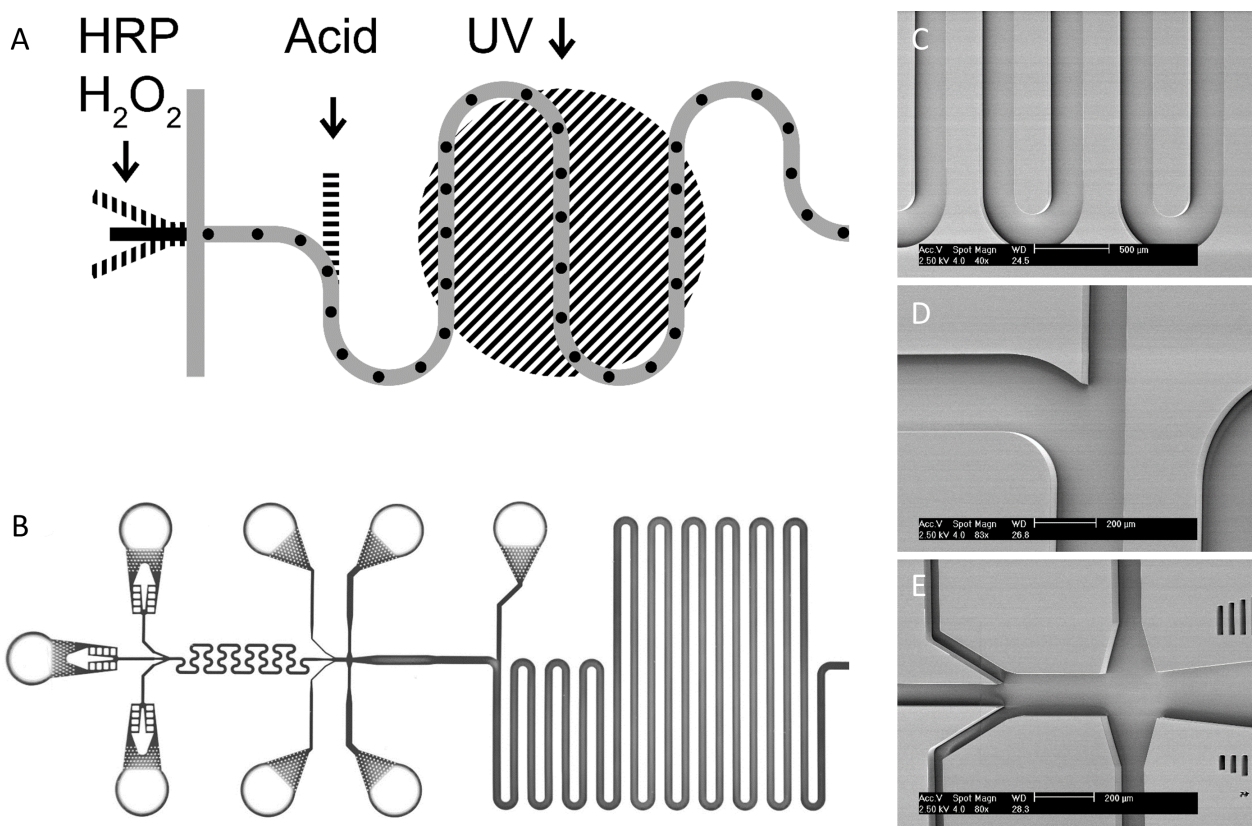

Figure 1. (A) Schematic overview of the universal microfluidic chip used for production of photo crosslinked PEGDA, ionic crosslinked alginate and enzymatically crosslinked Dex-TA microgels. Dashed areas indicate where photo, ionic and enzymatic crosslinking are initiated. (B) Photograph of the universal microfluidic chip containing a black dye to visualize inlets on the left, incubation channel and outlet on the right. Scanning electron micrographs were made of $(C)$ the incubation channel that is UV irradiated to crosslink PEGDA, (D) the addition channel to add acidified oil to crosslink alginate, and E) the droplet forming nozzle with inlets for $\mathrm{HRP}$ and $\mathrm{H}_{2} \mathrm{O}_{2}$ to crosslink DexTA. Scale bars depict (C) $500 \mu \mathrm{m}$ or (D,E) $200 \mu \mathrm{m}$.

\section{Microgel formation}

Fluids were infused into chips using syringe pumps (Cetoni $\mathrm{GmbH}$ ). The continuous phase consisted of hexadecane (Sigma-Aldrich) with Span 80 surfactant (1 w/w \%), Sigma-Aldrich), PEGDA, alginate and Dex-TA were used as the dispersed phase. PEGDA (10 w/v \%, $3400 \mathrm{~g} / \mathrm{mol}$, Lysan Bio) in PBS containing 2-hydroxy-4'-(2-hydroxyethoxy)-2-methylpropiophenone (0,2 \%, Irgacure 2959, Sigma-Aldrich) was infused at a flow rate ratio of PEG:Oil 1,4:10 (water/oil ratio $=$ $0,14)$. Droplets were exposed to UV in the delay channel $\left(140 \mathrm{~mW} / \mathrm{cm}^{2}, \mathrm{LC} 8\right.$ Lightningcure L9588, Hamamatsu), unless stated otherwise. This resulted in a gelation time of approximately 8 seconds. Alginate ( $2 \%$, Sigma-Aldrich, low viscosity, 235 CPS) in PBS, supplemented with calcium carbonate nanoparticles $(3,3 \mathrm{mg} / \mathrm{ml}$, Nanomaterials Technology), was used at an alginate:oil flow rate 
ratio of 3:40 (water/oil ratio $=0,075$ ). Alginate was gelated by infusing of acetic acid containing hexadecane $(0,44-1,33 \mu \mathrm{lacid} / \mathrm{ml}$ oil) in the delay channel. DexTA in PBS (10 w/v \%, DS=15, synthesized as described previously ${ }^{[21]}$ ) was co-flown with $\mathrm{HRP}$ and $\mathrm{H}_{2} \mathrm{O}_{2}$ in Dex-TA. The flow rate ratio of Dex-TA:HRP: $\mathrm{H}_{2} \mathrm{O}_{2}$ :oil was respectively 6:1,2:1,2:60 (water/oil ratio $=0,14$ ). Final concentrations were HRP $0,057 \mathrm{mg} / \mathrm{ml}$ (250 units/mg, Sigma-Aldrich) and $\mathrm{H}_{2} \mathrm{O}_{2}$ 15,75 mM (Sigma-Aldrich) unless stated otherwise. Microgels were collected in culture medium, which in the case of alginate was supplemented with $80 \mathrm{mM} \mathrm{CaCl}$ to ensure optimal crosslinking. When encapsulating cells, the hydrogel precursors were laden with $15 * 10^{6} \mathrm{hMSCs}$ per ml.

\section{Scanning Electron Microscopy}

Samples were dehydrated in graded ethanol series (50 \% -100\%), dried from Hexamethyldisilazane (HMDS, Merck), gold sputtered (Cressington) and imaged (XL30 ESEM, FEI).

\section{Viability and metabolic activity}

Live/dead assay was performed using ethidium homodimer-1 and calcein-AM (Invitrogen) according to manufacturer's protocol at 2-4 hours postencapsulation, microphotographed (Nikon E600 fluorescence microscope), and quantified using ImageJ software ( $\mathrm{N}=30$ microgels). Metabolic activity was assessed at day 1, 4, 7, 14, 21 and 28 using the Presto Blue assay (Invitrogen) following manufacturer's protocol. Metabolic activities were normalized to day 1. Presto Blue assay results were corroborated with MTT staining at day 1 and 7 (Sigma-Aldrich) (supplemental figure S1). MTT was added to cell-laden microgels cultures at $0,5 \mathrm{mg} / \mathrm{ml}$, which were microphotographed after two hours of incubation and quantified using ImageJ software ( $N>15$ microgels).

\section{Confocal microscopy}

Gels were fixated using formalin, cytoskeleton was stained with Phalloidin-AF488 (Invitrogen), and nuclei were stained with DRAQ5 (Invitrogen). Stained gels were imaged using confocal microscopy (Nikon A1 confocal microscope). 


\section{Micromechanical testing}

Micromechanical testing was done with a JPK Nanowizard ${ }^{\circledR}$ AFM combined with Zeiss inverted optical microscope, using the Colloidal Probe technique. The cantilever spring constant was $0,151 \mathrm{~N} / \mathrm{m}$ for all samples. Measurements were performed at ambient temperature in PBS, with exception of the alginate samples, which were supplemented with $80 \mathrm{mM}$ of $\mathrm{CaCl}_{2}$ to prevent ion loss.

\section{Statistical analysis}

All experiments consisted of biological triplicates at minimum. All data is shown as average +/- standard deviation (SD), unless stated differently. Data were analysed for statistical significance using ANOVA with Bonferroni post hoc test, or with the Kruskal-Wallis test for non-normal distributed data, with a pvalue of 0,05 .

\section{Results}

\section{Droplet formation and characterization}

Using the universal chip design (Figure 1), we determined the stable droplet formation regime for each polymer. Stable monodispersed droplets were formed in a size range of $137( \pm 2,7)-201( \pm 5,3) \mu \mathrm{m}, 127( \pm 1,9)-142( \pm 6,5) \mu \mathrm{m}$, and $122( \pm 1,6)-168( \pm 8,3) \mu \mathrm{m}$ for PEGDA, Alginate and Dex-TA solutions, respectively (Figure 2 ). In subsequent experiments we used a flow ratio of the dispersed/continuous phase of 0,14 for Dex-TA and PEGDA, resulting in droplets of respectively $157( \pm 3,2)$ and $174( \pm 5,0) \mu \mathrm{m}$ in diameter. At this flow rate, PEGDA containing droplets are exposed to UV-light for approximately 8s. To achieve reasonably stable crosslinking into a microgel within this time frame a minimal UV light intensity of $140 \mathrm{~mW} / \mathrm{cm}^{2}$ was required. Lower UV light intensity or higher flow speed resulted in microgels that disintegrated within a few hours of culture indicative for incomplete crosslinking (data not shown). Higher UV intensity resulted in better crosslinking but this proved even more detrimental for survival of the encapsulated cells as explained in more detail below. The maximum achievable stable flow rate ratio for alginate was noticeably lower $(0,075)$ resulting in droplets of $142( \pm 5,7) \mu \mathrm{m}$. The possible size range of the droplets 
using alginate was restricted, due to the highly viscous nature of the $2 \%$ alginate solution.
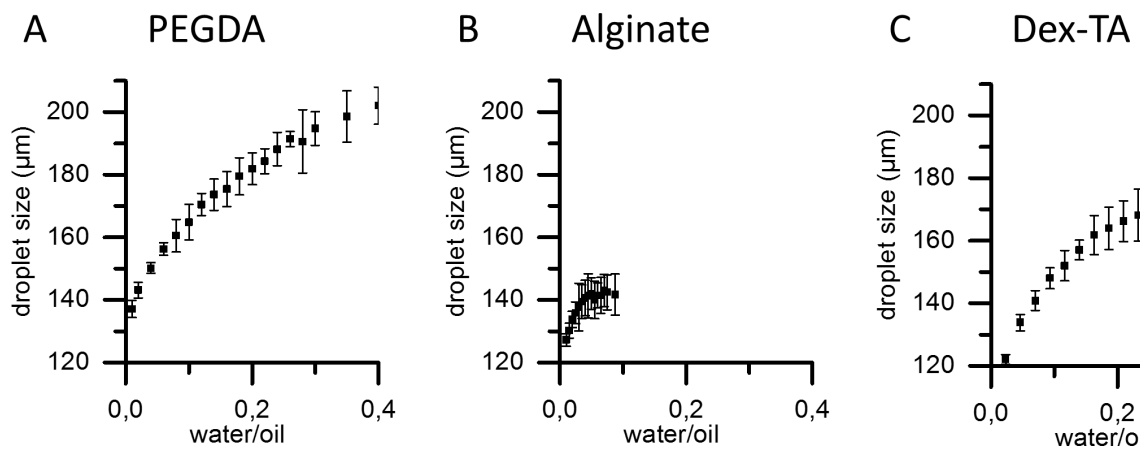

Figure 2. PEGDA, alginate and Dex-TA solutions were infused in the universal chip and droplet sizes were measured on-chip using serial microphotography. Graphs show the average droplet diameter at varying flow speed ratios of (A) PEGDA, (B) alginate and (C) Dex-TA.

\section{Microgel formation}

Droplets of hydrogel precursors with or without cells were produced by emulsification, followed by on-chip crosslinking of the cell-laden gel precursor droplets using UV irradiation, exposure to bivalent $\mathrm{Ca}^{2+}$ ions, or enzymatic reaction. Crosslinked microgels were post-chip collected in culture medium. The emulsion was broken by washing with culture medium and the microgels were collected in the aqueous phase. The fabricated microgels were monodisperse within a narrow size distribution (Figure $3 \mathrm{~A}, \mathrm{~B}, \mathrm{C}$ ). The microgels of Dex-TA were slightly opaque, and have some patches on the surface caused by the rapid initiation of crosslinking (Figure $3 \mathrm{C}$ ). The microgel shape was determined by the channel geometry and the droplet's volume. In particular, microgels were spheres with a flat top and bottom. Scanning Electron Microscopy (SEM) preparation required dehydration of the microgels, which induced microgel shrinkage. SEM analysis confirmed the shape and uniformity of the microgels, particularly of the PEGDA and alginate microgels (Figure $3 \mathrm{D}, \mathrm{E}, \mathrm{F}$ ). The surface of the PEGDA microgels appeared somewhat irregular which is likely an artefact of the dehydration process and incomplete crosslinking (Figure 3D). Alginate gels have some salt residues on them, which precipitated during dehydration. The Dex-TA microgels were smooth, with 2 patches (Figure 3F). These patches likely 
originated from the instantaneous initiation of the crosslinking of the polymer conjugates at the inlets of $\mathrm{HRP}$ and $\mathrm{H}_{2} \mathrm{O}_{2}$ just before droplet formation (Figure 1E).
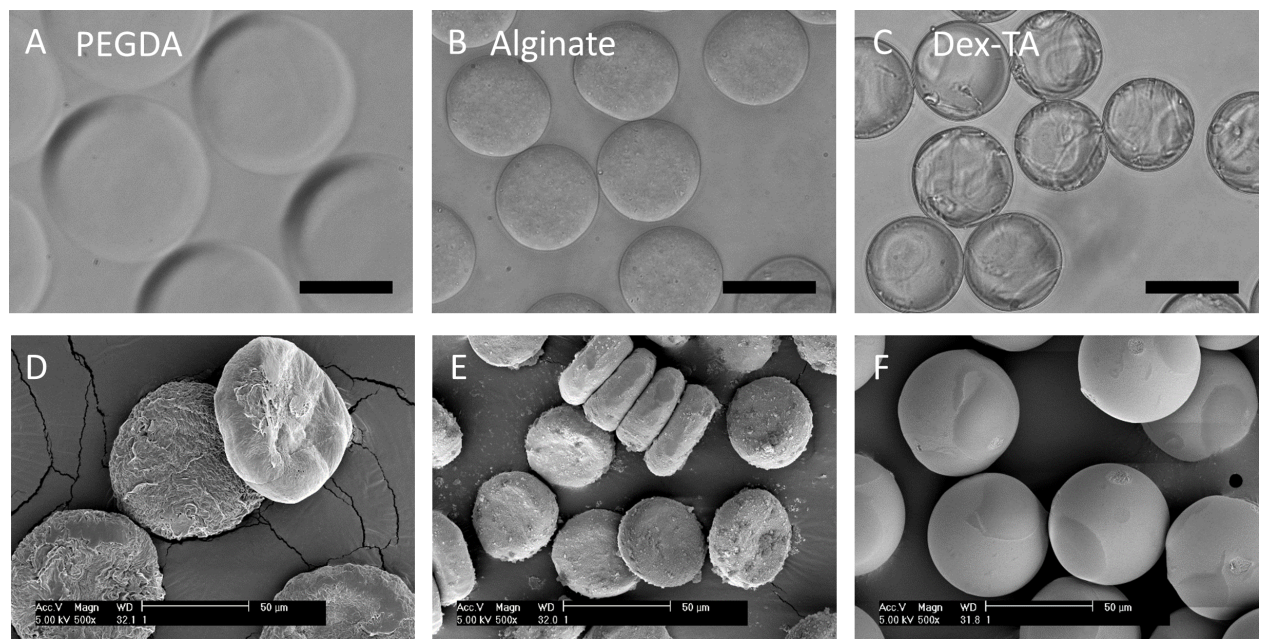

Figure 3. Microgels were microphotographed post-collection using (A-C) brightfield microscopy and (D-F) SEM. Microgels were composed of (A, D) UV crosslinked PEGDA, $(B, E)$ ionically crosslinked alginate, and (C, F) enzymatically crosslinked Dex-TA. Scale bars of (A-C) depict $100 \mu \mathrm{m}$ and (D-F) depict $50 \mu \mathrm{m}$.

\section{Mechanical properties}

The E-modulus of the microgels was measured using atomic force microscopy (Figure 4). UV crosslinked PEGDA microgels proved relatively weak, with an E-modulus of $1,1 \mathrm{kPa}$, which is considerably lower than values reported in literature of a fully cross linked PEGDA hydrogel of similar composition and $\mathrm{wt} / \mathrm{v} \%$, indicative for incomplete crosslinking ${ }^{[22]}$. Moreover, ionically crosslinked alginate microgels were significantly stronger with an E-modulus of to $20,1 \mathrm{kPa}$, and the enzymatically crosslinked Dex-TA microgels demonstrated the highest Emodulus at $30,2 \mathrm{kPa}$. 


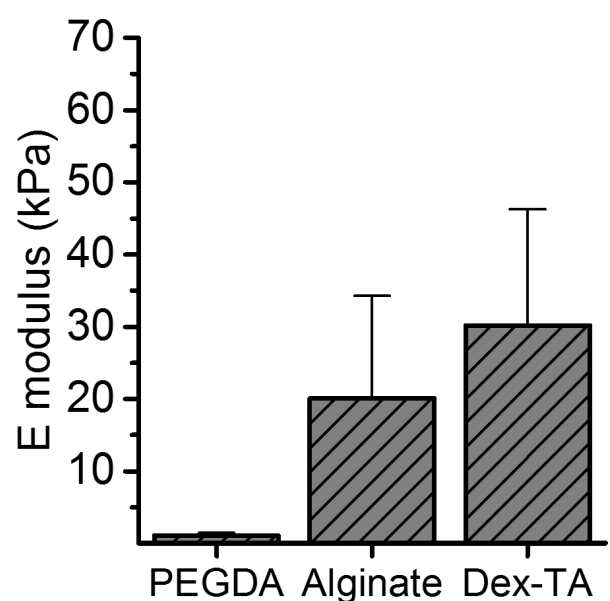

Figure 4. E-moduli of $10 \%$ PEGDA, $2 \%$ alginate and $10 \%$ Dex-TA microgels as measured by AFM.

\section{Cell survival}

We then investigated the survival of hMSCs microencapsulated in PEGDA, alginate and Dex-TA microgels (Figure 5A). For PEGDA microgels, the survival of hMSCs proved inversely correlated with the UV-dose (Figure 5B). Maximal cell survival was $69 \%$ at $140 \mathrm{~mW} / \mathrm{cm}^{2}$ UV irradiation. Usage of lower UV intensity, potentially leading to higher cell survival, was insufficient to crosslink the PEGDA microgels on-chip, as these microgels dissolved within hours of culture (data not shown). For alginate, cell survival correlated with the acetic acid concentration within the oil, which was required to release bivalent $\mathrm{Ca}^{2+}$ ions from the insoluble $\mathrm{CaCO}_{3}$ nanoparticles (Figure $5 \mathrm{~B}$ ). The maximal cell survival for alginate was $81 \%$, which was achieved at an acetic acid concentration of $0,44 \mu \mathrm{l} / \mathrm{ml}$ oil. Lower acetic acid concentrations resulted in the fusion of microgels. In contrast, enzymatic crosslinking resulted in significantly lower cell death, with cell survival rates of $>$ $95 \%$ in a wide range of $\mathrm{H}_{2} \mathrm{O}_{2}$ concentrations (Figure $5 \mathrm{C}$ ). As theoretically every crosslink requires a single $\mathrm{H}_{2} \mathrm{O}_{2}$ molecule, increasing the concentration of $\mathrm{H}_{2} \mathrm{O}_{2}$ increases the total amount of crosslinks. However, a too high concentration of $\mathrm{H}_{2} \mathrm{O}_{2}$ inhibits the function of HRP, thus eventually leading to a reduction in crosslinking rate (data not shown) ${ }^{[23]}$. 


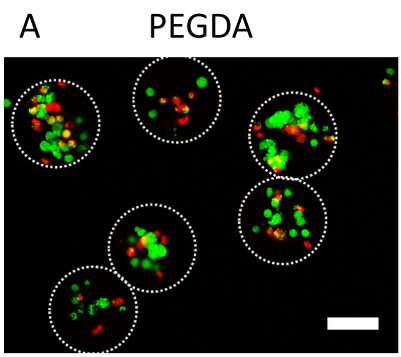

D

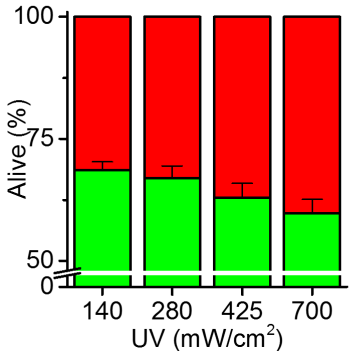

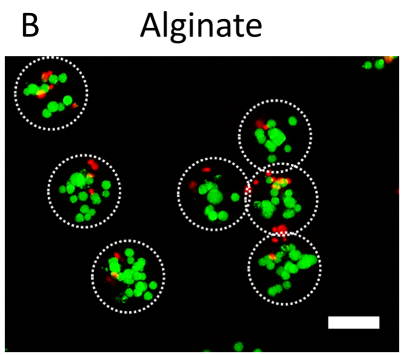

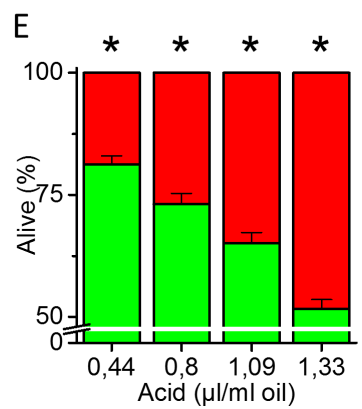

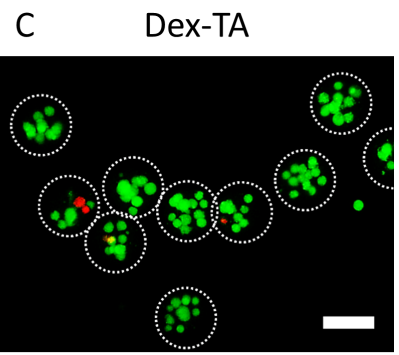

$\mathrm{F}$

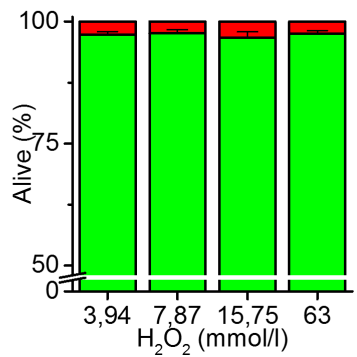

Figure 5. hMSC laden microgels stained for cell survival (green) and cell death (red). (A) PEGDA exposed to $140 \mathrm{~mW} / \mathrm{cm}^{2}$ of UV. (B) Alginate exposed to $0,44 \mu \mathrm{l} / \mathrm{ml}$ of acetic acid. (C) Dex-TA exposed to $15,75 \mathrm{mmol} / \mathrm{l}$ of $\mathrm{H}_{2} \mathrm{O}_{2}$. Scale bars depict $100 \mu \mathrm{m}$. (D,E,F) Semi-quantification cell survival based on live/dead microphotographs of (D) PEGDA, (E) alginate, and (F) Dex-TA. Error bars represent standard error of the mean. * represents a $\mathrm{P}$ value of $<0,05$ compared to the other conditions in the material.

\section{Metabolic activity}

We then microencapsulated hMSCs in Dex-TA, alginate, and PEGDA microgels, and subsequently cultured the microgels for 4 weeks. At predefined regular intervals, the metabolic activity of the encapsulated cells was determined using a presto blue assay. In PEGDA the metabolic activity of hMSCs dropped to undetectable levels within 7 days post encapsulation (Figure 6E). In contrast, hMSCs microencapsulated in alginate remained metabolically active up to at least 7 days (Figure 6F). However, 7 days post-encapsulation the microgels disintegrated, which was likely due to the ion exchange with the culture media. This undermined the ability to reliably measure the encapsulated cells' metabolic activity after day 7. In marked contrast, Dex-TA microgels remained stable over the whole culture period, and the encapsulated cells continued to be metabolically active for at least 28 days. Importantly, after a small initial drop in metabolic activity at day 4 , the metabolic activity progressively increased over 
time (Figure 6G). The metabolic data was corroborated using an MTT assay at day 7 (Figure 6D, Figure S1).
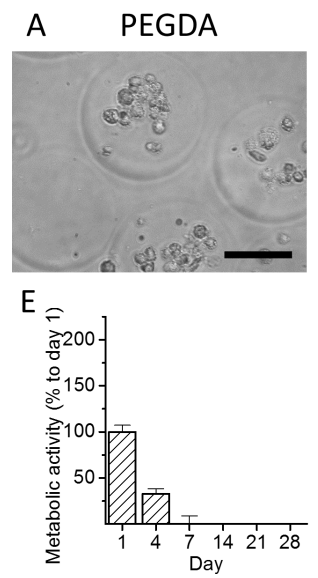
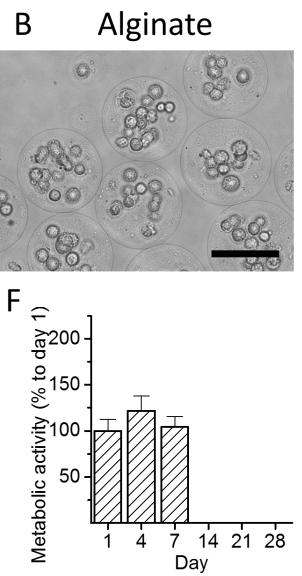
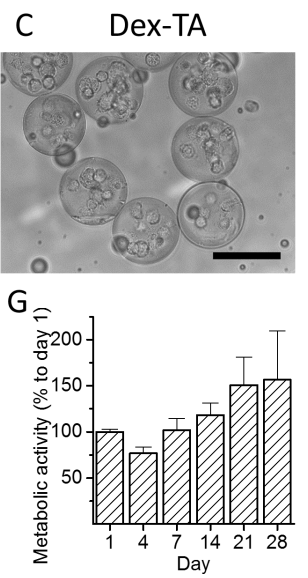
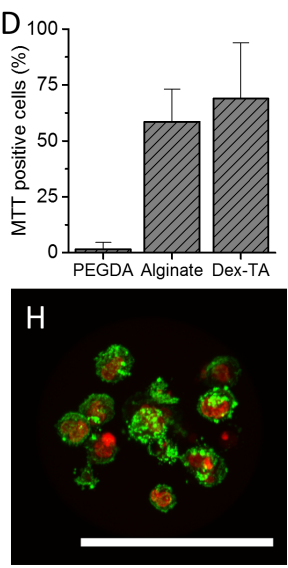

Figure 6. Brightfield microphotographs of hMSCs encapsulated in (A) PEGDA, (B) Alginate, and (C) Dex-TA microgels at day 1 post-crosslinking. (D) MTT Quantification of microgels after 7 days of culture $(n>10)$. Metabolic activity of hMSCs encapsulated in (E) PEGDA, (F) alginate, and (G) DexTA as measured by prestoblue assay on day 1, 4, 714,21 and 28 and normalized to day 1. (H) Zstacked confocal laser scanning microscopy image of the cytoskeleton (green) and nucleus (red) of hMSCs encapsulated in a Dex-TA microgel after 14 days of culture. Scale bar depicts $100 \mu \mathrm{m}$.
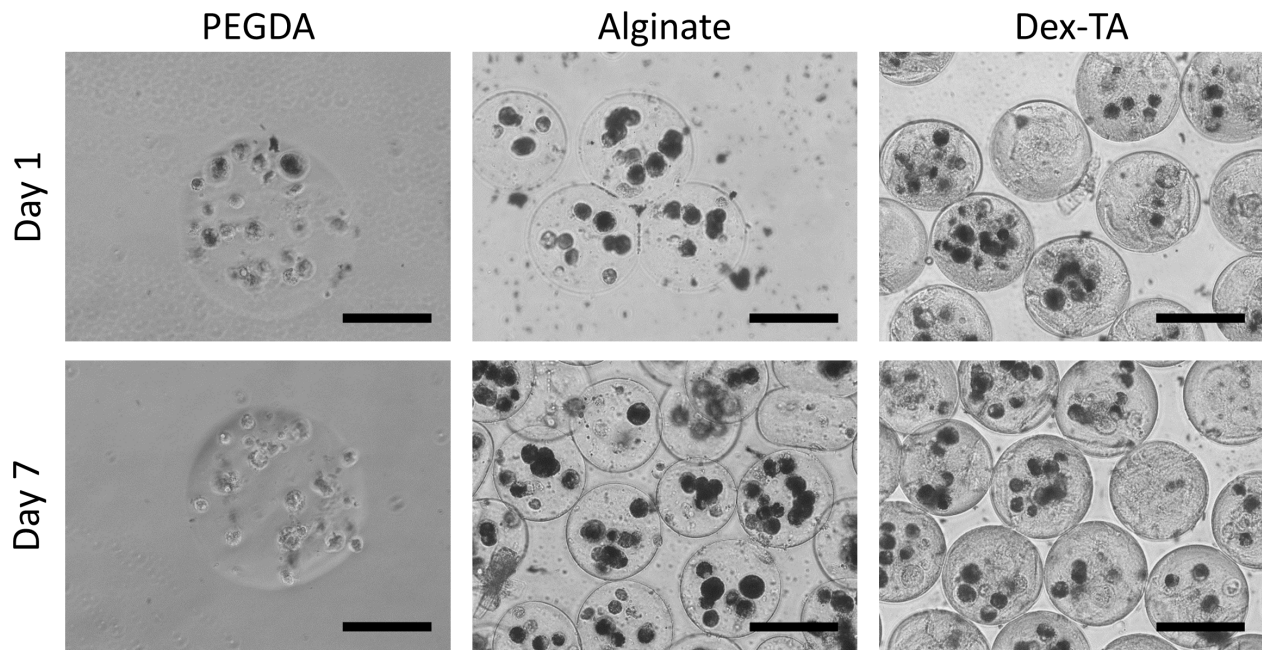

Figure S1. Representative images of MTT stained hMSCs in PEGDA, Alginate and Dex-TA microgels at day 1 and day 7 . Scale bars represent $100 \mu \mathrm{m}$. 


\section{Discussion and Conclusions}

In this paper we have presented a microfluidic platform that was used for on-chip encapsulation of cells in stable microgels in a size range of 120 to $200 \mu \mathrm{m}$. This device allowed for the universal crosslinking of different types of water soluble polymers that are based on an ionic, UV- or enzymatic initiation. Most importantly, we explored enzymatically crosslinking tyramine conjugated polymers as novel materials for microencapsulation, using Dex-TA as a prototype material. Dex-TA macrogels haven been demonstrated to possess excellent properties for cell encapsulation and biofunctionality ${ }^{[17,21,24]}$. Here, we compared enzymatically crosslinked Dex-TA microgels with photo crosslinked PEGDA and ionically crosslinked alginate, using an identical microfluidic droplet generator design.

The on-chip UV based crosslinking of PEGDA demonstrated major drawbacks caused by the limited time window for UV irradiation due to the relatively short on-chip residence time. This therefore demanded high intensity UV exposure to ensure on-chip microgel curing, which caused significant levels of cytotoxicity, as also previously described in literature ${ }^{[25]}$. Surprisingly PEGDA microgels proved to be the mechanically weakest gels in our comparison, and demonstrated limited cell survival, even after minimizing the UV intensity. Although these weak gels could be suited for applications that require a matrix stiffness in the order of magnitude of $1 \mathrm{kPa}$ e.g. for cells of neurogenic lineage, it also limited their ease of handling due to their fragile nature ${ }^{[26]}$. It is expected that further increasing the UV intensity or photoinitiator concentration would produce stronger gels due to a higher density of crosslinks, but would likely also further lower the cell survival. Indeed by increasing the UV intensity from 140 $\mathrm{mW} / \mathrm{cm}^{2}$, which was minimally required to obtain a more or less stable microgel configuration in the $8 \mathrm{~s}$ of retention time in the incubation channel to 700 $\mathrm{mW} / \mathrm{cm}^{2}$, decreased the already low cell survival even further. At the UV intensity of $140 \mathrm{~mW} / \mathrm{cm}^{2}$ crosslinking was still incomplete as demonstrated by rheology. We obtained gels with an E-modulus of approximately $1,1 \mathrm{kPa}$ which is considerably lower compared to the E-modulus of a fully crosslinked $10 \mathrm{wt} / \mathrm{v} \%$ PEGDA hydrogel having an E-modulus larger than $10 \mathrm{kPa}{ }^{[22]}$. Phototoxicity could potentially be mitigated through flow speed and UV reduction, which would also limit the production speed. Alternatively, the microgel's on-chip residence time 
could be enhanced via on-chip delay lines, which would result in suboptimal chip designs, because of high pressure buildup and thus increased chance of chip failure, that still associate with substantial amounts of cell death. In addition, onchip UV based crosslinking of PEGDA microgels induced metabolic cell senescence in the surviving cells within a week, which potentially indicates extensive cell damage. UV induced cell death and damage could also be minimized by crosslinking the microgels off-chip at low intensity for a prolonged period of time [14]. Indeed, photo crosslinking of PEGDA into stable cell laden hydrogels is commonly achieved by crosslinking for at least 5 minutes at relatively low UV intensity [22, 27, 28]. However, this approach would require long retention of droplets off-chip in stable emulsions, which has the adverse effect of demanding additional complex and time consuming procedures. In particular, powerful surfactants will be required for the off chip stabilization of cell encapsulating droplets, which are notoriously difficult to remove. From this work PEGDA appears not very well suited for the on-chip crosslinking polymers into cytocompatible and stable microgels.

Ionic crosslinking of alginate is commonly achieved by dropping alginate in a solution with divalent metal ions such as $\mathrm{Ca}^{2+}$. However, this gelation method, which crosslinks from the outside in, has been found unsuitable for the on-chip fabrication of smaller alginate microgels due to the formation of non-uniform and inhomogeneous microgels ${ }^{[29]}$. Instead, an internal gelation method is required, which is based on solubilizing calcium carbonate particles in the polymer precursor by a temporary decrease in the droplet's $\mathrm{pH}$, using an oil and water soluble acid ${ }^{[29]}$. Unfortunately, this decrease in $\mathrm{pH}$ is toxic to the encapsulated cells. We indeed observed a direct correlation between acid concentration and cell survival, with a maximum survival of $81 \%$, as also found by Tan et al. ${ }^{[29]}$. Furthermore, it is likely that the surviving cells experience substantial amounts of acid induced stress. Another potential disadvantage of this system is the rapid deterioration of the microgels due to progressive loss of calcium ions during culture ${ }^{[30]}$. Deterioration of the microgels progressed on a strikingly higher pace than those reported for macro-sized hydrogels, likely due to the vastly increased surface-to-volume ratio. For short term cultures such as toxicology studies, this fast deterioration is not likely to pose a limitation. However, it does present a challenge for long-term cultures such as cell differentiation, which usually takes 
over 2 weeks. In vitro the microgels can be stabilized by additional supplementations of $\mathrm{Ca}^{2+}$ ions, which might affect cell behavior. Moreover, a repetitive crosslinking approach is not compatible with in vivo applications in a facile manner.

In contrast to these ionic and UV based crosslinking approaches, our body primarily relies on enzymatic induced crosslinking. Examples of enzymes involved in the crosslinking of extracellular matrix molecules are transglutaminase, lysyl oxidase, and horseradish peroxidase ${ }^{[31-33]}$. A major advantage of this approach is its ability to crosslink at physiological conditions, without requiring harsh chemicals. In this study, we explored microgel formation using the enzymatic crosslinking of Dex-TA, which was achieved by on-chip mixing of polymer precursor with the enzyme HRP and $\mathrm{H}_{2} \mathrm{O}_{2}$. In contrast to UV or acid induced systems, enzymatic crosslinking strategies are well-known for their cytocompatibility. Although it might be intuitive to reason that the use of $\mathrm{H}_{2} \mathrm{O}_{2}$ might induce cytotoxic events, the required levels of $\mathrm{H}_{2} \mathrm{O}_{2}$ are far below those that induce cell damage or death, as the minute quantities of $\mathrm{H}_{2} \mathrm{O}_{2}$ are rapidly converted during the enzymatic reaction. Indeed, cell survival after microgel formation was substantially higher as compared to other reported microgel crosslinking strategies, reaching levels as high as $95 \%$. In fact, the observed level of cell death was identical to those generated by routine cell passaging. The cellfriendliness of this approach was further underlined by the observation that the cell survival was not adversely affected by increasing the $\mathrm{H}_{2} \mathrm{O}_{2}$ concentrations to levels that exceeded the functional crosslinking concentration. Dex-TA microgels of $10 \%$ Dextran were with $30 \mathrm{kPa}$ mechanically very strong. This makes Dex-TA microgels very suitable for differentiation towards the musculoskeletal lineage, were cartilage needs $20-30 \mathrm{kPa}$ and bone $25-40 \mathrm{kPa}{ }^{[26,34]}$. However, other tissues like nerve or brain $(0,1-1 \mathrm{kPa})$ and fat $(3 \mathrm{kPa})$ require softer gels ${ }^{[26,34]}$. In order to match these, the mechanical properties can be tuned by varying the wt $/ \mathrm{v} \%$ of the gel and the degree of substitution of the dextran ${ }^{[17,21]}$.

Encapsulating cells in micromaterials that in turn will be encapsulated in a macro-sized scaffold opens up numerous potential avenues in creating multiscale bioengineered constructs. In particular, it is conducive for the decoupling of the biomaterial that is proximal to the cells and the bulk biomaterial that is more distal to cells ${ }^{[35]}$. This strategy resembles the natural organization of 
cells in tissue, in which cells are surrounded by a pericellular matrix, which together is embedded in an extracellular matrix. The composition and thus function differs greatly between these matrices. Our strategy provides a roadmap towards engineering such complex and biologically relevant organizations. This approach holds great potential to advance tissue engineering by adding biological complexity in a facile manner to the tissue engineered constructs ${ }^{[36-38]}$. This development could additionally contribute to other fields like (stem)cell biology, drug development and pathophysiology, by driving the development of more biomimetic ex vivo models ${ }^{[39-41]}$. Moreover, gaining the capability to create cellfriendly customizable cellular microniches provides possibilities for efficient high throughput screens with single-cell resolution ${ }^{[42]}$. This might be of particular interest as microgels can yield information on the single cell level by confocal microscopic analysis of the entire gel, and allow for facile downstream analysis ${ }^{[42]}$. This approach allows for the precise probing of cellular heterogeneity to a given biophysical, chemical or environmental stimuli to identify of e.g. differently responsive sub-populations of cells. Moreover, these 3D biomimetic microtissues can act as smart building blocks to create complex functional tissues following a bottom up tissue engineering approach ${ }^{[43]}$.

However, to create truly biomimetic microgels, the incorporation of biomimetic elements such as extracellular matrix components, growth factor binding sites, and cell responsive elements such as catabolically cleavable moieties will be of deciding importance. Advantageously, enzymatically crosslinked biomaterials such as Dex-TA allow for straightforward decoration with such elements. For example, cell instructing and growth factor capturing matrix molecules including hyaluronic acid, heparin, and chondroitin sulfate can be engrafted on the polymer's backbone or co-crosslinked using e.g. tyramine modified molecules ${ }^{[18,19,44]}$. Enzymatic crosslink strategies such as used for DexTA can also covalently bond a myriad of molecules including collagens by crosslinking with their tyrosine residues ${ }^{[45]}$.

In summary, enzymatically crosslinked hydrogels are ideal for the generation of microfluidically generated microgels. This platform is expected to contribute to the future development of custom-designed 3D biomimetic microtissues using a plethora of existing, simple and cost-effective methods to enable cell-based screenings with a single cell resolution. 


\section{Acknowledgements}

The authors gratefully thank Johan Bomer of the BIOS Lab-on-a-chip group of University of Twente for his expertise in micromolding, Tom Kamperman and Jan Hendriks of the Developmental BioEngineering group of University of Twente for their assistance with Matlab analysis.

\section{References}

[1] B. Ma, J. C. Leijten, L. Wu, M. Kip, C. A. van Blitterswijk, J. N. Post, and M. Karperien, "Gene expression profiling of dedifferentiated human articular chondrocytes in monolayer culture", Osteoarthritis and Cartilage, vol. 21, pp. 599-603, 2013.

[2] P. Godoy, J. G. Hengstler, I. Ilkavets, C. Meyer, A. Bachmann, A. Müller, G. Tuschl, S. O. Mueller, and S. Dooley, "Extracellular matrix modulates sensitivity of hepatocytes to fibroblastoid dedifferentiation and transforming growth factor $\beta$-induced apoptosis", Hepatology, vol. 49, pp. 2031-2043, 2009.

[3] D.-A. Lacorre, E. S. Baekkevold, I. Garrido, P. Brandtzaeg, G. Haraldsen, F. Amalric, and J.-P. Girard, "Plasticity of endothelial cells: rapid dedifferentiation of freshly isolated high endothelial venule endothelial cells outside the lymphoid tissue microenvironment", Blood, vol. 103, pp. 4164-4172, 2004.

[4] J. Leijten, Y. C. Chai, I. Papantoniou, L. Geris, J. Schrooten, and F. P. Luyten, "Cell based advanced therapeutic medicinal products for bone repair: Keep it simple?", Adv Drug Deliv Rev, vol. 84, pp. 30-44, 2015.

[5] J. Leijten, J. Rouwkema, Y. S. Zhang, A. Nasajpour, M. R. Dokmeci, and A. Khademhosseini, "Advancing Tissue Engineering: A Tale of Nano-, Micro, and Macroscale Integration", Small, vol. 12, pp. 2130-45, 2016.

[6] J. Lee, M. J. Cuddihy, and N. A. Kotov, "Three-dimensional cell culture matrices: State of the art", Tissue Engineering Part B-Reviews, vol. 14, pp. 61-86, 2008.

[7] A. Kang, J. Park, J. Ju, G. S. Jeong, and S.-H. Lee, "Cell encapsulation via microtechnologies", Biomaterials, vol. 35, pp. 2651-2663, 2014.

[8] P. de Vos, H. A. Lazarjani, D. Poncelet, and M. M. Faas, "Polymers in cell encapsulation from an enveloped cell perspective", Adv Drug Deliv Rev, vol. 67-68, pp. 15-34, 2014.

[9] S. Allazetta, L. Kolb, S. Zerbib, J. a. Bardy, and M. P. Lutolf, "Cell-Instructive Microgels with Tailor-Made Physicochemical Properties", Small, vol. 11, pp. 5647-5656, 2015. 
[10] C. J. Martinez, J. W. Kim, C. Ye, I. Ortiz, A. C. Rowat, M. Marquez, and D. Weitz, "A Microfluidic Approach to Encapsulate Living Cells in Uniform Alginate Hydrogel Microparticles", Macromolecular Bioscience, vol. 12, pp. 946-951, 2012.

[11] E. Tumarkin and E. Kumacheva, "Microfluidic generation of microgels from synthetic and natural polymers", Chemical Society Reviews, vol. 38, pp. 2161-8, 2009.

[12] E. Santos, J. Zarate, G. Orive, R. M. Hernandez, and J. L. Pedraz, "Biomaterials in cell microencapsulation", Advances in Experimental Medicine and Biology, vol. 670, pp. 5-21, 2010.

[13] Ý. A. Mørch, I. Donati, and B. L. Strand, "Effect of Ca2+, Ba2+, and Sr2+ on Alginate Microbeads", Biomacromolecules, vol. 7, pp. 1471-1480, 2006.

[14] Y. Ma, M. P. Neubauer, J. Thiele, A. Fery, and W. T. S. Huck, "Artificial microniches for probing mesenchymal stem cell fate in 3D", Biomater. Sci., vol. 2, pp. 1661-1671, 2014.

[15] C. Kielbassa, L. Roza, and B. Epe, "Wavelength dependence of oxidative DNA damage induced by UV and visible light", Carcinogenesis, vol. 18, pp. 811-6, 1997.

[16] J. Clausell-Tormos, D. Lieber, J.-C. Baret, A. El-Harrak, O. J. Miller, L. Frenz, J. Blouwolff, K. J. Humphry, S. Köster, H. Duan, C. Holtze, D. A. Weitz, A. D. Griffiths, and C. A. Merten, "Droplet-Based Microfluidic Platforms for the Encapsulation and Screening of Mammalian Cells and Multicellular Organisms", Chemistry \& Biology, vol. 15, pp. 427-437, 2008.

[17] R. Jin, C. Hiemstra, Z. Zhong, and J. Feijen, "Enzyme-mediated fast in situ formation of hydrogels from dextran-tyramine conjugates", Biomaterials, vol. 28, pp. 2791-800, 2007.

[18] J. W. H. Wennink, K. Niederer, A. I. Bochyńska, L. S. Moreira Teixeira, M. Karperien, J. Feijen, and P. J. Dijkstra, "Injectable Hydrogels by Enzymatic Co-Crosslinking of Dextran and Hyaluronic Acid Tyramine Conjugates", Macromolecular Symposia, vol. 309-310, pp. 213-221, 2011.

[19] R. Jin, L. S. Moreira Teixeira, P. J. Dijkstra, C. A. van Blitterswijk, M. Karperien, and J. Feijen, "Chondrogenesis in injectable enzymatically crosslinked heparin/dextran hydrogels", Journal of Controlled Release, vol. 152, pp. 186-195, 2011.

[20] S. K. Both, A. J. van der Muijsenberg, C. A. van Blitterswijk, J. de Boer, and J. D. de Bruijn, "A rapid and efficient method for expansion of human mesenchymal stem cells", Tissue Engineering, vol. 13, pp. 3-9, 2007.

[21] R. Jin, L. S. Moreira Teixeira, P. J. Dijkstra, Z. Zhong, C. A. van Blitterswijk, M. Karperien, and J. Feijen, "Enzymatically crosslinked dextran-tyramine hydrogels as injectable scaffolds for cartilage tissue engineering", Tissue engineering. Part $A$, vol. 16, pp. 2429-40, 2010. 
[22] S. J. Bryant and K. S. Anseth, "Hydrogel properties influence ECM production by chondrocytes photoencapsulated in poly(ethylene glycol) hydrogels", Journal of Biomedical Materials Research, vol. 59, pp. 63-72, 2002.

[23] J. A. Nicell and H. Wright, "A model of peroxidase activity with inhibition by hydrogen peroxide", Enzyme and Microbial Technology, vol. 21, pp. 302-310, 1997.

[24] L. S. Moreira Teixeira, J. C. Leijten, J. Sobral, R. Jin, A. A. van Apeldoorn, J. Feijen, C. van Blitterswijk, P. J. Dijkstra, and M. Karperien, "High throughput generated micro-aggregates of chondrocytes stimulate cartilage formation in vitro and in vivo", Eur Cell Mater, vol. 23, pp. 38799, 2012.

[25] P. Panda, S. Ali, E. Lo, B. G. Chung, T. A. Hatton, A. Khademhosseini, and P. S. Doyle, "Stop-flow lithography to generate cell-laden microgel particles", Lab on a Chip, vol. 8, p. 1056, 2008.

[26] A. J. Engler, S. Sen, H. L. Sweeney, and D. E. Discher, "Matrix Elasticity Directs Stem Cell Lineage Specification", Cell, vol. 126, pp. 677-689, 2006.

[27] C. G. Williams, T. K. Kim, A. Taboas, A. Malik, P. Manson, and J. Elisseeff, "In vitro chondrogenesis of bone marrow-derived mesenchymal stem cells in a photopolymerizing hydrogel", Tissue Engineering, vol. 9, pp. 679-88, 2003.

[28] G. Musumeci, C. Loreto, M. L. Carnazza, I. Strehin, and J. Elisseeff, "OA cartilage derived chondrocytes encapsulated in poly(ethylene glycol) diacrylate (PEGDA) for the evaluation of cartilage restoration and apoptosis in an in vitro model", Histology and Histopathology, vol. 26, pp. 1265-78, 2011.

[29] W. H. Tan and S. Takeuchi, "Monodisperse Alginate Hydrogel Microbeads for Cell Encapsulation", Advanced Materials, vol. 19, pp. 2696-2701, 2007.

[30] M. Moya, M. Morley, O. Khanna, E. Opara, and E. Brey, "Stability of alginate microbead properties in vitro", Journal of Materials Science: Materials in Medicine, vol. 23, pp. 903-912, 2012.

[31] L. I. Smith-Mungo and H. M. Kagan, "Lysyl oxidase: Properties, regulation and multiple functions in biology", Matrix Biology, vol. 16, pp. 387-398, 1998.

[32] D. Aeschlimann, O. Kaupp, and M. Paulsson, "Transglutaminase-catalyzed matrix cross-linking in differentiating cartilage: Identification of osteonectin as a major glutaminyl substrate", Journal of Cell Biology, vol. 129, pp. 881-892, 1995. 
[33] F. Passardi, C. Penel, and C. Dunand, "Performing the paradoxical: how plant peroxidases modify the cell wall", Trends in Plant Science, vol. 9, pp. 534-40, 2004.

[34] A. Buxboim, I. L. Ivanovska, and D. E. Discher, "Matrix elasticity, cytoskeletal forces and physics of the nucleus: how deeply do cells 'feel' outside and in?", Journal of Cell Science, vol. 123, pp. 297-308, 2010.

[35] S. Guven, P. Chen, F. Inci, S. Tasoglu, B. Erkmen, and U. Demirci, "Multiscale assembly for tissue engineering and regenerative medicine", Trends in Biotechnology, vol. 33, pp. 269-279, 2015.

[36] Y. Du, E. Lo, S. Ali, and A. Khademhosseini, "Directed assembly of cellladen microgels for fabrication of 3D tissue constructs", Proceedings of the National Academy of Sciences, vol. 105, pp. 9522-9527, 2008.

[37] Y. T. Matsunaga, Y. Morimoto, and S. Takeuchi, "Molding Cell Beads for Rapid Construction of Macroscopic 3D Tissue Architecture", Advanced Materials, vol. 23, pp. H90-H94, 2011.

[38] J. Leijten and A. Khademhosseini, "From Nano to Macro: Multiscale Materials for Improved Stem Cell Culturing and Analysis", Cell Stem Cell, vol. 18, pp. 20-4, 2016.

[39] D. E. Discher, D. J. Mooney, and P. W. Zandstra, "Growth Factors, Matrices, and Forces Combine and Control Stem Cells", Science, vol. 324, pp. 1673-1677, 2009.

[40] M.-Y. Lee, R. A. Kumar, S. M. Sukumaran, M. G. Hogg, D. S. Clark, and J. S. Dordick, "Three-dimensional cellular microarray for high-throughput toxicology assays", Proceedings of the National Academy of Sciences, vol. 105, pp. 59-63, 2008.

[41] H. Onoe, T. Okitsu, A. Itou, M. Kato-Negishi, R. Gojo, D. Kiriya, K. Sato, S. Miura, S. Iwanaga, K. Kuribayashi-Shigetomi, Y. T. Matsunaga, Y. Shimoyama, and S. Takeuchi, "Metre-long cell-laden microfibres exhibit tissue morphologies and functions", Nat Mater, vol. 12, pp. 584-90, 2013.

[42] S. Gobaa, S. Hoehnel, M. Roccio, A. Negro, S. Kobel, and M. P. Lutolf, "Artificial niche microarrays for probing single stem cell fate in high throughput", Nature Methods, vol. 8, pp. 949-55, 2011.

[43] A. Khademhosseini and R. Langer, "Microengineered hydrogels for tissue engineering", Biomaterials, vol. 28, pp. 5087-5092, 2007.

[44] L. S. Moreira Teixeira, J. C. H. Leijten, J. W. H. Wennink, A. G. Chatterjea, J. Feijen, C. A. van Blitterswijk, P. J. Dijkstra, and M. Karperien, "The effect of platelet lysate supplementation of a dextran-based hydrogel on cartilage formation", Biomaterials, vol. 33, pp. 3651-3661, 2012.

[45] L. S. Moreira Teixeira, S. Bijl, V. V. Pully, C. Otto, R. Jin, J. Feijen, C. A. van Blitterswijk, P. J. Dijkstra, and M. Karperien, "Self-attaching and cell- 
attracting in-situ forming dextran-tyramine conjugates hydrogels for arthroscopic cartilage repair", Biomaterials, vol. 33, pp. 3164-3174, 2012. 


\section{Chapter 3}

The stiffness and composition of micro hydrogels independently control mesenchymal stromal cell differentiation in the osteogenic lineage

Sieger Henke, Tom Kamperman, Rong Wang, Pieter J Dijkstra, Jeroen Leijten, Marcel Karperien 


\section{Abstract}

Mesenchymal stromal cells (MSCs) are multipotent progenitor cells that can differentiate towards amongst others the osteogenic and adipogenic lineage. This makes them useful for tissue engineering of both bone and soft tissue. Here we investigated a strategy in which the cells are encapsulated in microgels made of enzymatically crosslinked tyramine conjugated polymers. Soft gels of dextrantyramine or a combination of dextran-tyramine and hyaluronic acid-tyramine with an E-modulus of 3-3,5 kPa favored adipogenic differentiation. Stiff dextrantyramine gels of $21 \mathrm{kPa}$ favored osteogenic differentiation. Remarkably, differentiation towards the osteogenic lineage was inhibited by the addition of hyaluronic acid-tyramine, both in stiff and in soft gels. This suggests that matrix composition and stiffness independently control MSC differentiation in the osteogenic lineage. The underlying mechanisms for these findings are unknown and warrant further research. Our platform enables the creation of specialized microenvironments for the bottom-up tissue engineering of functional constructs for bone and soft tissue reconstruction. 


\section{Introduction}

Tissue engineering using cells and hydrogels holds great promise for amongst others the repair of critical size bone and cartilage defects, and to fill soft tissue defects. However, in its most simplified top-down strategy, filling the defect with cells or cells in hydrogels, has not been very successful in the clinic ${ }^{[1]}$. This might be due to homogenous nature of such constructs which is in marked contrast to the complexity of native tissue. To recreate this natural complexity in large tissue engineered structures, the concept of bottom-up tissue engineering has been introduced ${ }^{[1]}$. Bottom-up tissue engineering uses small building blocks to assemble a larger construct ${ }^{[2]}$. One of the cornerstones of bottom-up tissue engineering are cell-laden (micro)hydrogels that can be produced in a variety of shapes using microfabrication technology. Examples of microfabrication technologies used for engineering building blocks for bottom-up tissue engineering are droplet microfluidics ${ }^{[3]}$ and lithography techniques ${ }^{[4]}$. For example, Matsunaga et al. ${ }^{[3]}$ created molds controlling the shape of the assembly of cell laden hydrogels. Du et al. ${ }^{[4]}$ made microgel units out of hydrogel precursors that were crosslinked into stable 3D gels by photo crosslinking through a photomask. These relatively small shapes in the micrometer range could subsequently self-assemble in larger constructs in the centimeter range.

A variety of cell sources can be used in bottom-up tissue engineering approaches. Adult Mesenchymal Stromal Cells (MSCs) are one of the most frequently used cell sources. MSCs can be easily isolated from amongst other the bone marrow. These cells can be easily expanded in vitro to obtain sufficient cells for a particular purpose and are well known for their multi lineage differentiation capacity into amongst others bone forming osteoblasts and fat depositing adipocytes ${ }^{[5]}$.

Multiple factors determine the differentiation of the MSCs into a particular lineage ${ }^{[6]}$. The use of cocktails of specific growth factors or other medium additives has been best studied. However, differentiation control and subsequent function of the cells is also highly dependent on environmental cues such as provided by cell culture substrates. Both the stiffness and the composition of these substrates are important parameters involved in differentiation control ${ }^{[7-9]}$. The cells can sense the stiffness of the matrix by physical interactions predominantly mediated via integrins that bind to RGD-sequences present in 
many extracellular matrix proteins. Pulling at the matrix by the cell results in activation of mechanotransduction signaling pathways whose signals are based on the force exerted by the cell that is needed to deform the matrix ${ }^{[10]}$. Matrices with low stiffness, approximately below $4 \mathrm{kPa}$, favor adipogenic differentiation ${ }^{[6,}$ 9, 11], where matrices with higher stiffness, approximately over $10 \mathrm{kPa}$, favor osteogenic differentiation of MSCs ${ }^{[9,10,12]}$.

Next to stiffness, the composition of the matrix in which the cells are embedded is also important. Hydrogels are an ideal matrix to embed and differentiate cells in, as they can resemble many characteristics of the native extracellular matrix. Numerous types of hydrogels are pioneered to encapsulate cells. Alginate was one of the first materials used for cell encapsulation. It is a natural polymer extracted from algae, and crosslinked using divalent ions, usually $\mathrm{Ca}^{2+}$, in a macromolecular hydrogel network. Poly ethylene glycol (PEG) is another frequently applied material for cell encapsulation. PEG is a synthetic polymer often functionalized with acrylate groups rendering a material that can crosslink in a macromolecular network using photo polymerization. Both materials have disadvantages, such as for alginate the instability of the network on the long run due to leaching of ions in ionically crosslinked gels and for PEG the toxicity of the UV-mediated photo crosslinking.

An upcoming category of materials for cell encapsulation in hydrogels are enzymatically crosslinked polymers. An example is the crosslinking of conjugates of polysaccharides with tyramine in stable networks upon mixing with the enzyme horseradish peroxidase and non-toxic concentrations of hydrogen peroxide as initiator ${ }^{[13]}$. Enzymatic crosslinking usually occurs under physiological conditions and is highly biocompatible. Examples of relevant polysaccharides conjugates that have been used for generating injectable hydrogels are bio-inert conjugates of dextran with tyramine, and the body's own hyaluronic acid with tyramine.

We have previously developed a platform for the on-chip production of enzymatically crosslinked cell-laden micro hydrogels using dextran-tyramine conjugates ${ }^{[14]}$. The on-chip encapsulation of cells in dextran-based macromolecular networks was cell friendly with neglectable (less then few \%) cell death. Furthermore, the microgels were stable and cells could be cultured inside these microgels for weeks without compromising cell function. Uniquely, with 
this system we can produce multi-cell-laden microgels and use these constructs as building blocks in bottom up tissue engineering. In such approach multi-cell microgels have several advantages over single cell microgels as presented by Ma and coworkers ${ }^{[15]}$. The cell density can be higher, which can be important for cell survival and differentiation ${ }^{[16,17]}$, and the cell throughput in microgels production is higher due to higher cell concentrations, which is favorable when high cell numbers are needed.

In this work we have used this method to fabricate MSC-laden multi-cell microgels composed of either dextran-tyramine conjugates (Dex) or of a 50:50 mixture of dextran-tyramine and hyaluronic acid-tyramine conjugates (DexHA) at a weight volume (wt/v) percentage of $5 \%$ and $10 \%$. This allowed us to study the effect of matrix stiffness and matrix composition on MSC differentiation into the adipogenic and osteogenic lineage

\section{Materials and Methods}

\section{Hydrogel material}

Dextran-tyramine (MW $18460 \mathrm{~g} / \mathrm{mol}$ (15-30 kg/mol, Sigma-Aldrich)) and hyaluronic acid-tyramine (MW $16000 \mathrm{~g} / \mathrm{mol}$ (15-30 kg/mol, Contipro)) conjugates were prepared as described previously ${ }^{[18]}$. Dex-TA was used with a degree of substitution (DS, number of tyramine residues per 100 repeating monosaccharide blocks) of 15, HA-TA with a DS of $2.5 \mathrm{wt} / \mathrm{v} \%$ gels contained $14,3 \mathrm{U} / \mathrm{ml} \mathrm{HRP}$ (horseradish peroxidase) and $0,008 \mathrm{mmol} / \mathrm{ml} \mathrm{H}_{2} \mathrm{O}_{2}, 10 \mathrm{wt} / \mathrm{v} \%$ gels contained 28,6 $\mathrm{U} / \mathrm{ml} \mathrm{HRP}$ and $0,016 \mathrm{mmol} / \mathrm{ml} \mathrm{H}_{2} \mathrm{O}_{2}$.

\section{Rheology}

$250 \mu \mathrm{l}$ gels were prepared on ice and moved to room temperature to start the gelation. After gelation the gels were transferred to a rheometer (Anton Paar, Oosterhout, The Netherlands), in between two flat plates. The gels were measured with a $250 \mu \mathrm{m}$ gap size, and a frequency of $0,5 \mathrm{~Hz}$ and $0,1 \%$ strain.

$5 \%$ gels contained $14,3 \mathrm{U} / \mathrm{ml} \mathrm{HRP}$ and $0,008 \mathrm{mmol} / \mathrm{ml} \mathrm{H}_{2} \mathrm{O}_{2}, 10 \%$ Gels contained $28,6 \mathrm{U} / \mathrm{ml} \mathrm{HRP}$ and $0,016 \mathrm{mmol} / \mathrm{ml} \mathrm{H}_{2} \mathrm{O}_{2}$, to retain a constant ratio between the number of tyramine residues and $\mathrm{HRP}$ and $\mathrm{H}_{2} \mathrm{O}_{2}$.

The $E$ modulus was calculated from the storage modulus $G$, assuming a linear elastic material and Poisson's ratio $v=0,5$ as follows: 


$$
E=2 G^{\prime}(1+v)
$$

\section{Microfluidic chip preparation}

Microgels were formed using a microfluidic device. This device was prepared using soft lithography techniques as described previously ${ }^{[14]}$. In short: the chip was designed using CAD software, subsequently the design was transferred to a photomask. A $70 \mu \mathrm{m}$ layer of SU-8 polymer was applied on a silicon wafer and photolithography was performed using the photomask. Subsequently, the wafer was used to prepare a PDMS replicate (Sylgard 184, DowCorning). For this, PDMS was mixed in a ratio of 10:1 base:curing agent, applied on the wafer and allowed to cure. The PDMS was removed from the wafer, cut to size, and holes were punched for inlets and outlet. Finally, the PDMS replicate was bound to a glass slide using oxygen plasma.

\section{Microgel formation}

Microgels were formed as described previously (chapter 4 of this thesis) [19]. Fluids were infused using syringe pumps. The dispersed aqueous phase consisted of 3 components: the polymer solution containing the cells and $8 \%$ optiprep (Sigma-Aldrich), the polymer solution containing HRP and a polymer solution containing a small amount of hydrogen peroxide. Directly after joining of the three components droplets of approximately $100 \mu \mathrm{m}$ were formed in a flow focusing geometry using hexadecane (Sigma-Aldrich) with 1 \% Span 80 (SigmaAldich) as the continuous phase. A $140 \mathrm{~mm}$ delay channel was used for gelation, and the gelated droplets, now microgels, were collected via tubing in culture medium. Cells were encapsulated at a density of $10 * 10^{6}$ cells $/ \mathrm{ml}$. 4 types of gels were produced: $5 \mathrm{wt} / \mathrm{v} \%$ Dex-TA (5\% Dex), $5 \mathrm{wt} / \mathrm{v} \%$ Dex-TA/HA-TA (50:50\%, 5\% DexHA), $10 \mathrm{wt} / \mathrm{v} \%$ Dex-TA (10\% Dex) and $10 \mathrm{wt} / \mathrm{v} \%$ Dex-TA/HA-TA (50:50\%, 10\% DexHA),

\section{Cell viability}

The cell viability was assessed with the LIVE/DEAD kit (Invitrogen) according to manufacturer's protocol. Images were taken on an EVOS digital microscope (Westburg/Invitrogen). 


\section{Microscopy}

Brightfield and phase contrast images were taken on a Nikon Eclipse TE300 microscope equipped with Nikon Digital Sight DS-fi1 camera.

\section{Cell culture}

The use of patient material was approved by the local ethical committee of the Medisch Spectrum Twente, and informed written consent was obtained for all samples. Human mesenchymal stem cells (hMSCs) were isolated from bone marrow aspirates as described previously ${ }^{[20]}$. The cells were cultured in alfa-MEM (Invitrogen), supplemented with 10 \% FBS (Sigma-Aldrich), 2 mM GlutaMAX (Invitrogen), 0,2 $\mathrm{mM}$ ascorbic acid (Sigma-Aldrich), $1 \mathrm{ng} / \mathrm{ml}$ basic fibroblast growth factor (ISOKine bFGF, Neuromics), and $100 \mathrm{U} / \mathrm{ml}$ penicillin and $100 \mu \mathrm{g} / \mathrm{ml}$ streptomycin (Invitrogen). Cells were kept in a humidified environment with $5 \%$ $\mathrm{CO} 2$ and used from passage 3 to 5 .

\section{Differentiation}

Differentiation was started the day after encapsulation in the microgels and the day after seeding of the monolayer. Each well of a 24-well plate contained $8 \mu \mathrm{l}$ of gel with 80000 cells encapsulated and 1,5 $\mathrm{ml}$ of culture medium. Monolayer differentiation was seeded at 10000 cells per $\mathrm{cm}^{2}$ for adipogenic differentiation and 1000 cells per $\mathrm{cm}^{2}$ for osteogenic differentiation.

The medium composition for osteogenic differentiation was Alfa-MEM (Invitrogen), supplemented with 10\% FBS (Sigma-Aldrich), $100 \mathrm{U} / \mathrm{ml}$ penicillin and $100 \mathrm{\mu g} / \mathrm{ml}$ streptomycin (Invitrogen), $2 \mathrm{mM}$ GlutaMAX (Invitrogen), 0,2 mM ascorbic acid (Sigma-Aldrich), 0,01 $\mu \mathrm{M}$ Dexamethasone $\left(10^{-8} \mathrm{M}\right.$, Sigma-Aldrich) and 0,01 M beta-glycerol phosphate (Sigma-Aldrich).

The medium composition for adipogenic differentiation consisted of DMEM (Invitrogen), supplemented with 10 \% FBS (Sigma-Aldrich), 100 U/ml penicillin and $100 \mu \mathrm{g} / \mathrm{ml}$ streptomycin (Invitrogen), $2 \mathrm{mM}$ GlutaMAX (Invitrogen), $1 \mu \mathrm{M}$ Dexamethasone (10-6 M, Sigma-Aldrich), $10 \mu \mathrm{M}$ insulin (Sigma-Aldrich), 0,5 mM IBMX (3-Isobutyl-1-methylxanthine, Sigma-Aldrich) and $200 \mu \mathrm{M}$ indomethacin (Sigma-Aldrich).

Basic medium consisted of Alfa-MEM (Invitrogen), supplemented with 10 $\%$ FBS (Sigma-Aldrich), $100 \mathrm{U} / \mathrm{ml}$ penicillin and $100 \mu \mathrm{g} / \mathrm{ml}$ streptomycin 
(Invitrogen) and $2 \mathrm{mM}$ GlutaMAX (Invitrogen). Differentiation medium was changed 3 times per week by replacing $2 / 3^{\text {rd }}$ of the medium.

\section{Staining}

Lipid deposition, a marker for adipogenic differentiation, was stained using oil-red-o solution as follows, $3 \mathrm{mg} / \mathrm{ml}$ oil-red-o solution in isopropanol, was just before use diluted to $1,8 \mathrm{mg} / \mathrm{ml}$ with PBS and filtered at $0,2 \mu \mathrm{m}$. The gels were transferred to a mixture of $60 \%$ isopropyl alcohol and $40 \%$ PBS, stained for 5 minutes and washed with PBS until clear.

Mineralization, a marker for osteogenic differentiation, was stained using Alizarin Red. The staining was performed with $2 \mathrm{wt} / \mathrm{v} \%$ alizarin red (SigmaAldrich) solution in $0,9 \% \mathrm{NaCl}$ for 5 minutes and washed with $0,9 \% \mathrm{NaCl}$ until clear.

As an early osteogenic marker alkaline phosphatase (ALP) staining, using fast blue, was performed by dissolving $1,5 \mathrm{mg}$ fast RR salt in 4,8 $\mathrm{ml} \mathrm{PBS}+200 \mu \mathrm{l}$ naphtol AS-MX. The solution was $0,2 \mu \mathrm{m}$ filtered, and gels were stained for 3 hours, and washed until clear with PBS.

\section{Results}

\section{Mechanical properties}

Microgels of dextran-tyramine (Dex) and a combination of dextrantyramine and hyaluronic acid-tyramine (DexHA) were produced in two weight/volume percentages (wt/v \%), resulting in four unique combinations (Figure 1D). Rheological measurements on these materials revealed comparable E-moduli strictly depending on the wt/ $\mathrm{v} \%$ of the polymer conjugates but not on the composition. The E-modulus of $5 \%$ gels was 3-3,5 $\mathrm{kPa}$. Increasing the gel content to $10 \%$ increased the E-modulus to $21 \mathrm{kPa}$ (Figure 1E). Thus, with the same stiffness, the effect of the presence of hyaluronic acid in a dextran hydrogel can be studied. 
A

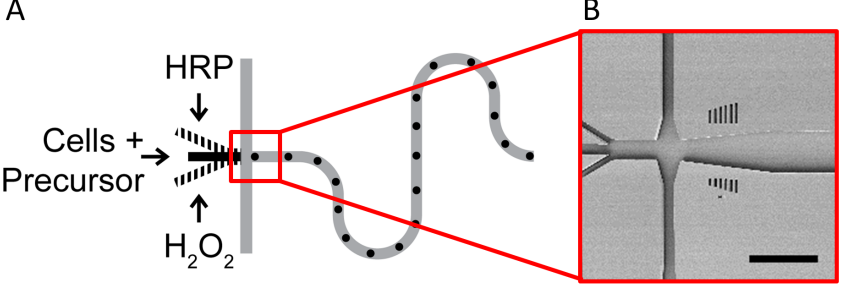

C

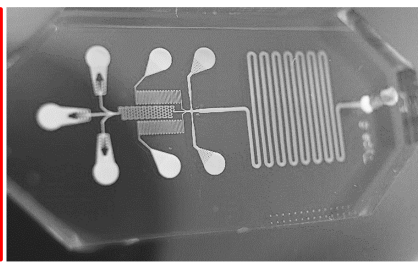

D

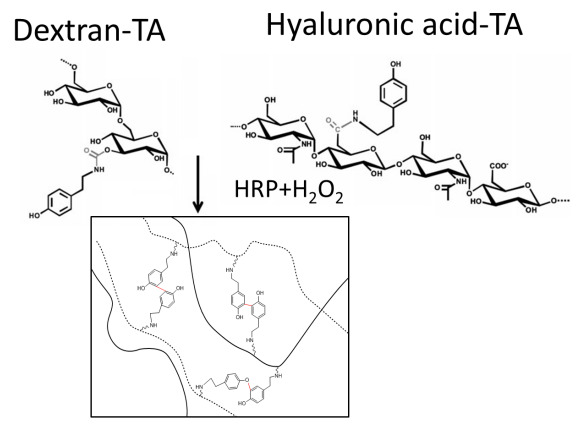

$\mathrm{E}$

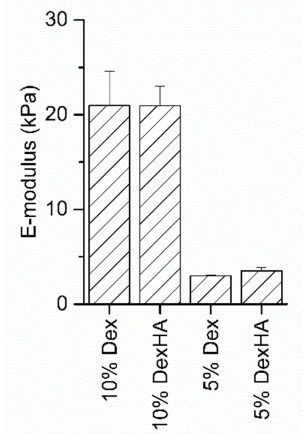

Figure 1 (identical to figure 1 of chapter 4) 1A) Schematic overview of the microfluidic chip with 3 water inlets, containing the polymer precursor with respectively $\mathrm{HRP}$, cells and $\mathrm{H}_{2} \mathrm{O}_{2}$. Vertical inlets are for 2 oil streams, to disperse the water phase into droplets. After droplet formation, the droplets flow through an incubation channel, before collection off chip. 1B) SEM micrograph of the flow focusing region, showing 3 water streams joining just before the flow focusing region. Scale bar represents $500 \mu \mathrm{m} .1 \mathrm{C}$ ) Photograph of the PDMS/glass microfluidic chip, for visualization purposes only the outlet has been punched open. 1D) Tyramine conjugated polymers like dextran-tyramine and hyaluronic acid-tyramine are co-crosslinked into a hydrogel network using horseradish peroxidase and hydrogen peroxide. 1E) Co-crosslinking of Dex-TA and HA-TA results in gels with an E-modulus of 3 to $3,5 \mathrm{kPa}$ for $5 \%$ gels and $21 \mathrm{kPa}$ for $10 \%$ gels, irrespective of the presence of hyaluronic acid-tyramine.

\section{hMSC differentiation}

As shown in Figure 2, the MSC donor used in this study possessed multilineage differentiation capacity in the osteogenic and adipogenic lineage. After 3 weeks of culture in osteogenic medium the MSCs were differentiated in mineralized matrix depositing osteoblasts or when cultured in adipogenic differentiation into fat droplets containing adipocytes (Figure 2). Cells from the same donor were subsequently used for the production of cell laden microgels. These microgels had an average size of $125 \mu \mathrm{m}$ and each contained approximately 10 cells. The shape of the microgels was round with a flat top and bottom due to the height restriction in the microfluidic chip ${ }^{[14]}$. 


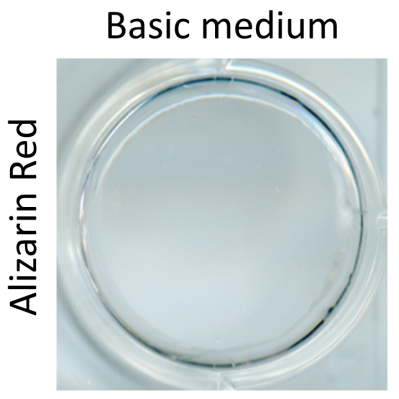

Basic medium

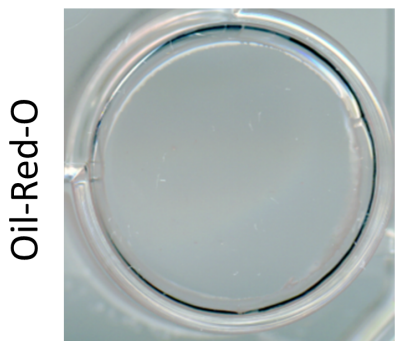

Osteogenic medium
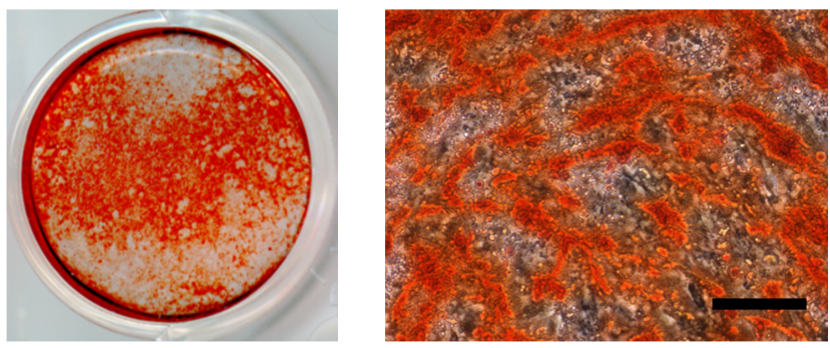

Adipogenic medium
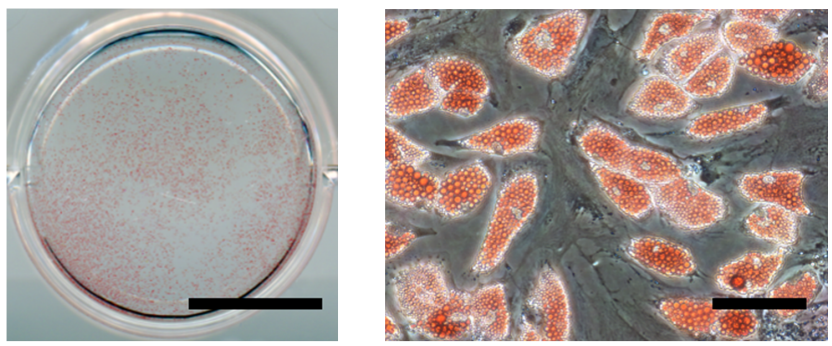

Figure 2: Osteogenic and adipogenic differentiation of hMSCs on tissue culture plastic. Cells are cultured for 3 weeks in osteogenic or adipogenic culture medium. Matrix mineralization is stained red with alizarin red, lipid droplets are stained red with oil-red-o. Scale bar is $500 \mu \mathrm{m}$ for the wholewell images and $100 \mu \mathrm{m}$ for the microphotographs.

Subsequently, approximately 8000 microgels per well corresponding to approximately $8 \mu \mathrm{l}$ of gel were differentiated in adipogenic or osteogenic differentiation medium for a period of 4 weeks. Medium was refreshed three times a week. Microgels were harvested 4 weeks after the start of the differentiation experiment and processed for assessing cell survival using a life dead assay and for cytochemical stainings to visualize the presence of a mineralized extracellular matrix using alizarin red staining, characteristic for mineralizing osteoblasts, or fat droplets that are characteristic for adipocytes. 

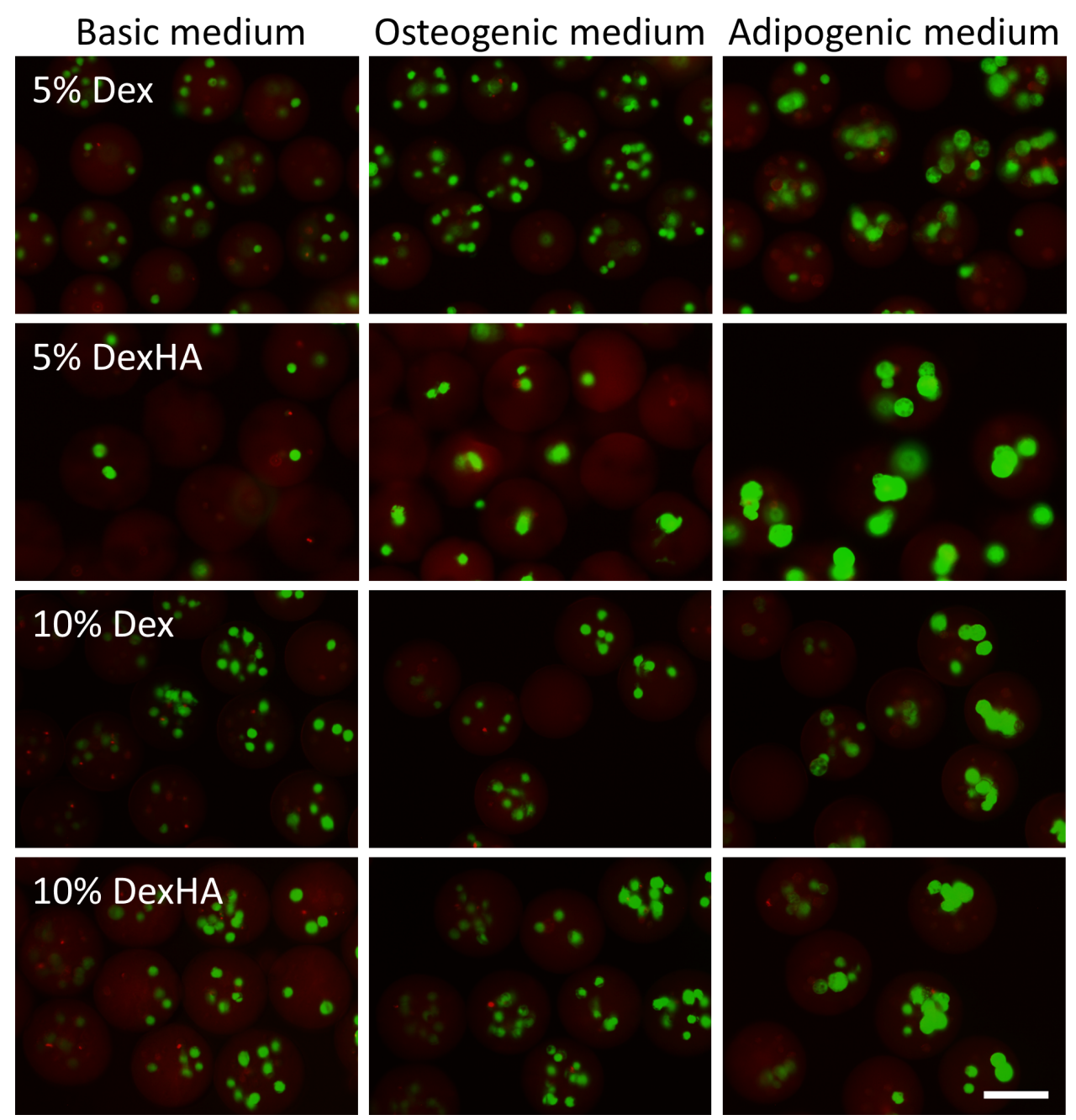

Figure 3: Live/dead staining of encapsulated hMSCs after 4 weeks of culture in basic, osteogenic and adipogenic media. Live cells stain green and dead cells stain red. Scale bar represents $100 \mu \mathrm{m}$.

After 4 weeks of culture cell survival was high. The $10 \mathrm{wt} / \mathrm{v} \%$ gels tended to contain a bit more dead cells, especially in the gels cultured in proliferation medium (Figure 3).

hMSCs in the soft 5\% Dex microgels cultured in osteogenic medium faintly stained with alizarin red (Figure 4), suggesting fairly limited osteogenic differentiation. Remarkably, some cells contained lipid droplets, indicating spontaneous adipogenic differentiation even though cells were cultured in 
medium preferentially favoring osteogenic differentiation (depicted by arrows in Figure 4). The vast majority of the cells in 5\% Dex gels cultured in adipogenic medium stained oil-red-o positive. Both cells with large and small lipid droplet were present. Only few cells did not stain positive for oil-red-o, indicating efficient differentiation in the adipogenic lineage.

Most of the hMSCs cultured for four weeks in 5\% DexHA grew out of the gels, facilitated by the degradation of hyaluronic acid-tyramine. Thus only few cells were left for staining. None of the hMSCs in soft 5\% DexHA gels cultured in osteogenic medium stained positive for alizarin red. In marked contrast, almost all of the remaining cells in the gels cultured in adipogenic medium stained positive for oil-red-o, indicating efficient adipogenic differentiation.

hMSCs encapsulated in the stiff $10 \%$ Dex microgels and cultured in osteogenic medium stained intensely red, indicating good osteogenic differentiation. When cultured in adipogenic medium the majority of the cells stained only slightly oil-red-o positive, compared to cells in the soft $5 \%$ Dex microgels.

The adipogenic differentiation in 10\% DexHA gels was almost identical to the differentiation in $10 \%$ Dex gels. However, the osteogenic differentiation was very different. No cells stained positive for Alizarin red, indicating inhibition of osteogenic differentiation by the hyaluronic acid. 
Osteogenic differentiation

Alizarin Red

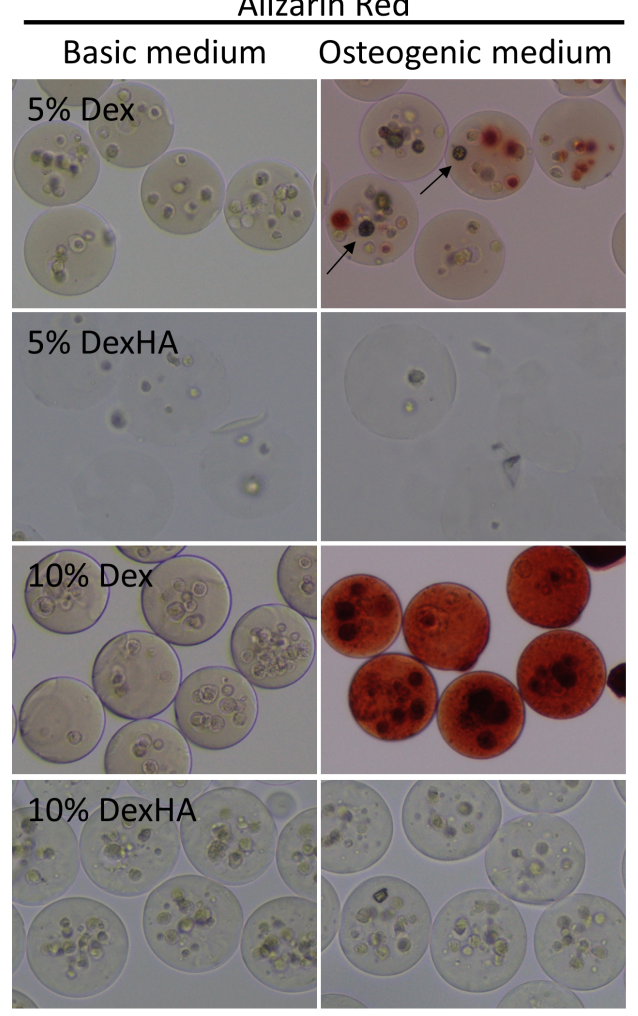

Adipogenic differentiation

Oil red $\mathrm{O}$

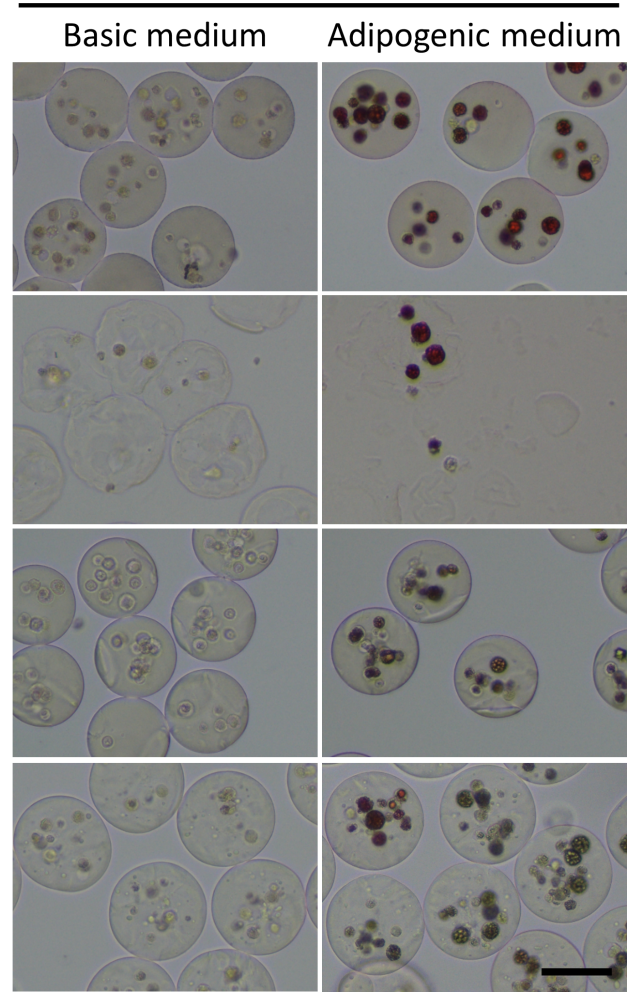

Figure 4: Differentiation analysis of hMSCs encapsulated in 4 types of microgels cultured for four weeks in osteogenic or adipogenic differentiation media. Alizarin red was used for staining of matrix mineralization as a marker of osteogenic differentiation and Oil red $\mathrm{O}$ staining of lipid droplets was used as a marker for adipogenic. Arrows indicate apparent lipid droplets in cells in soft $5 \%$ Dex gels cultured in the adipogenic differentiation medium. Scale bar represents $100 \mu \mathrm{m}$.

\begin{tabular}{|l|l|l|l|l|}
\hline & $5 \%$ Dex & $5 \%$ DexHA & $10 \%$ Dex & $\begin{array}{l}10 \% \\
\text { DexHA }\end{array}$ \\
\hline Stiffness (E-modulus in kPa) & 3 & 3,5 & 21 & 21 \\
\hline Osteogenic differentiation & +- & -- & ++ & -- \\
\hline Adipogenic differentiation & ++ & ++ & +- & +- \\
\hline
\end{tabular}

Since matrix mineralization is a late marker of osteoblast differentiation, we performed a fast blue staining for alkaline phosphatase, an early marker of osteogenesis, in the $10 \mathrm{wt} / \mathrm{v} \%$ Dex and DexHA gels. This to test whether the 
inhibitory effect of hyaluronic acid on matrix mineralization is due to an early inhibitory effect in the osteogenic lineage or is relative late, only affecting matrix mineralization. The majority of the cells in the 10\% Dex gels cultured in osteogenic medium stained positive for ALP. In marked contrast, in the $10 \%$ DexHA however, staining for ALP was neglectable, indicating that the presence of $\mathrm{HA}$ resulted in an early blockade in the osteogenic differentiation pathway.

\section{Early osteogenic differentiation}

\section{Fast Blue}

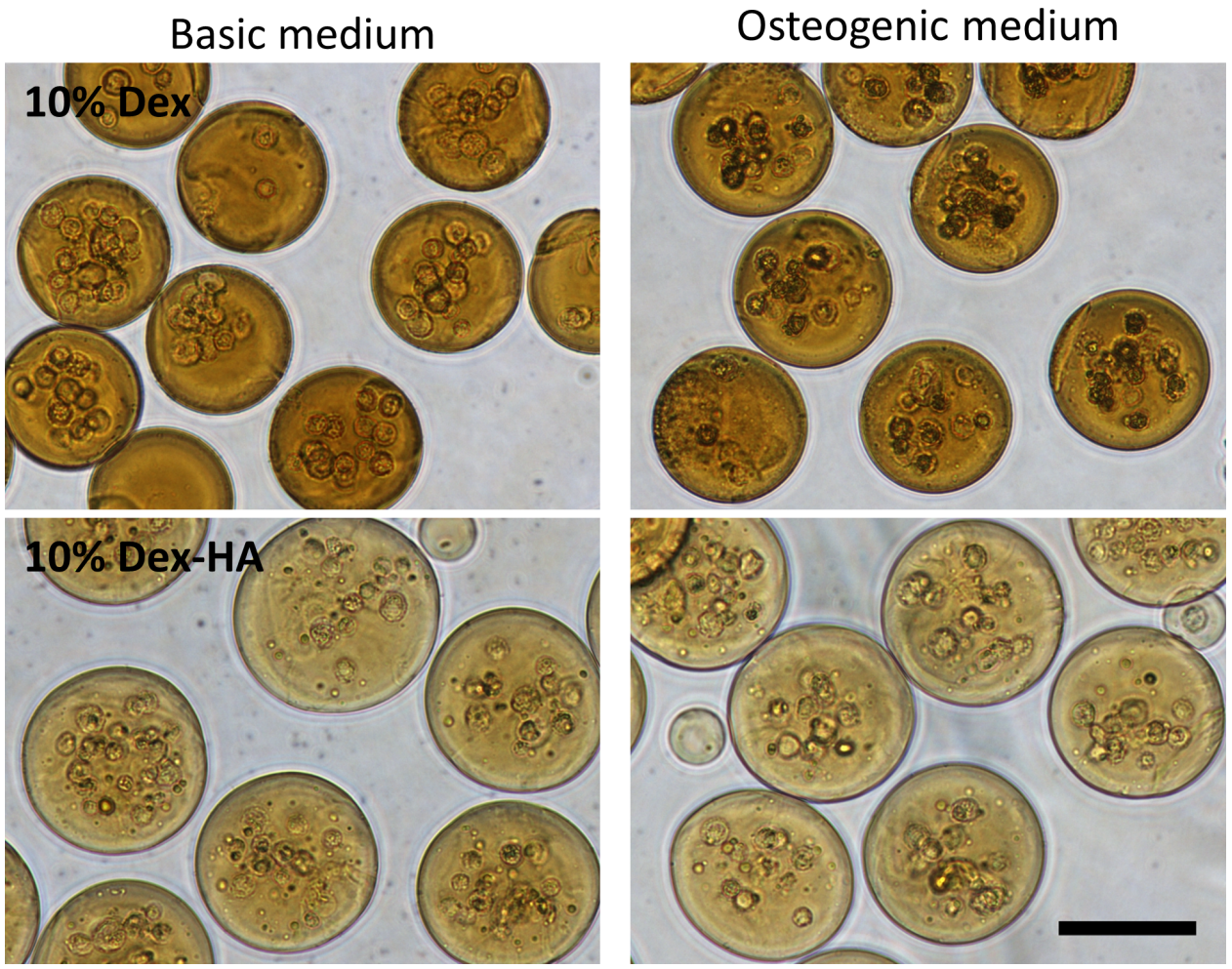

Figure 5: Fast blue staining of MSCs encapsulated in $10 \%$ Dex or $10 \%$ DexHA microgels cultured for 4 weeks in osteoblastic differentiation medium for visualization of the early bone marker alkaline phosphatase. Scale bar represents $100 \mu \mathrm{m}$. 


\section{Discussion}

Previously, numerous studies have shown that matrix stiffness controls MSC differentiation, however, most of these studies are done using 2D culture models. The effect of matrix stiffness in a 3D microenvironment is considerably less studied. In general, the trend found in 2D studies does match with a fairly limited number of studies that were performed in 3D. In this study we successfully encapsulated MSCs in enzymatically crosslinked hydrogels. We subsequently tested whether the microencapsulated MSCs could act as building blocks for bottom-up tissue engineering strategies by studying the survival and differentiation capacity of these cells in these microenvironments. To create the building blocks we used gels composed of pure Dex-TA or of a 1:1 mixture of DexTA and HA-TA at a wt/v \% of 5 and $10 \%$. Rheological measurements suggested that the stiffness of the microgels was strictly dependent on $\mathrm{wt} / \mathrm{v} \%$ of the polymer conjugates irrespective of its composition. This allowed us to study the effect on MSC differentiation of the composition of the microenvironment independent of stiffness. The stiffness of the microgels is extrapolated from rheological measurements in macro gels, as previous measurements on microgels composed of thiol-modified HA using AFM suggested that the measurements in macro and in microgels are largely in line ${ }^{[15]}$. However, it is advised to study the rheological properties at the level of the individual microgels using AFM.

By changing the wt $/ v \%$ we not only change stiffness but also the mesh size of the hydrogel. These parameters are closely interrelated. However, a decrease in mesh size may negatively impact the diffusion of nutrients and waste products. Such difference may potentially explain differences in differentiation characteristics between the two gel types. However, one would expect that in the $10 \mathrm{wt} / \mathrm{v} \%$ gels this would have impacted cell behavior for example by showing more cell death in long term culture, reduced differentiation capacity, or diminished metabolic activity. Detailed analysis of the various cell-laden gel types did not demonstrate obvious differences in each of these cell functions even in cultures up to 4 weeks. Although diffusion might be slightly hampered in the 10 $\mathrm{wt} / \mathrm{v} \%$ gels, this did not affect MSC differentiation, suggesting that the observed differences are most likely explained by differences in stiffness and composition of the microenvironments. It would be advisable to study the impact on diffusion of macromolecules by changing $\mathrm{wt} / \mathrm{v} \%$ of the microgels. 
When MSCs are embedded in pure Dex microgels and cultured in adipogenic or osteogenic medium, results consistent with literature are found. In soft gels (3-3,5 kPa) cells predominantly differentiate into adipocytes while in the stiff hydrogels $(21 \mathrm{kPa})$ the cells efficiently differentiate into mineralizing osteoblasts. This raises an intriguing question: How do cells sense the stiffness of the microenvironment in a pure Dex gel? This is relevant since human cells lack receptors that can specifically interact with dextran. In previous work it has been demonstrated that cells sense the stiffness of the extracellular matrix via integrin receptors which couple the extracellular with the intracellular world. Via a process called mechanotransduction pulling forces of the cells on the extracellular matrix are translated into intracellular biochemical signals that ultimately result in activation of lineage specific genetic networks. Our results suggest that, besides signaling via integrins, additional mechanisms exist by which cells can sense the stiffness of the extracellular matrix. For example, it seems feasible that filopodia are entangled by the hydrogel during the in situ gelation process resulting in a physical anchoring of the cell in the hydrogel. These entanglements could serve as equivalents of integrin mediated focal adhesions. This hypothesis is currently under investigation. Alternative explanations are also possible and may all point to a new mechanism for mechanotransduction.

When adding hyaluronic acid-tyramine, the matrix becomes partially degradable. In case of the $5 \%$ gels, after four weeks most cells have escaped from the gels. The remaining cells show good adipogenic differentiation. Strikingly, addition of hyaluronic acid-tyramine inhibits the differentiation of MSCs into mineralized matrix depositing osteoblasts, both in soft and in stiff gels. To our knowledge, this inhibition of hyaluronic acid on osteogenic differentiation of MSCs has not been reported in literature. Since matrix mineralization is a late marker for osteogenic differentiation, we cannot distinguish whether this effect is due to a complete block of osteoblast differentiation, a temporary arrest in differentiation or is merely a consequence of a delay in differentiation. Based on our observation that also the expression of the early bone marker alkaline phosphatase is clearly inhibited, we propose that the presence of HA can arrest osteoblast differentiation in a very early phase of stem cell differentiation. The exact mechanism by which this inhibitory effect is exerted deserves further study. 
Particularly since a few studies have successfully used HA based gels as support material for osteogenic differentiation of MSCs ${ }^{[9,15,21-23]}$.

Compared to other work, we use relatively low molecular weight HA (16 $\mathrm{kDa}{ }^{[15,23]}$. These short chains of HA might have different effects compared to more common higher molecular weight HA as described in literature. For example low and high molecular weight $\mathrm{HA}$ have different effects on induction of vascularization ${ }^{[24]}$. A remarkable difference with other studies in which HA based gels have been used, is the relative low degree of substitution of carboxylic acid groups with, in our case, tyramine residues, on the hyaluronic acid. The introduction of these functional groups is needed to enable the chemical crosslinking of individual HA molecules into stable macromolecular networks. In our study only 2 out of every 100 units is functionalized with a tyramine group. This is in marked contrast to studies by others in which degrees of substitution of $25 \%$ or more have been used ${ }^{[15,23]}$. In addition, in these studies the carboxylic acid residues have been substituted with thiol or methacrylate groups to render a crosslinkable hydrogel. It seems likely that the high degree of substitution drastically alters the chemical properties of HA and this might change the way how cells recognize and respond to this material. Cells interact with HA via the CD44 transmembrane receptor. It would be of interest to examine whether the degree of substitution as well as the molecular weight of HA influences binding of HA to CD44 and subsequent signal transduction pathways.

Another interesting finding of this study is our observation that differentiation signals provided by matrix stiffness and culture medium can be completely overruled by changing the composition of the microenvironment without changing stiffness. The blocking signal on osteoblastic differentiation exerted by HA clearly antagonizes the signals induced by matrix stiffness, in particular for osteoblastic differentiation. HA did however not block adipocyte differentiation. This suggests that the inhibitory effect of HA on MSC differentiation as observed in this study is lineage specific.

In conclusion, this study shows the ability for using microfluidics to engineer specialized cell-laden microgels in which vary in hydrogel composition and stiffness. The ability to tune the composition and the stiffness of the microgels on this platform enables the creation of specialized microenvironments for the bottom-up tissue engineering of functional tissue constructs. 


\section{References}

[1] J. Leijten, J. Rouwkema, Y. S. Zhang, A. Nasajpour, M. R. Dokmeci, and A. Khademhosseini, "Advancing Tissue Engineering: A Tale of Nano-, Micro-, and Macroscale Integration", Small, vol. 12, no. 16, pp. 2130-2145, 2016.

[2] D. L. Elbert, "Bottom-up tissue engineering", Current Opinion in Biotechnology, vol. 22, no. 5, pp. 674-680, 2011.

[3] Y. T. Matsunaga, Y. Morimoto, and S. Takeuchi, "Molding Cell Beads for Rapid Construction of Macroscopic 3D Tissue Architecture", Advanced Materials, vol. 23, no. 12, pp. H90-H94, 2011.

[4] Y. Du, E. Lo, S. Ali, and A. Khademhosseini, "Directed assembly of cell-laden microgels for fabrication of 3D tissue constructs", Proceedings of the National Academy of Sciences, vol. 105, no. 28, pp. 9522-9527, 2008.

[5] M. F. Pittenger, A. M. Mackay, S. Beck, R. K. Jaiswal, R. Douglas, J. D. Mosca, M. a. Moorman, D. W. Simonetti, S. Craig, and D. Marshak, "Multilineage Potential of Adult Human Mesenchymal Stem Cells", Science, vol. 284, no. April, pp. 143-147, 1999.

[6] J. S. Park, J. S. Chu, A. D. Tsou, R. Diop, Z. Tang, A. Wang, and S. Li, "The effect of matrix stiffness on the differentiation of mesenchymal stem cells in response to TGF- $\beta$ ", Biomaterials, vol. 32, no. 16, pp. 3921-3930, 2011.

[7] C. Frantz, K. M. Stewart, and V. M. Weaver, "The extracellular matrix at a glance.", Journal of cell science, vol. 123, pp. 4195-4200, 2010.

[8] S. Gobaa, S. Hoehnel, M. Roccio, A. Negro, S. Kobel, and M. P. Lutolf, "Artificial niche microarrays for probing single stem cell fate in high throughput", Nature Methods, vol. 8, no. 11, pp. 949-955, 2011.

[9] W. Zhao, X. Li, X. Liu, N. Zhang, and X. Wen, "Effects of substrate stiffness on adipogenic and osteogenic differentiation of human mesenchymal stem cells", Materials Science and Engineering C, vol. 40, pp. 316-323, 2014.

[10] A. J. Engler, S. Sen, H. L. Sweeney, and D. E. Discher, "Matrix Elasticity Directs Stem Cell Lineage Specification", Cell, vol. 126, no. 4, pp. 677-689, 2006.

[11] A. G. Cristancho and M. A. Lazar, "Forming functional fat: a growing understanding of adipocyte differentiation.", Nature reviews. Molecular cell biology, vol. 12, no. 11, pp. 722-34, 2011.

[12] Y. R. V Shih, K. F. Tseng, H. Y. Lai, C. H. Lin, and O. K. Lee, "Matrix stiffness regulation of integrin-mediated mechanotransduction during osteogenic differentiation of human mesenchymal stem cells", Journal of Bone and Mineral Research, vol. 26, no. 4, pp. 730-738, 2011.

[13] L. S. Moreira Teixeira, J. Feijen, C. a. van Blitterswijk, P. J. Dijkstra, and M. Karperien, "Enzyme-catalyzed crosslinkable hydrogels: Emerging strategies for tissue engineering", Biomaterials, vol. 33, no. 5, pp. 1281- 
1290, 2012.

[14] S. Henke, J. Leijten, E. Kemna, M. Neubauer, A. Fery, A. van den Berg, A. van Apeldoorn, and M. Karperien, "Enzymatic Crosslinking of Polymer Conjugates is Superior over Ionic or UV Crosslinking for the On-Chip Production of Cell-Laden Microgels", Macromolecular Bioscience, vol. 16, no. 10, pp. 1524-1532, 2016.

[15] Y. Ma, M. P. Neubauer, J. Thiele, A. Fery, and W. T. S. Huck, "Artificial microniches for probing mesenchymal stem cell fate in 3D", Biomaterials Science, 2014.

[16] R. Xue, J. Y.-S. Li, Y. Yeh, L. Yang, and S. Chien, "Effects of matrix elasticity and cell density on human mesenchymal stem cells differentiation.", Journal of orthopaedic research : official publication of the Orthopaedic Research Society, vol. 31, no. 9, pp. 1360-5, 2013.

[17] C.-C. Lin and K. S. Anseth, "Cell-cell communication mimicry with poly(ethylene glycol) hydrogels for enhancing -cell function", Proceedings of the National Academy of Sciences, vol. 108, no. 16, pp. 6380-6385, 2011.

[18] J. W. H. Wennink, K. Niederer, A. I. Bochyńska, L. S. Moreira Teixeira, M. Karperien, J. Feijen, and P. J. Dijkstra, "Injectable Hydrogels by Enzymatic Co-Crosslinking of Dextran and Hyaluronic Acid Tyramine Conjugates", Macromolecular Symposia, vol. 309-310, no. 1, pp. 213-221, 2011.

[19] S. Henke, J. Leijten, T. Kamperman, M. Groot Nibbelink, R. Wang, J. Plass, A. Tons, M. Hanegraaf, F. Carlotti, E. de Koning, P. J. Dijkstra, A. Van Apeldoorn, and M. Karperien, "Proliferation restrictive microenvironments for beta cell encapsulation", In Preparation.

[20] S. K. Both, A. J. C. van der Muijsenberg, C. a van Blitterswijk, J. de Boer, and J. D. de Bruijn, "A rapid and efficient method for expansion of human mesenchymal stem cells", Tissue Eng, vol. 13, no. 1, pp. 3-9, 2007.

[21] A. K. Jha, X. Xu, R. L. Duncan, and X. Jia, "Controlling the adhesion and differentiation of mesenchymal stem cells using hyaluronic acid-based, doubly crosslinked networks", Biomaterials, vol. 32, no. 10, pp. 24662478, 2011.

[22] L. Zou, X. Zou, L. Chen, H. Li, T. Mygind, M. Kassem, and C. Bünger, "Effect of hyaluronan on osteogenic differentiation of porcine bone marrow stromal cells in vitro.", Journal of orthopaedic research: official publication of the Orthopaedic Research Society, vol. 26, no. 5, pp. 71320, 2008.

[23] S. Khetan, M. Guvendiren, W. R. Legant, D. M. Cohen, C. S. Chen, and J. a Burdick, "Degradation-mediated cellular traction directs stem cell fate in covalently crosslinked three-dimensional hydrogels.", Nature materials, vol. 12, no. 5, pp. 458-65, 2013. 
[24] P. Rooney, S. Kumar, J. Ponting, and M. Wang, "The role of hyaluronan in tumour neovascularization (review)", International Journal of Cancer, vol. 60, no. 5, pp. 632-636, 1995. 


\section{Chapter 4}

\section{Proliferation restrictive micro-environments for beta cell encapsulation}

Sieger Henke, Jeroen Leijten, Tom Kamperman, Milou Groot Nibbelink, Rong Wang, Jacqueline Plass, Annemieke Tons, Maaike Hanegraaf, Francoise Carlotti, Eelco de Koning, Pieter J Dijkstra, Aart van Apeldoorn, Marcel Karperien 


\section{Abstract}

Transplantation of iPSC derived beta cells holds great promise for treatment of type 1 diabetes. However, differentiation of cells in the transplant is never $100 \%$, risking teratocarcinoma formation by undifferentiated cells. Transplantation of cells in a proliferation restrictive microenvironment may help to solve this problem. In this study we explored the potential of cell-laden enzymatically crosslinked microgels composed of natural polymer-tyramine conjugates to generate such proliferation restrictive environments. The MIN6 beta cell line was used as a model for aggressive proliferating cells with beta cell characteristics. Gels were produced of combinations dextran-tyramine and hyaluronic acid-tyramine conjugates. The stiffness of the microgel was tuned between $3-3,5 \mathrm{kPa}$, which is close to the physiological stiffness of the native islet, and $21 \mathrm{kPa}$.

Cell survival after encapsulation was excellent in all microgel types. We found that stiff microgels effectively inhibited MIN6 cell proliferation, with the strongest effect of the bioinert dextran-tyramine gels. Inhibition of proliferation did not impair glucose responsiveness as determined by insulin secretion. In vivo implantation under the kidney capsule showed vascularization of the grafts already after 2 weeks. Non-encapsulated MIN6 cells proliferated unrestricted and invaded half of the kidney within 2 weeks. Soft gels with hyaluronic acid-tyramine inhibited cell proliferation, but outgrowth from the gels was present, suggesting decreased stability of soft microgels. In contrary, both the soft gels without hyaluronic acid-tyramine, and stiff gels with hyaluronic acid-tyramine effectively contained the cells in the microgel, while the cells remained insulin positive.

In conclusion, unrestricted proliferation of transplanted beta-like cells can be effectively counteracted by encapsulating the cells in a stiff microgel of dextran-tyramine and a combination of dextran-tyramine/hyaluronic acidtyramine (1:1) in vitro and in vivo without impairing insulin positivity. This strategy enables the design of custom, proliferation restricting environments, tuned to the needs of the encapsulated cells and holds great promise for transplantation of iPSC derived beta cells to prevent teratocarcinoma formation. 


\section{Introduction}

Type 1 diabetes is a chronic disease with no definitive cure available for the majority of patients ${ }^{[1]}$. Management of the disease is usually done by frequent blood glucose measurements and insulin injections. Although this works for most patients relatively well, it is very burdensome and will lead to complication in the long run. A promising cure is islet transplantation, but the availability is very limited due to organ shortage. Additionally, it requires the use of systemic immunosuppressants, which is problematic on the long term ${ }^{[2]}$. Thus alternative beta cell sources for transplantation purposes are necessary. In the past years, a great advance in induced pluripotent stem cell (iPSC) technology made it possible to differentiate iPSCs in vitro into fully functional beta cells that can normalize blood glucose levels when transplanted in a diabetic mouse model [3].

However, IPSC therapy has the inherent risk of transplanting undifferentiated cells that may induce the formation of teratocarcinomas ${ }^{[4]}$. Hence there is a need for devices that can be used for beta cell transplantation that can prevent uncontrolled proliferation of undifferentiated iPSC that remained present in the transplant. Multiple solutions for this problem are possible and are currently explored. For example macro encapsulation of the iPSC derived beta cells in a "bag" ${ }^{[5]}$ or in a "solid" scaffold ${ }^{[6]}$. An alternative solution could be the encapsulation of the iPSC derived beta cells in a proliferation restrictive microenvironment. Beta cell encapsulation in alginate is already frequently used particularly for its immunoprotective properties ${ }^{[7]}$. This approach could also be used for creating a proliferation restrictive environment. However, alginate microgels are relatively large and dense, thus causing some limitation in diffusion of oxygen, nutrients and waste products ${ }^{[8]}$, and are unstable over time ${ }^{[9]}$ which make them less suited for preventing uncontrolled proliferation in the long run, since after collapse of the microgels the restriction is gone.

We have previously reported on an enzymatically crosslinkable hydrogel system, of which both mechanical and chemical properties can be independently tuned. The hydrogel consists of crosslinkable polysaccharide tyramine conjugates like dextran-tyramine (Dex-TA) and hyaluronic acid-tyramine (HA-TA) in varying concentrations ${ }^{[10,11]}$. Dextran is a natural polymer with proven clinical safety, which is not actively degradable in the human body, because the enzyme 
dextranase is not present. In the human body it can only be degraded by hydrolysis, which is a very slow process ${ }^{[12]}$. It can successfully be used for encapsulation of cells in micro hydrogels (microgels) ${ }^{[13]}$. A drawback of Dex-TA based hydrogels is that the material doesn't allow for cell interaction since human cells lack receptors interacting with dextran. This can be solved by replacing (part) of the Dex-TA with HA-TA, which allows for cell interaction via the CD44 receptor. Based on studies that show CD44 mediated processes in diabetes and pancreatic tumors ${ }^{[14]}$, it is likely that CD44 is expressed in native beta cells. Its role in beta cell physiology is still largely unknown.

Next to the material composition, the stiffness of the matrix surrounding an encapsulated cell is directly relevant for normal cell behavior. Native pancreas stiffness is not exactly known and reports range from $1,2 \mathrm{kPa}{ }^{[15]}, 1,4 \mathrm{kPa}{ }^{[16]}$, to $8,25 \mathrm{kPa}{ }^{[17]}$. In this study we include two stiffnesses, one in the order of magnitude of native pancreatic tissue (3-3,5 $\mathrm{kPa}$ ), and one several fold higher (20 $\mathrm{kPa})$. It has been shown previously that a more stiff microenvironment is expected to decrease proliferation capacity of embedded cells ${ }^{[18]}$. We use the MIN6 beta cell line as model. These cells are relatively aggressive tumor beta cells, with an in vitro doubling time of 36-48 hours. Furthermore they are glucose responsive and respond with insulin secretion. We hypothesize that if successful encapsulation and proliferation limitation works with MIN6 while retaining glucose responsiveness and insulin secretion, microencapsulation in a proliferation restrictive microenvironment may also prevent uncontrolled proliferation of undifferentiated iPSC cells in a beta cell transplant. We tested our hypothesis in vitro and in vivo by transplantation of microgels with encapsulated MIN6 cells under the kidney capsule, studying cell proliferation and glucose dependent insulin secretion.

\section{Materials and Methods}

\section{Hydrogel material}

Dextran-tyramine (MW $18460 \mathrm{~g} / \mathrm{mol}$ (15-30 kg/mol Sigma-Aldrich)) and hyaluronic acid-tyramine (MW $16000 \mathrm{~g} / \mathrm{mol}$ (15-30 kg/mol Contipro)) conjugates were prepared as described previously ${ }^{[10]}$. Dex-TA was used with a degree of substitution (DS, number of tyramine residues per 100 disaccharide blocks) of 15, 
HA-TA with a DS of 2. $10 \%$ Gels contained $28,6 \mathrm{U} / \mathrm{ml} \mathrm{HRP}$ and 0,016 $\mathrm{mmol} / \mathrm{ml}$ $\mathrm{H}_{2} \mathrm{O}_{2}, 5 \%$ gels contained $14,3 \mathrm{U} / \mathrm{ml} \mathrm{HRP}$ and $0,008 \mathrm{mmol} / \mathrm{ml} \mathrm{H}_{2} \mathrm{O}_{2}$.

\section{Rheology}

$250 \mu \mathrm{l}$ gels were prepared on ice in a glass round bottom tube and placed on room temperature to allow for gelation. Gelated gels were transferred to a flat plate rheometer (Anton Paar, MCR 301, $25 \mathrm{~mm}$ flat plates), and measured with a gap size of $250 \mu \mathrm{m}$, frequency of $0,5 \mathrm{~Hz}$ and a strain of $0,1 \%$. The $E$ modulus was calculated from the storage modulus $\mathrm{G}$, assuming a linear elastic material and Poisson's ratio $v=0,5$ as follows:

$$
E=2 G^{\prime}(1+v)
$$

\section{Cell culture}

MIN6B1 cells were cultured in DMEM (Invitrogen), supplemented with 10 $\%$ heat-inactivated FBS (Sigma-Aldrich), 71 MM 2-mercaptoethanol (Invitrogen) and $100 \mathrm{U} / \mathrm{ml}$ penicillin and $100 \mu \mathrm{g} / \mathrm{ml}$ streptomycin (Invitrogen) and used from passage 30 to 40 . Medium was changed 2 to 3 times a week.

\section{Microfluidic chip preparation}

Microgels were formed using a microfluidic device (Figure 1C). The device was prepared using soft lithography techniques as described previously ${ }^{[13]}$. In short: the chip was designed using CAD software, and the design was transferred to a photomask. Subsequently, a $70 \mu \mathrm{m}$ layer of SU-8 polymer was applied on a silicon wafer and photolithography was performed. PDMS (Sylgard 184, Dow Corning) was mixed 10:1 base:curing agent, applied on the wafer and cured. The PDMS was removed from the wafer, cut to size, after which holes were punched for inlets and outlet. The PDMS microchip was bound to a glass slide after treatment with oxygen plasma.

\section{Microgel formation}

Fluids were infused using syringe pumps. The dispersed aqueous phase consisted out of 3 components: the polymer solution containing the cells and $8 \%$ Optiprep (Sigma-Aldrich), polymer solution containing HRP and polymer solution containing a small amount of hydrogen peroxide. Directly after joining of the three components droplets of approximately $100 \mu \mathrm{m}$ were formed in a flow 
focusing geometry using hexadecane (Sigma-Aldrich) with 1 \% Span 80 (SigmaAldrich) as the continuous phase. A $140 \mathrm{~mm}$ delay channel was used for gelation of the droplets in stable microgels. The microgels were collected via tubing in culture medium. The infusion rate was $50 \mu \mathrm{l} / \mathrm{min}$ of the continuous oil phase and $7 \mu \mathrm{l} / \mathrm{min}$ of the polymer solution (water phase). This resulted in approximately 6*10 $10^{5}$ droplets per hour. 4 types of gels were produced: $10 \mathrm{wt} / \mathrm{v} \%$ Dex-TA (10\% Dex), $10 \mathrm{wt} / \mathrm{v} \%$ Dex-TA/HA-TA (50:50 \%, 10\% DexHA), 5 wt/v \% Dex-TA (5\% Dex) and $5 \mathrm{wt} / \mathrm{v} \%$ Dex-TA/HA-TA (50:50\%, 5\% DexHA)

\section{Cell viability analysis}

The cell viability was assessed with the LIVE/DEAD kit (Invitrogen) according to manufacturer's protocol. Images were taken on an EVOS digital microscope (Westburg/Invitrogen).

\section{Brightfield microscopy and analysis}

Brightfield and phase contrast images were taken on a Nikon Eclipse TE300 microscope equipped with Nikon Digital Sight DS-fi1 camera. Gel size analysis was performed using a custom made Matlab (MathWorks software) script. Cluster size analysis was performed using ImageJ.

\section{Metabolic activity analysis}

Metabolic activity of the encapsulated cells was assessed on day 1, 7 and 14 , using the Prestoblue assay (Invitrogen), according to manufacturer's protocol.

\section{Proliferation analysis}

EdU was added to the culture medium (final concentration $10 \mu \mathrm{M}$ ) and after 24 hours the samples were fixed with $10 \%$ neutrally buffered formalin. Subsequent staining was performed according to manufacturer's protocol (ClickiT EdU, Invitrogen), nuclei were counterstained using DRAQ5 (Invitrogen). Confocal slices were made using a Nikon A1 confocal system. From each condition $\mathrm{n}=7$ gels were analyzed, using 5 consecutive confocal slices per gel. 


\section{Glucose induced insulin secretion test}

The functionality of MIN6 cells in microgels was tested by subsequent incubation in modified Krebs buffer (115 mM NaCl, $5 \mathrm{mM} \mathrm{KCl}, 24 \mathrm{mM} \mathrm{NaHCO}_{3}$, 2,2 $\mathrm{mM} \mathrm{CaCl}_{2}$, 20mM HEPES, 0,3\% Bovine serum albumin, 0,1 mM Theopyllin, $\mathrm{pH}$ $7,4)$ containing Low $(1,67 \mathrm{mM})$ and High $(16,7 \mathrm{mM})$ glucose concentration. Secreted insulin in the buffer was detected by ELISA (Mercodia).

\section{CD44 staining}

CD44 immunofluorescent staining was performed using monoclonal rat anti-mouse CD44 IgG2b (Abcam) primary antibody and mouse monoclonal antirat IgG2b FITC-conjugated (Abcam) secondary antibody. As isotype control a Rat IgG2b isotype control (Abcam) was used. Imaging was performed using a Nikon A1 confocal microscope.

\section{Microaggregate formation}

Microaggregates of 100 cells were formed using an in-house developed system for controlled cell aggregation ${ }^{[19,20]}$. In short, cells were seeded on agarose microchips, and settled into the wells by centrifugation. Cells were allowed to aggregate for 2 days before use.

\section{In vivo implantation}

5\% Dex, 10\% DexHA and 5\% DexHA microgels were implanted under the kidney capsule of 8-12 week old NOD.SCID mice. After two weeks the mice were sacrificed, the kidneys explanted and fixated in $10 \%$ formalin at 4 degrees for 24 hours. Subsequently the kidneys were routinely processed for histology (tissue processor, Themoscientific) and embedded in paraffin.

\section{Histology}

$5 \mu \mathrm{m}$ thick sections were made, applied on superfrost plus slides, and stained with hematoxylin and eosin, and insulin (HRP/DAB) combined with Masson's trichrome. Slides were imaged using a slide scanner (Nanozoomer, Hammamatsu). 


\section{Statistics}

All experiments were conducted with $n=3$ samples, unless stated otherwise. Data were analysed for statistical significance using ANOVA with Bonferroni post hoc test, or with the Kruskal-Wallis test for non-normal distributed data, with a p-value of 0,05 .

A

B

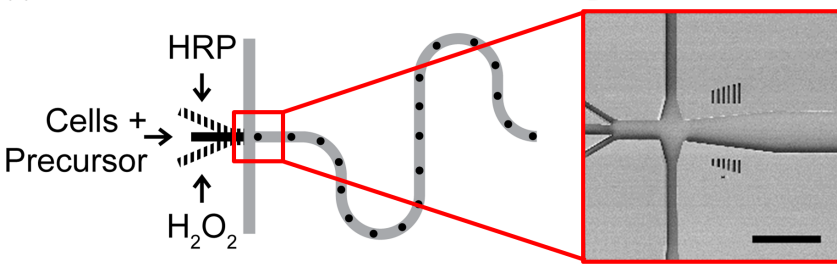

$\mathrm{D}$

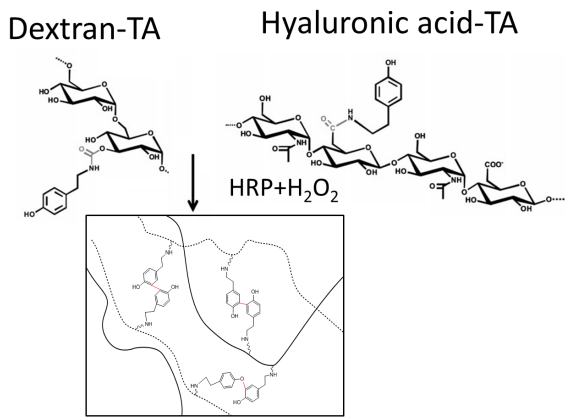

C

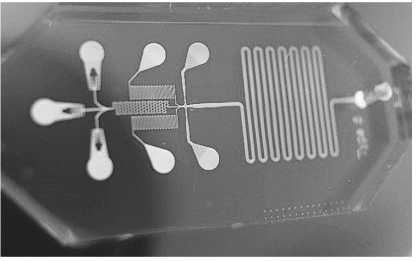

$\mathrm{E}$

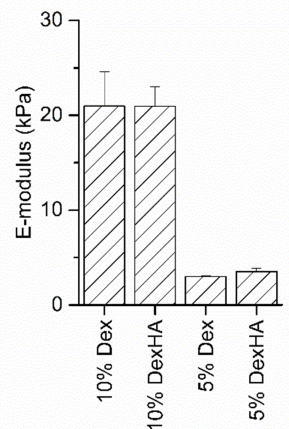

Dex-TA/HA-TA

Figure 1: $1 \mathrm{~A})$ Schematic overview of the microfluidic chip with 3 water streams, containing the polymer precursor with respectively HRP, cells and $\mathrm{H}_{2} \mathrm{O}_{2}$. Vertical inlets are for 2 oil streams, to disperse the water phase into droplets. After droplet formation, the droplets flow through an incubation channel, before collection off chip. 1B) SEM micrograph of the flow focusing region, showing 3 water streams joining just before the flow focusing region. Scale bar represents $500 \mu \mathrm{m}$. 1C) Photograph of the PDMS/glass microfluidic chip, for visualization purposes only the outlet has been punched open. 1D) Tyramine conjugated polymers like dextran-tyramine and hyaluronic acidtyramine are co-crosslinked into a hydrogel network using horseradish peroxidase and hydrogen peroxide. 1E) Co-crosslinking of Dex-TA and HA-TA results in gels with an E-modulus of $21 \mathrm{kPa}$ for $10 \%$ gels and 3 to $3,5 \mathrm{kPa}$ for $5 \%$ gels, irrespective of the polymer composition.

\section{Results}

Using the microfluidic platform depicted in Figure 1, four different material compositions were used for the encapsulation of MIN6 cells in microgels. The four materials consisted of 10\% Dex, 10\% DexHA, 5\% Dex and 5\% DexHA. 
Rheological measurements showed that both $10 \%$ gels resulted in an E-modulus of approximately $21 \mathrm{kPa}$ and both $5 \%$ gels resulted in an E modulus of 3-3,5 kPa, as show in Figure $1 \mathrm{E}$. Thus the mechanical properties of the 4 different gels were solely dependent on the $\mathrm{wt} / \mathrm{v} \%$ of the polymer conjugates rather than the composition of the polymer mixtures. This enabled us to test the effect of hyaluronic acid on behavior of the MIN6 cells without affecting the stiffness of the microenvironment, which is known to influence cell behavior.

With each of the material compositions cell laden microgels were efficiently formed. The gels displayed a round shape (Figure 2A top panel). The shape was uniform with exception of the $10 \%$ DexHA microgels, which showed slightly more variability likely due to the higher viscosity of the DexHA solution. The HA-TA containing microgels tended to be a bit larger than the pure Dex-TA hydrogels. This could either be explained by shrinkage of Dex-TA after crosslinking (data not shown), or by relatively larger swelling of the DexHA gels ${ }^{[10]}$, or the combination of both.

Irrespective of the gel composition, cell survival 4 hours after microgel production was high, exceeding more than $90 \%$ (Figure 2B). This is even an underestimation, as all dead cells in microgels are counted, in contrast to the living cells because of 2D imaging in combination with high cell density, which results in cells in different depths presenting as one.

Remarkably the metabolic activity measured after 1 day (Figure 2C) was dependent on the $\mathrm{wt} / \mathrm{v} \%$ of the polymer concentration, as well as the presence of HA-TA. Cells were metabolically more active in the $5 \%$ gels with low stiffness. The presence of HA-TA even further increased metabolic activity.

When looking at the time points at day 7 and day 14 compared to day 1 (figure 2D), the metabolic activity of cells in 10\% Dex-TA decreased to below 15 $\%$ of the initial value within 14 days. However, when adding HA-TA, metabolic activity increased to $300 \%$ in 14 days. Metabolic activity of cells in softer gels of $5 \%$ Dex-TA first slightly increased towards day 7 , and then dropped to $60 \%$ after 14 days. In $5 \%$ DexHA the already high metabolic activity of day 1 increased to over $300 \%$ at day 7 and passed $400 \%$ at day 14 . 


\section{A} 10\% Dex 10\% DexHA 5\% Dex 5\% DexHA
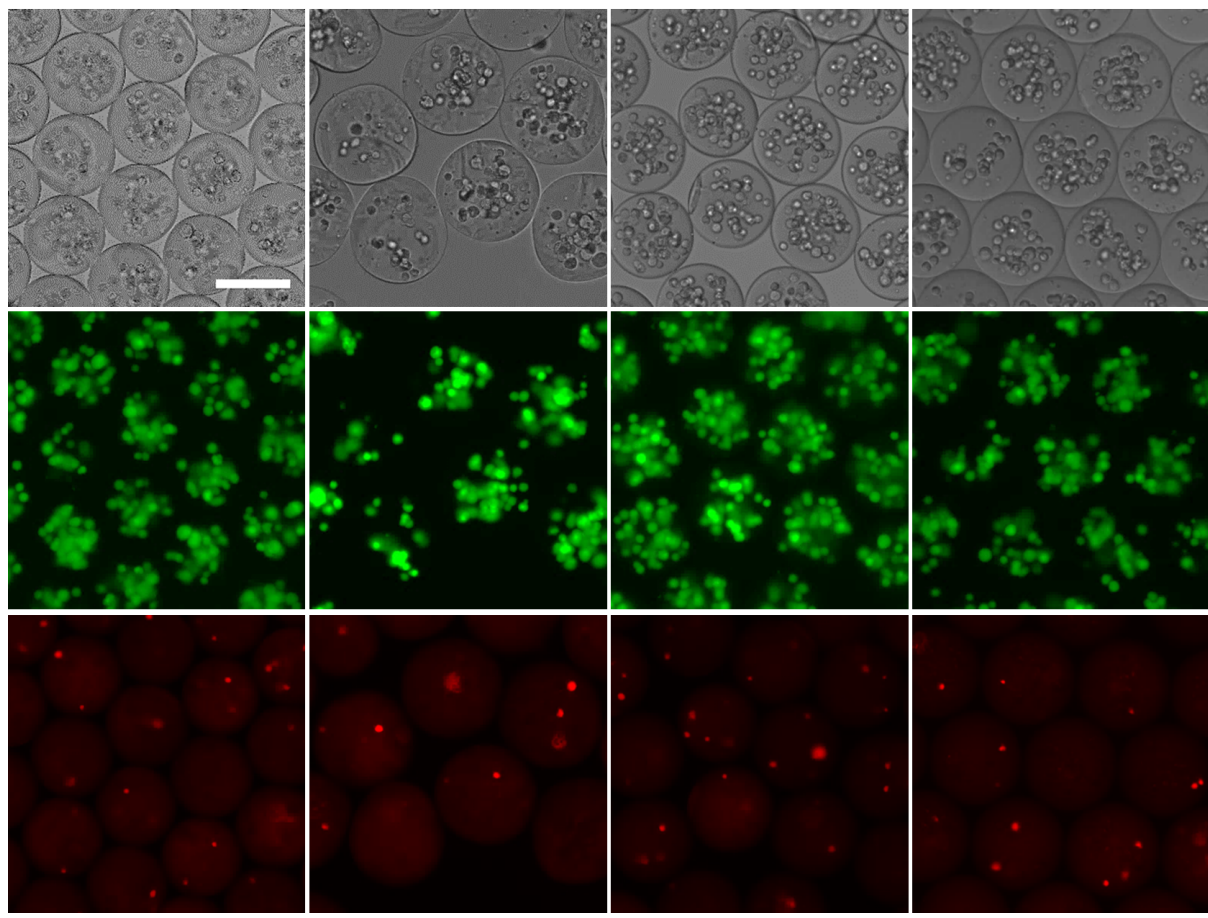

$\mathrm{B}$

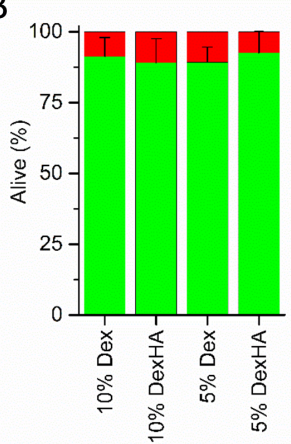

C

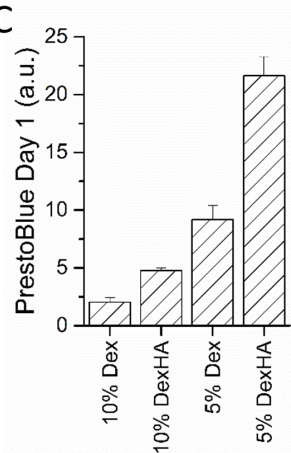

$\mathrm{D}_{450}$ Metabolic activity day 1,7 and 14

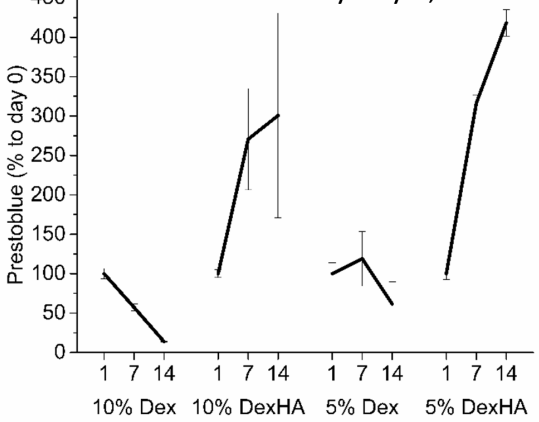

Figure 2: 2A) Overview of 4 types of microgels in phase contrast, live stain (green, calcein) and dead stain (red, ethidium homodimer) 2-4 hours after encapsulation. Scale bar represents $100 \mu \mathrm{m} .2 \mathrm{~B}$ ) Semi-Quantification of the live/dead staining, showing cell survival around $90 \%$ for all four conditions. There is no statistically significant difference between the four conditions $(P>0,05) .2 C)$ Metabolic activity at day 1 of a fixed amount of microgels reveals differences in metabolic activity already at the first day after encapsulation depending on stiffness and composition. 2D) Metabolic activity of MIN6 cells in 4 types of gels at day 1, 7 and 14, normalized to day 1. Cells encapsulated in Dex gels decrease in metabolic activity, while gels in DexHA gels increase in metabolic activity. 


\section{Morphological characterization of cell laden microgels in long term cell culture}

As shown in Figure 3A, cell laden microgels remained intact in long term cell culture experiments. Directly after encapsulation the majority of the cells were embedded as single cells (Figure 2A). Over time, cell clusters developed which increased in size (Figure $3 \mathrm{~A}$ and Figure S2). With the growth of the cell clusters, the microgels became larger in particular the 5\% DexHA composition (Figure 3B). Cell cluster formation was dependent on the stiffness of the microgels and on the presence of HA-TA. In Figure 3C, the cell clusters sizes were quantified on day 2 (Figure 3C) and day 14 (Figure 3D). The cluster size increase is shown in Figure 3E. Only in 5\% DexHA a clear increase in cluster size was observed, which was in line with the increase in diameter of the microgels (Figure 3B). 


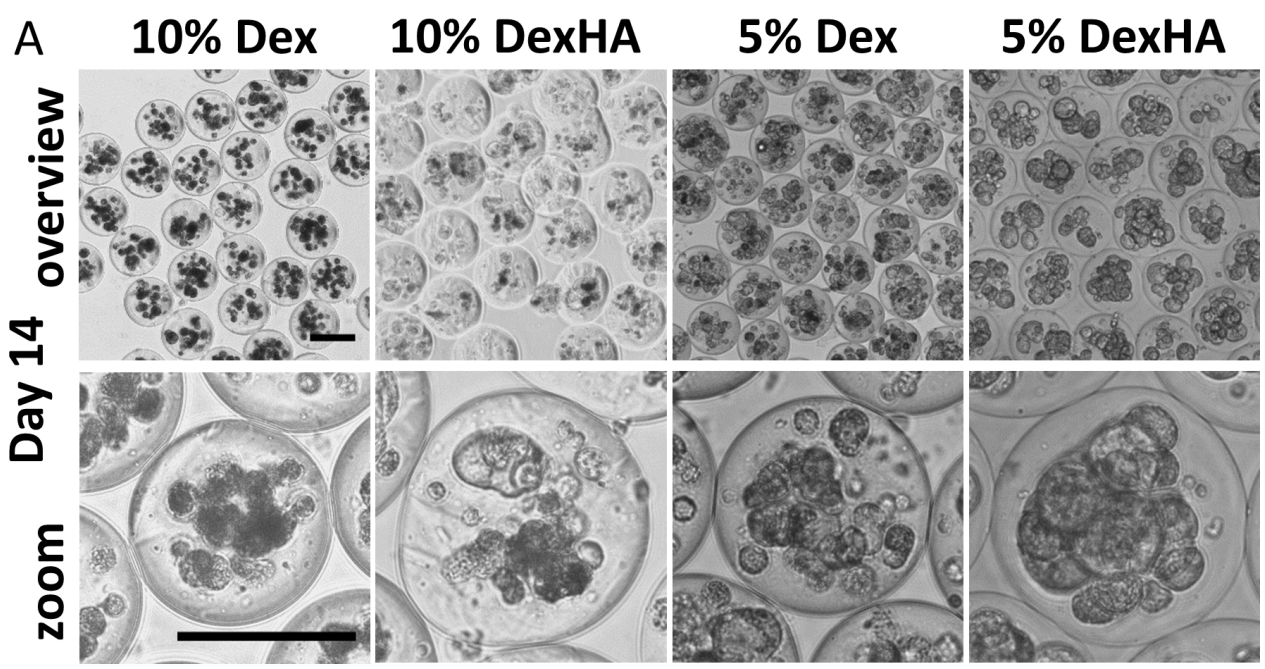

B

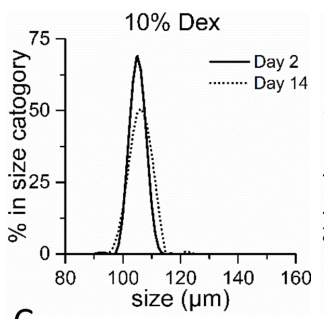

C

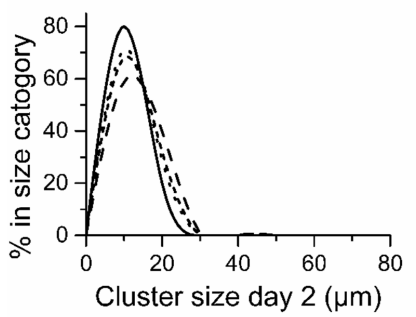

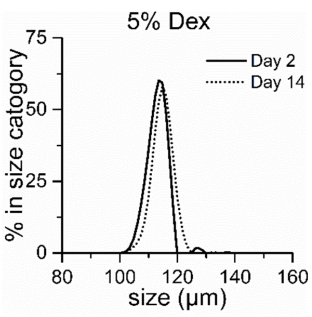

D

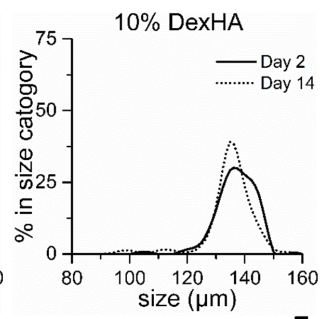

E
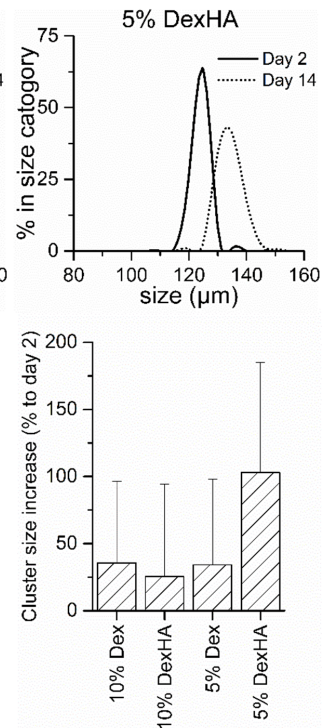

Figure 3: 3A) Overview and zoom of 4 types of gels after 14 days of culture. The cells in $10 \%$ Dex appear mostly as single cells. The cells in $10 \%$ DexHA appear as single cells, and some clusters. In $5 \%$ Dex after 14 days, there are also clusters, and single cells visible. However, in 5\% DexHA, after 14 days of culture, most gels are filled with large clusters, which increased the size of the gels. Scale bars represent $100 \mu \mathrm{m}$. 3B) Size distribution of the microgels at day 2 and day 14, confirming the size increase of 5\% DexHA gels, while the other three gel types did hardly change size. 3C-E) Cell cluster size analysis on day 2 and day 14 reveals that overtime the size of the cell clusters particularly increases in 5\% DexHA gels. 


\section{Cell proliferation analysis}

We postulated that the increase in cluster size was due to proliferation of the MIN6 cells. To test this, cell proliferation staining was performed using EDU incorporation at day 2, 7 and 14. Confocal images were taken (Figure 4A) and in these sections the total number of cells and (Figure 4B), the number of EDU positive cells were counted (Figure $4 \mathrm{C}$ ). Subsequently, the percentage of EDU positive cells / total cells was calculated (Figure 4D).

The total cell count in the 10\% Dex gels decreased over time (Figure 4B), which was in line with the downward trend in metabolic activity. For 10\% DexHA and $5 \%$ Dex, the number of cells remained fairly constant. The number of cells in 5\% DexHA dramatically increased, which was in line with the increase in metabolic activity and cluster size measurements. Note that single cells were counted as one, and large clusters as two, as it was impossible to count the exact number of cells in a larger cluster. Especially in the gels with substantial cluster formation, i.e. $10 \%$ DexHA, $5 \%$ Dex and 5\% DexHA, total cell count is an underestimation of the actual cell numbers.

On day 2, the number of EDU positive cells was comparable between all conditions (Figure 4C). At day 7 and day 14 the amount of EDU positive cells for 10\% Dex decreased to almost 0 . In contrary, in 10\% DexHA and 5\% Dex this stayed constant, while in 5\% DexHA the amount of EDU positive cells increased drastically.

When subsequently calculating the percentage of EDU positive cells (Figure 4D), this seemed fairly constant over time and between the conditions, only $10 \%$ DexTA showed a drop to almost $0 \% .5 \%$ DexHA was somewhat higher compared to $10 \%$ DexHA and 5\% Dex. 

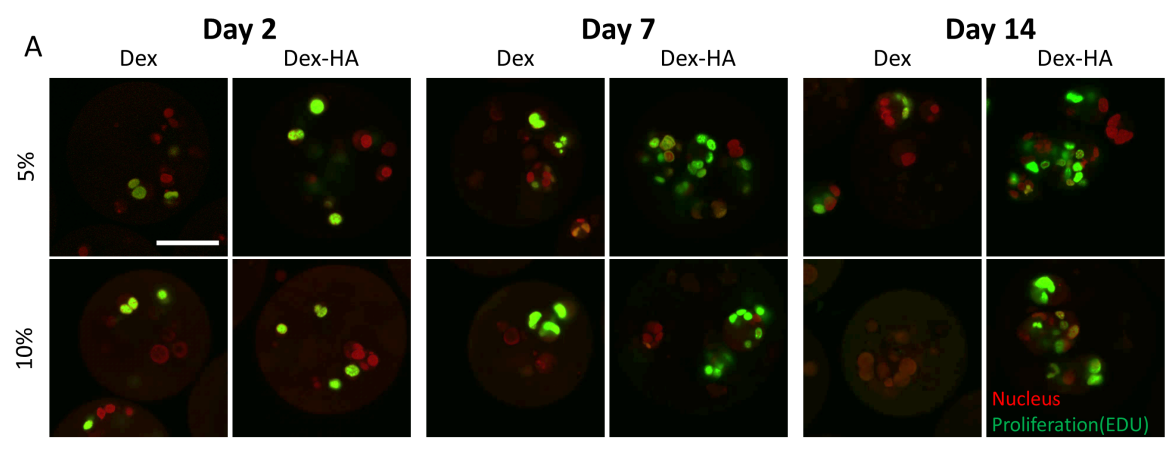

B

C

$\mathrm{D}$
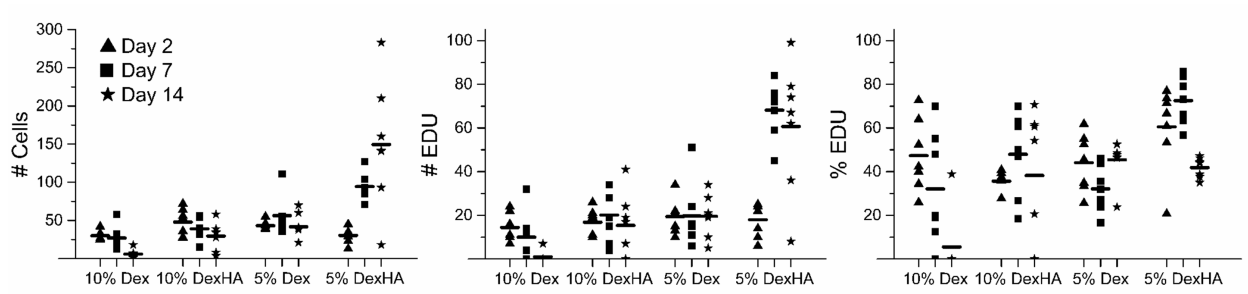

Figure 4: 4A) Confocal imaging of cell proliferation using EDU labeling at day 2, 7 and 14. Cells that proliferated after the addition of EDU (24 hours before fixation) stain green, nuclei stain red. Scale bar represents $50 \mu \mathrm{m}$. 4B-D) (semi)quantification of confocal images of EDU labeled cells, for the amount of cells, amount of edu positive cells and percentage of edu positive cells. Each point represents the average counts on 5 confocal slices. The triangle is day 2 , the square day 7 and the star day 14 . The dash represents the average.

\section{Glucose induced insulin secretion test}

We next tested whether the encapsulated MIN6 cells were able to respond to a glucose challenge at day 1 and day 7 after microgel formation (Figure 5). On day 1,10\% Dex, 10\% DexHA and 5\% Dex performed equally well. Basal levels of insulin secretion in the $5 \%$ DexHA gels were 3 fold higher. In all conditions a typical "low-high-low" profile was present. At day 1, the stimulation index (high glucose divided by 1st low glucose) was somewhat lower in gels that contained HA-TA (supplemental Figure S1).

When no HA-TA was present, insulin secretion at day 1 and day 7 was comparable. In contrast, when HA-TA was added, the basal level of insulin secretion increased two to three fold from day 1 to day 7 . When normalizing to the first low, the stimulation index in the HA-TA containing gels dropped from 3 in the $10 \%$ Dex microgels to around 2 in the 5\% DexHA gels. With respect of insulin 
secretion, particularly the presence of HA-TA rather than the gel's stiffness determined the ability to respond to glucose.
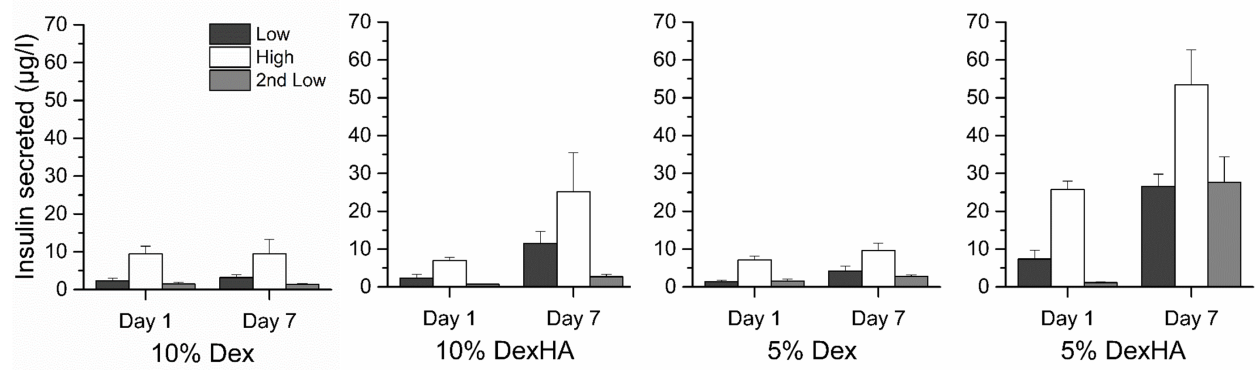

Figure 5: Glucose induced insulin secretion test on day 1 and day 7 of encapsulated MIN6 cells. All four conditions responded well to the switch from low to high glucose. Absolute values of secreted insulin are 2 to 3 times higher in 5\% DexHA compared to the other conditions at day 1 . At day 7 the $\mathrm{HA}$ containing conditions have increased basal as stimulated insulin secretion, while the responsiveness of the Dex microgels did not change.

GIIST Day 1

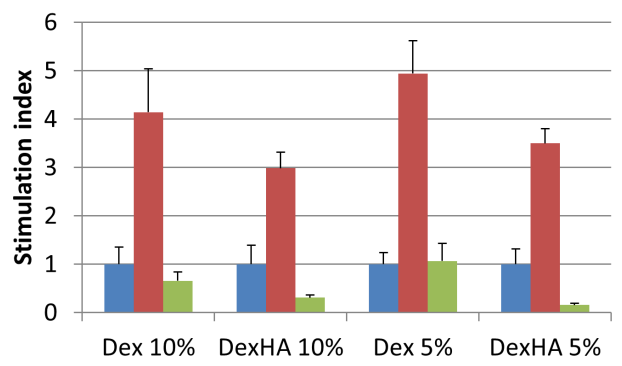

GIIST Day 7

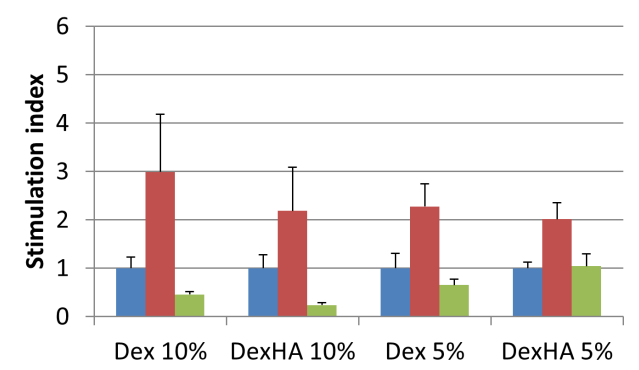

Figure S1: Glucose induced insulin secretion, normalized to the first low: "stimulation index". All conditions show a physiological insulin response, with stimulation indices at day 7 somewhat decreased compared to day 1 . 


\section{Hyaluronic acid receptor (CD44)}

Replacing $50 \%$ of DexTA with HA-TA did not affect the stiffness of the hydrogels. However, this replacement potently stimulated the metabolic activity, the proliferation, and influenced the insulin secretion profile of encapsulated cells. We postulated that this effect of HA-TA on embedded cells was due to a cell specific interaction with HA-TA mediated by the hyaluronic acid receptor CD44. To test this, we stained MIN6 cells for presence of CD44. As shown in Figure 6A, MIN6 cells stained positive for CD44, with high intensity at the cell membrane, while an isotype antibody (Figure 6B) or ablation of the first antibody (Figure 6C) only resulted in minor and diffuse background staining suggesting that MIN6 cells can sense HA via the transmembrane receptor CD44.
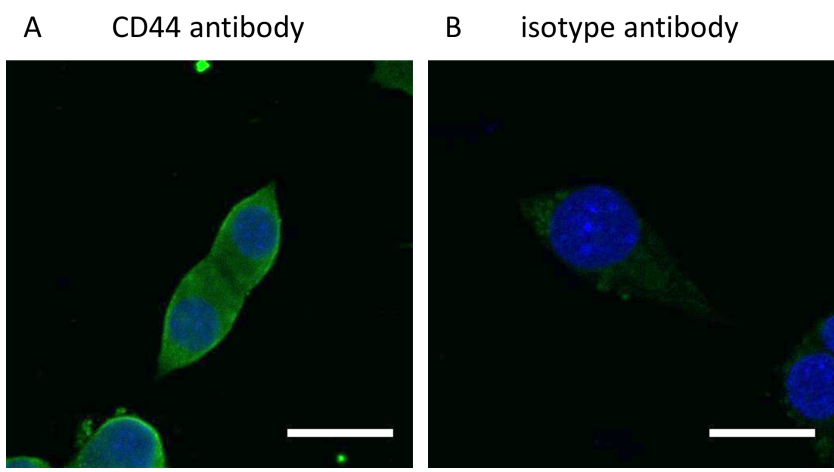

C no primary antibody

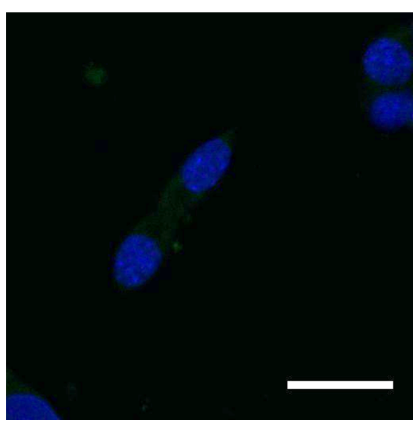

Figure 6: CD 44 staining of MIN6 cells on tissue culture plastic, using a FITC conjugated secondary antibody. A) Anti-CD44 antibody shows intense staining on the cell membrane. B) Isotype control antibody only shows minor non-specific staining in the cytoplasm. C) Negative control without primary antibody. Scale bars represent $20 \mu \mathrm{m}$.

\section{In vivo implantation}

Microgels of $10 \%$ DexHA, 5\% Dex and 5\% DexHA, and microaggregates of MIN6 cells as control, were implanted under the kidney capsule of NOD.SCID mice. After 14 days the mice were sacrificed, the kidneys explanted, fixated, paraffin embedded, sectioned and stained for the presence of microgels and insulin.

As a control micro aggregates consisting of 100 MIN6 cells each were transplanted. After 14 days, the kidneys transplanted with these microaggregates were for almost $50 \%$ invaded with MIN6 cells, as visible on both HE and 
trichrome/insulin staining (Figure 7D, H, L, P). The microaggregates of transplanted MIN6 cells demonstrated a highly aggressive and invasive phenotype. The majority of the MIN6 cells stained still slightly positive for insulin (Figure 7P). Tumor vasculature was visible (Figure 8)

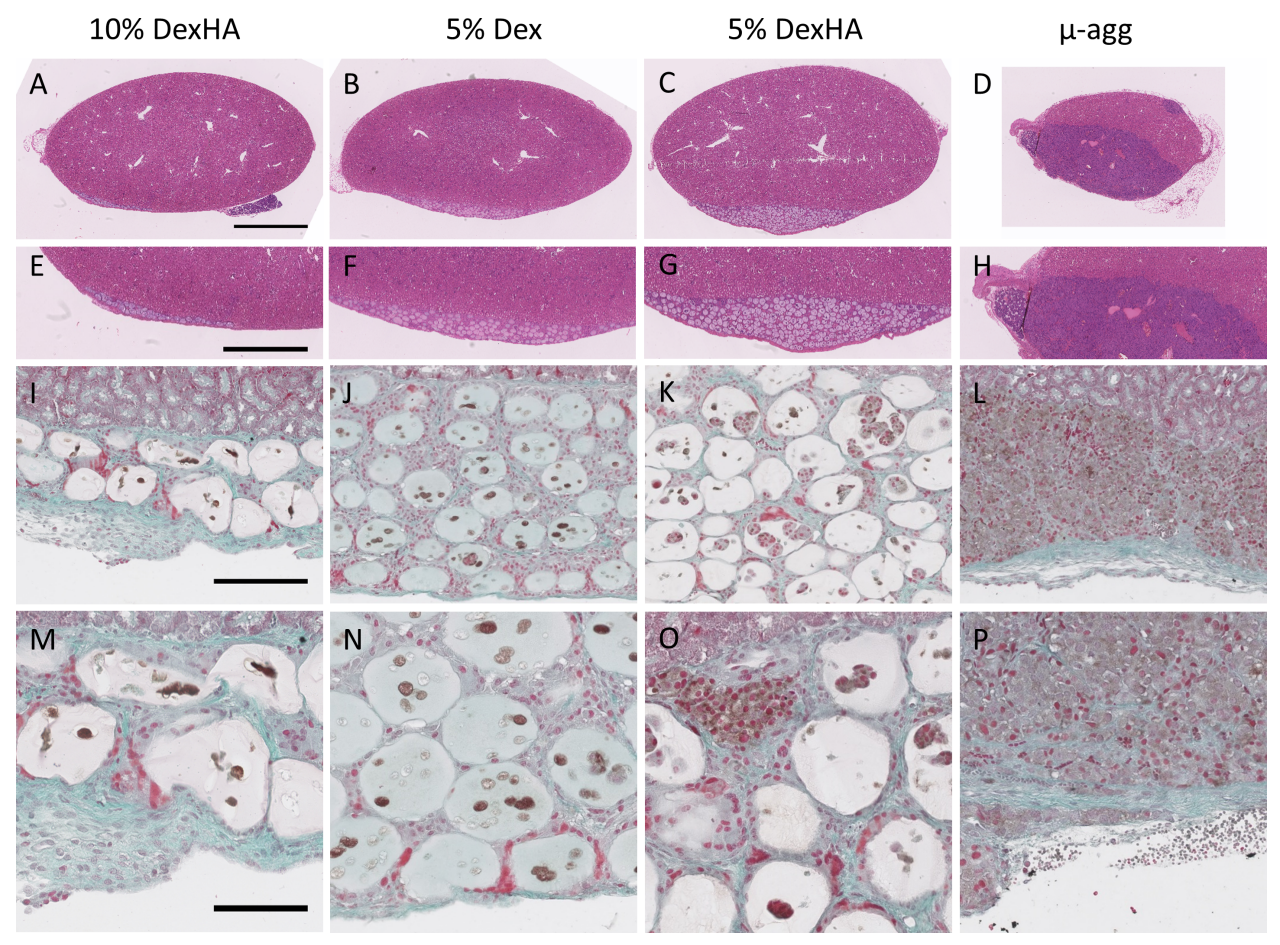

Figure 7 in vivo: Figure 7 shows microgels of 10\% DexHA, 5\% Dex, 5\% DexHA and micro aggregates implanted under the kidney capsule for 2 weeks. A-H) Hematoxylin and eosin stain, I-P) Masson's Trichrome and insulin stain. Scale bar for A-D represents $2500 \mu \mathrm{m}, \mathrm{E}-\mathrm{H}$ represents $1500 \mu \mathrm{m}, \mathrm{I}-\mathrm{L}$ represents $200 \mu \mathrm{m}$ and M-P represents $100 \mu \mathrm{m}$.

In marked contrast, encapsulation of the MIN6 cells in microgels prior to transplantation abolished this aggressive invasive behavior in gels composed of 10\% DexHA and 5\% Dex (Figure 7 I\&J). In the 5\% DexHA microgels, gel integrity was lost and MIN6 cells grew out of the microgels in a subpopulation of microgels (Figure $7 \mathrm{~K} \& O$ ). Like in vitro, in these microgels the MIN6 cells formed large clusters likely due to cell proliferation. The relatively weak stiffness of this gel composition in combination with the ability of cells and tissue to degrade HA by expressing hyaluronidase activity likely stimulated cell growth eventually 
resulting in the collapse of the microgels and cell outgrowth. Most of the cells in the 5\% DexHA microgels stained positive for insulin.

In $5 \%$ Dex microgels a few relatively small cell clusters were visible (Figure $7 \mathrm{~J}$ and $\mathrm{N}$ ). Compared to the 5\% DexHA microgels staining for insulin appeared more intense and the percentage of cells that stained positive tended to be higher (compare figure 7J, $\mathrm{N}$ and $\mathrm{O}, \mathrm{K}$ ). The shape of the 10\% DexHA 14 days after transplantation was highly variable in contrast to the other gel compositions which remained their round shape (Figure $7 \mathrm{I}$ and $\mathrm{M}$ ). Also in vitro, the shape fidelity of the $10 \%$ DexHA gels was lower compared to the other gel compositions. Cluster formation was virtually absent and most cells still stained positive for insulin (figure $7 \mathrm{I}$ and $\mathrm{M}$ )

With exception of the 5\% DexHA microgels, the 5\% Dex and 10\% DexHA gels remained intact. Furthermore, the gels remained present at the transplantation site and did not travel through the kidney. Even the gels containing HA-TA, which can be readily degraded by the enzyme hyaluronidase, which is present in most tissues, were still largely intact. The microgels were surrounded by tissue derived from the host, which also contained blood vessels in the transplants of the 5\% DexHA and 5\% Dex (Figure 8).

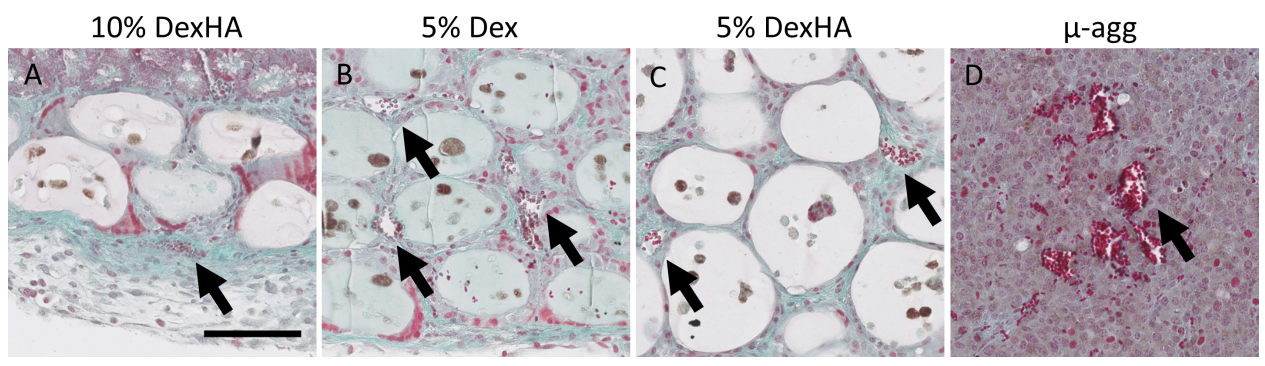

Figure 8 in vivo: Masson's Trichrome and insulin staining shows microvessels in implants, indicated by arrows. A) 10\% DexHA grafts were 2 to 3 gels (about $250 \mu \mathrm{m}$ ) thick, no obvious vessel ingrowth was visible. B\&C) Both 5\% Dex and 5\% DexHA grafts were more than $500 \mu \mathrm{m}$ thick, and had both microvessel ingrowth. D) The extensively proliferating microaggregates had typical tumor vasculature. Scale bar represents $100 \mu \mathrm{m}$. 


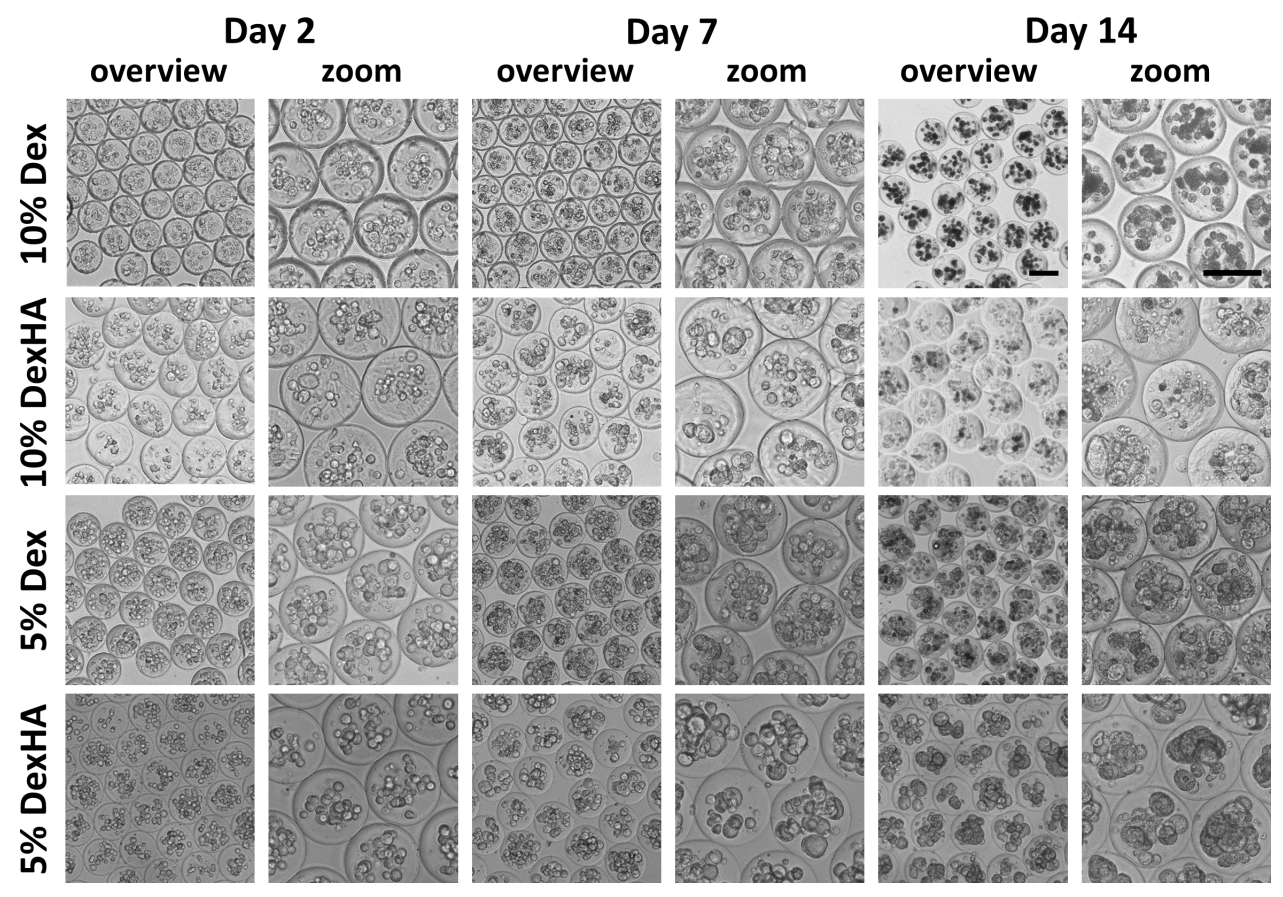

Figure S2: Overview of images taken at day 2, 7 and 14 of microgels in culture of all conditions. Scale bar represents $100 \mu \mathrm{m}$.

\section{Discussion}

iPSC derived beta cells are a promising new cell source for transplantation purposes to treat patients with type 1 diabetes. However, starting from undifferentiated iPSC current differentiation protocols cannot guarantee $100 \%$ differentiation of all cells into mature beta cells. Thus iPSC based cell therapy has the inherent risk of transplanting undifferentiated cells that may develop into teratocarcinomas ${ }^{[4,21]}$. Hence there is a need for strategies that can be used for beta cell transplantation that can impair uncontrolled proliferation of undifferentiated iPSC that remained present in the transplant.

Matrix stiffness is important for cell function and proliferation ${ }^{[18]}$. Indeed, Richardson and coworkers ${ }^{[18]}$ have shown an inverse relationship between matrix stiffness and cell proliferation. We tested the effect of two matrix stiffnesses, soft (3-3,5 kPa) and stiff ( $21 \mathrm{kPa}$ ) on proliferation of encapsulated MIN6 cells. The soft gels have a stiffness which is in the same range as the reported stiffness of the native pancreas, which depending on the report lies between $1,2 \mathrm{kPa}^{[15]}$ and 8,25 $\mathrm{kPa}{ }^{[17]}$. This set up allowed us to test our hypothesis that microgels with high 
stiffness would impair cell proliferation. However this effect might be on the expense of glucose responsiveness, since stiff microenvironments deviate substantially from the physico-mechanical properties of the native beta cell microenvironment. MIN6 cells, a cell line derived from a mouse insulinoma ${ }^{[22]}$, are a frequently used model for beta cells due to their ability to respond to a glucose challenge with insulin secretion. When transplanted in immunocompetent mice these cells display a highly aggressive and proliferative phenotype. Indeed our data show that transplantation of microaggregates of the MIN6 cells under the kidney capsule resulted in aggressive and invasive tumor growth in about $50 \%$ of the kidney in just a 2 week time period. MIN6 cells are thus a valid model for testing the development of proliferation restrictive microenvironments which do not impair glucose responsiveness and insulin secretion.

Dextran is a biocompatible polysaccharide which cannot be degraded by the body, because mammalians don't express the dextranase enzyme. A nondegradable environment seems extremely suitable to create a proliferation restricting environment, because space limitation inhibits the cell cycle ${ }^{[23]}$. A drawback of dextran is the lack of biofunctionality, as cells cannot easily attach to it or interact with it. This can be addressed by co-crosslinking of dextran with hyaluronic acid (HA). HA is a polysaccharide which is abundant in the extracellular matrix of almost all tissues in the human body ${ }^{[24]}$. HA can be degraded in the body by hyaluronidase, is pro angiogenic ${ }^{[25]}$ and interacts with cells via the CD44 membrane receptor ${ }^{[24]}$. Replacing $50 \%$ of Dex-TA by HA-TA does not affect the stiffness of the micro environment, thus this enables us to test the components independent of stiffness.

It seems likely that cells in small microgels $(<150 \mu \mathrm{m})$ suffer less than cells in large microgels from diffusion limitations. Decreasing the size of capsules is relevant since functional beta cells need to be in close contact with vasculature. In fact, while only constituting 1-2 percent of pancreas mass, Islets receive 10 percent of the pancreatic blood flow ${ }^{[26]}$. Again, the smaller the unit the better, because of decreased distance between beta cells and vessels. Especially survival of the hypoxia during the first days after transplantation is critical. The microgels allowed for easy cell observation, assays and transplantation using established 
techniques. We encapsulated MIN6 cells in these microgels and studied them during 2 weeks of culture and during 2 weeks of in vivo implantation.

First we studied the cell encapsulating microgels in vitro. Cell survival after encapsulation was high and irrespective of the polymer composition. However, metabolic activity measurement after 1 day already revealed large differences between polymer compositions. We found an effect of the stiffness and of the presence of HA-TA, probably these are two separate effects, as both lower stiffness and addition of HA approximately double the metabolic activity. Combined resulted the low stiffness and $\mathrm{HA}$ in a roughly eight times higher metabolic activity in 5\% DexHA compared to 10\% Dex. Subsequently, gels with HA-TA sustained, and even increased, the metabolic activity of the encapsulated cells over time, while without HA-TA the metabolic activity decreased. For the soft $5 \%$ Dex the metabolic activity first went up, but soft gel alone is not enough to keep the MIN6 cells metabolically active. We subsequently showed that the increase in metabolic activity over time is (partially) due to cell proliferation. Proliferating cells don't migrate through the microgel, but they form cell clusters. Note that individual cells can't be counted in a cluster due to dense packing, resulting in an underestimation in the cell counts, and thus seemingly mismatch with the metabolic activity data. The growth of these clusters was visible in all conditions, but most pronounced in 5\% DexHA. This growth of cell clusters made the microgels increase in size in this condition.

Another important evaluation aspect was whether the MIN6 cells were able to respond to a glucose challenge. As in all conditions the same amount of cells were encapsulated, we expected comparable amounts of insulin at day 1. This was the case, except for $5 \%$ DexHA, in which the secretion level was 3 times higher compared to the other conditions. This could be attributed to the combination effect of low stiffness and HA-TA, which also led to a higher metabolic activity. After a week of culture the amount of secreted insulin increased when HA-TA was present, in line with the metabolic activity. We hypothesize that this is due to the proliferation in these gels. Remarkably, when HA-TA was added, the stimulation index dropped one point, especially on day 1. There seems no relationship between glucose responsiveness and stiffness of the microenvironment, stiffness only influences the insulin secretion by increased proliferation. However, the use of HA-TA in our system shows that it would be 
possible to optimize the glucose responsiveness of the microenvironment, irrespective of the stiffness, by tuning the matrix by co-crosslinking with other extracellular matrix (ECM) molecules that have a positive effect on beta cell function, like laminin ${ }^{[27]}$.

In order to study the behavior of the cells in the microgels in vivo we implanted the cell encapsulating microgels in NOD.SCID mice. As we saw massive cell dead in the $10 \%$ Dex in vitro, we did not include this condition. As a control we used non-encapsulated MIN6 microaggregates as a model to check the invasiveness of the cells. Gels and aggregates were implanted under the kidney capsule, and histologically analyzed after 2 weeks in vivo. The highly invasive nature of the MIN6 cells was confirmed by the MIN6 microaggregates, which invaded approximately half of the kidney with slightly insulin positive tumor tissue in just two weeks. In the 5\% DexHA gels the MIN6 cells formed aggregates, and some outgrowth of insulin positive cells was visible. The gel clearly confined the cells to certain extent, but not enough to prevent uncontrolled cell proliferation similar like in vitro. In the contrary, in both 10\% DexHA and 5\% Dex the cells stained still clearly positive for insulin, and no cell outgrowth was visible. In the 5\% Dex microvessels surrounding the microgels of the transplant were clearly visible, in $10 \%$ DexHA the grafts were too small to find vessels in the graft itself. We hypothesize that the quick vascularization is due to the round gels, because packing doesn't result in a dense pack which is hard for vessels to grown in.

In conclusion, it is possible to make a proliferation restrictive environment using microgels, while sustaining glucose responsiveness. The cells survive, also in vivo, and the presence of vessels indicates that these microgels could be part of a potential successful strategy for beta cell transplantation purposes. Compared to existing strategies like macro encapsulation or alginate encapsulation, these small microgels are less prone to diffusion limitation and easily tunable using tyramine conjugated materials or growth factors. Their round shape leaves sufficient space for vessel in growth and rapid vascularization of the transplant. Engineering proliferation restrictive microenvironments could potentially be part of iPSC based transplantations procedures to prevent teratocarcinoma formation. 


\section{Acknowledgements}

The authors gratefully thank Johan Bomer of the BIOS Lab-on-a-chip group of University of Twente for his expertise in micromolding, and Jan Hendriks of the Developmental BioEngineering group of University of Twente for his assistance with Matlab analysis.

\section{References:}

[1] J. B. Buse, S. Caprio, W. T. Cefalu, A. Ceriello, S. Del Prato, S. E. Inzucchi, S. McLaughlin, G. L. Phillips, R. P. Robertson, F. Rubino, R. Kahn, and M. S. Kirkman, "How do we define cure of diabetes?", Diabetes Care, vol. 32, no. 11, pp. 2133-2135, 2009.

[2] G. M. Danovitch, "Immunosuppressive medications and protocols", in Handbook of Kidney Transplantatio, 2005, pp. 72-134.

[3] F. W. Pagliuca, J. R. Millman, M. Gürtler, M. Segel, A. Van Dervort, J. H. Ryu, Q. P. Peterson, D. Greiner, and D. A. Melton, "Generation of Functional Human Pancreatic $\beta$ Cells In Vitro", Cell, vol. 159, no. 2, pp. 428-439, 2014.

[4] S. G. Hong, T. Winkler, C. Wu, V. Guo, S. Pittaluga, A. Nicolae, R. E. Donahue, M. E. Metzger, S. D. Price, N. Uchida, S. A. Kuznetsov, T. Kilts, L. Li, P. G. Robey, and C. E. Dunbar, "Path to the clinic: assessment of iPSCbased cell therapies in vivo in a nonhuman primate model.", Cell reports, vol. 7, no. 4, pp. 1298-309, 2014.

[5] M. Kumagai-Braesch, S. Jacobson, H. Mori, X. Jia, T. Takahashi, A. Wernerson, M. Flodström-Tullberg, and A. Tibell, "The theracyte ${ }^{\mathrm{TM}}$ device protects against islet allograft rejection in immunized hosts", Cell Transplantation, vol. 22, no. 7, pp. 1137-1146, 2013.

[6] K. Skrzypek, M. Groot Nibbelink, M. Coronel, I. Labrada, C. Stabler, J. Van Lente, M. Karperien, A. van Apeldoorn, and D. Stamatialis, "Evaluation of microwell PES/PVP membranes for islet encapsulation: a novel strategy for immune protection", In Preparation, 2016.

[7] E. S. O'Sullivan, A. Vegas, D. G. Anderson, and G. C. Weir, "Islets transplanted in immunoisolation devices: $A$ review of the progress and the challenges that remain", Endocrine Reviews, vol. 32, no. 6, pp. 827-844, 2011.

[8] J. Beck, R. Angus, B. Madsen, D. Britt, B. Vernon, and K. T. Nguyen, "Islet encapsulation: strategies to enhance islet cell functions.", Tissue engineering, vol. 13, no. 3, pp. 589-599, 2007.

[9] K. Y. Lee and D. J. Mooney, "Alginate: Properties and biomedical applications", Progress in Polymer Science, vol. 37, no. 1, pp. 106-126, 
2012.

[10] J. W. H. Wennink, K. Niederer, A. I. Bochyńska, L. S. Moreira Teixeira, M. Karperien, J. Feijen, and P. J. Dijkstra, "Injectable Hydrogels by Enzymatic Co-Crosslinking of Dextran and Hyaluronic Acid Tyramine Conjugates", Macromolecular Symposia, vol. 309-310, no. 1, pp. 213-221, 2011.

[11] R. Jin, L. S. Moreira Teixeira, P. J. Dijkstra, C. a. van Blitterswijk, M. Karperien, and J. Feijen, "Chondrogenesis in injectable enzymatically crosslinked heparin/dextran hydrogels", Journal of Controlled Release, vol. 152, no. 1, pp. 186-195, 2011.

[12] R. Jin, C. Hiemstra, Z. Zhong, and J. Feijen, "Enzyme-mediated fast in situ formation of hydrogels from dextran-tyramine conjugates", Biomaterials, vol. 28, no. 18, pp. 2791-2800, 2007.

[13] S. Henke, J. Leijten, E. Kemna, M. Neubauer, A. Fery, A. van den Berg, A. van Apeldoorn, and M. Karperien, "Enzymatic Crosslinking of Polymer Conjugates is Superior over Ionic or UV Crosslinking for the On-Chip Production of Cell-Laden Microgels", Macromolecular Bioscience, vol. 16, no. 10, pp. 1524-1532, 2016.

[14] C. J. Rall and A. K. Rustgi, "CD44 isoform expression in primary and metastatic pancreatic adenocarcinoma", Cancer Res, vol. 55, no. 9, pp. 1831-5, 1995.

[15] S.-K. Goh, S. Bertera, P. Olsen, J. E. Candiello, W. Halfter, G. Uechi, M. Balasubramani, S. A. Johnson, B. M. Sicari, E. Kollar, S. F. Badylak, and I. Banerjee, "Perfusion-decellularized pancreas as a natural 3D scaffold for pancreatic tissue and whole organ engineering." , Biomaterials, vol. 34, no. 28, pp. 6760-72, 2013.

[16] M. Sugimoto, S. Takahashi, M. Kojima, N. Gotohda, Y. Kato, S. Kawano, A. Ochiai, and M. Konishi, "What is the nature of pancreatic consistency? Assessment of the elastic modulus of the pancreas and comparison with tactile sensation, histology, and occurrence of postoperative pancreatic fistula after pancreaticoduodenectomy.", Surgery, vol. 156, no. 5, pp. 1204-11, 2014.

[17] Z. Dong, U. C. Wejinya, Y. Zhu, and K. Ye, "Force measurement study of engineered collagen-chitosan scaffold using Atomic Force Microscopy", in Nano/Molecular Medicine and Engineering (NANOMED), 2010 IEEE 4th International Conference on, 2010, pp. 184-187.

[18] T. Richardson, S. Barner, J. Candiello, P. N. Kumta, and I. Banerjee, "Capsule stiffness regulates the efficiency of pancreatic differentiation of human embryonic stem cells", Acta Biomaterialia, 2016.

[19] J. Hilderink, S. Spijker, F. Carlotti, L. Lange, M. Engelse, C. van Blitterswijk, E. de Koning, M. Karperien, and A. van Apeldoorn, "Controlled aggregation of primary human pancreatic islet cells leads to glucose-responsive 
pseudoislets comparable to native islets.", Journal of cellular and molecular medicine, vol. 19, no. 8, pp. 1836-46, 2015.

[20] L. S. Moreira Teixeira, J. C. H. Leijten, J. Sobral, R. Jin, a. a. van Apeldoorn, J. Feijen, C. van Blitterswijk, P. J. Dijkstra, and M. Karperien, "High throughput generated micro-aggregates of chondrocytes stimulate cartilage formation in vitro and in vivo", Eur Cell Mater, vol. 23, pp. 387399, 2012.

[21] J. J. Cunningham, T. M. Ulbright, M. F. Pera, and L. H. J. Looijenga, "Lessons from human teratomas to guide development of safe stem cell therapies", Nature Biotechnology, vol. 30, no. 9, pp. 849-857, 2012.

[22] J. Miyazaki, K. Araki, E. Yamato, H. Ikegami, T. Asano, Y. Shibasaki, Y. Oka, and K. Yamamura, "Establishment of a pancreatic beta cell line that retains glucose-inducible insulin secretion: special reference to expression of glucose transporter isoforms", Endocrinology, vol. 127, no. 1, pp. 126-132, 1990.

[23] C. Cadart, E. Zlotek-Zlotkiewicz, M. Le Berre, M. Piel, and H. K. Matthews, "Exploring the function of cell shape and size during mitosis", Developmental Cell, vol. 29, no. 2, pp. 159-169, 2014.

[24] J.-W. Kuo and G. D. Prestwich, Hyaluronic Acid. Elsevier Ltd., 2011.

[25] M. Xu, X., Jha, A, Harrington, DA., Farach-Carson, "Hyaluronic Acid - Based Hydrogel: from a Natural Polysaccharide to Complex Networks", Soft Matter, vol. 8, no. 12, pp. 3280-3294, 2012.

[26] N. Yaginuma, T. Takahashi, K. Saito, and M. Kyoguku, "The microvasculature of the human pancreas and its relation to Langerhans islets and lobules.", in The Pancreas: Biology, Pathobiology, and Disease, Second Edition, 1993, p. 760.

[27] L. M. Weber, K. N. Hayda, and K. S. Anseth, "Cell-matrix interactions improve beta-cell survival and insulin secretion in three-dimensional culture", Tissue Eng Part A, vol. 14, no. 12, pp. 1959-1968, 2008. 


\section{Chapter 5}

\section{Biofabrication of core-shell hydrogels as microbioreactors for controlled cell aggregation}

Sieger Henke*, Tom Kamperman*, Jacqueline Plass, Rong Wang, Pieter J. Dijkstra, Jeroen Leijten, Marcel Karperien

* SH and TK are shared first author 


\section{Abstract}

In this study we present an easy to use technology platform for the production of cell-laden core-shell microgels consisting of polymer-tyramine conjugates that crosslink in a three-dimensional macromolecular network using a cell friendly peroxidase mediated reaction initiated by low concentrations of $\mathrm{H}_{2} \mathrm{O}_{2}$. First droplet trains in oil of cells and polymer conjugates are formed, which are subsequently traversed in a silicone tubing through a $\mathrm{H}_{2} \mathrm{O}_{2}$ water bath. By diffusion of $\mathrm{H}_{2} \mathrm{O}_{2}$ through the silicone tubing and oil phase, outside-in crosslinking of the polymer conjugates is achieved. The amount of crosslinking in the microgels strictly depended on the retention time of the droplets in the silicone tubing, and was easily controlled by the flow speed of the oil phase. At low flow speed crosslinking was achieved in both the core and the shell. With increasing flow speed crosslinking in the core was reduced and hollow microgels were obtained. Flow speed also controlled the crosslinking density in the shell of the microgels and inversely impacted on diffusion of dextran-FITC conjugates in a size range of $2000 \mathrm{kDa}$ to $20 \mathrm{kDa}$.

The method for production of cell-laden microgels was cell friendly in short term and long term follow up with more than $90 \%$ survival of cells 4 hours after encapsulation even at low flow speed and thus relatively high exposure to $\mathrm{H}_{2} \mathrm{O}_{2}$. When loaded with approximately 120 human primary mesenchymal stem cells or 70 MIN6 beta cells, cell aggregation in the microgels could be easily controlled by the crosslinking density in the core of the microgels. In long term cell culture, cell aggregation of MSCS or MIN6 cells was absent or delayed, respectively in gels with a crosslinked core. In the hollow microgel configuration both cell types started to aggregate in a large cluster within 24 hours. This behavior was also observed in an ex vivo organ culture model.

In conclusion, our platform could be used for the high throughput generation of cell-laden hollow microgels that could serve as microbioreactors to control cell aggregation both in vitro and upon injection in vivo. 


\section{Introduction}

There is a strong need for controlled cell aggregation in a variety of biological processes such as the initiation of stem cell differentiation or the formation of three-dimensional microtissues. Controlled aggregation of small amounts of cells has been challenging, as cell aggregation in a non-adherent plate is based on a random process, resulting in aggregates of largely differing sizes ${ }^{[1]}$. Better control of the aggregation process is possible in multiwell plates ${ }^{[2]}$, tubes ${ }^{[3]}$, or in hanging drop assays ${ }^{[3]}$, but these procedures are labor intensive and often require large quantities of cells. With the introduction of micro technology for the production of microwells, it became possible to aggregate small amounts of cells in a controlled manner ${ }^{[4-6]}$. In these approaches, cells are aggregated in a non-adherent, but also non-stimulating environment based on the tendency of cells to cluster via homotypic interactions between cell adhesion molecules expressed at the cell membrane ${ }^{[7]}$. Micro aggregates consisting of a few up to several hundreds of cells can be easily obtained. However, long term culture of these micro aggregates in microwell platforms is difficult, as aggregates, disturbed by medium change or handling of the plates, could stick together. Furthermore, when introduced in an environment which facilitates attachment of cells to extracellular matrix molecules or tissue culture plastic, these micro aggregates tend to spread out or disintegrate.

Recently, microfluidic technology enabled controlled aggregation of small numbers of cells in microtraps, as shown for example by Wu et al. ${ }^{[8]}$. This allowed them to study individual aggregates of cancer cells in high throughput for drug discovery. However, this approach is limited by the amount of on-chip traps. Alternatively, droplet microfluidics could be used for the on-chip production of cell-laden microgels ${ }^{[9,10]}$. These microgels can be produced in at least 2 distinct configurations; cell-laden solid microgels which generally would impair cell movement and interaction ${ }^{[9]}$ or core-shell configurations in which cells in the core can freely move ${ }^{[11-14]}$. To produce hollow microcapsules, solid alginate microgels can be used as a template in combination with Poly-L-Lysine technology or other materials ${ }^{[15,16]}$. This technology is, however, usually limited to the biomaterial alginate and requires a multi-step process of encapsulating and coating. For example Sakai and coworkers made hollow microgels in a 3-step process, starting with the formation of a solid core, the subsequent coating with a shell, followed 
by enzymatic degradation of the core ${ }^{[17]}$. Ashida and coworkers made hollow microgels in a single-step process using a tyramine conjugated alginate that can be crosslinked with the enzyme horseradish peroxidase (HRP) in a reaction that is strictly dependent on the co-activator $\mathrm{H}_{2} \mathrm{O}_{2}{ }^{[12]}$. Using droplet microfluidics polymer conjugate, HRP and the enzyme catalase containing droplets were produced. The latter enzyme neutralizes $\mathrm{H}_{2} \mathrm{O}_{2}$. By presenting $\mathrm{H}_{2} \mathrm{O}_{2}$ mixed in the oil phase to these droplets outside-in crosslinking of the polymer conjugates is achieved, generating core-shell microgels. This system is, however, difficult to tune and control, because it depends on the balance between two enzymes, which have different optimal working conditions, and the unstable emulsion of $\mathrm{H}_{2} \mathrm{O}_{2}$ in oil.

The most commonly reported methods to fabricate core-shell hydrogels require access to micro or nanofabrication technology to produce microfluidic devices, which is not widely available, hampering wide spread introduction in biological research. In this study we present a relatively easy setup to produce tunable core-shell microgels of natural and synthetic polymer conjugates by adapting the method reported by Ashida et al. ${ }^{[12]}$. Our method also relies on HRP mediated crosslinking of polymer-tyramine conjugates. This reaction is fast, usually within a few seconds, and strictly depends on $\mathrm{H}_{2} \mathrm{O}_{2}$ as activator. The small size of this molecule enables its diffusion through a wide variety of materials including oil and silicone tubing. First, we form a droplet train containing the polymer conjugate and the enzyme in oil in a silicon tube, and the subsequent submerging of the tubing in a $\mathrm{H}_{2} \mathrm{O}_{2}$ solution. By diffusion of $\mathrm{H}_{2} \mathrm{O}_{2}$ through the tubing and the oil phase into the droplets, outside-in crosslinking of the polymer conjugates is induced. The crosslinking density as well as the shell thickness is controlled by the retention time of the droplets in the silicone tubing traversing through the $\mathrm{H}_{2} \mathrm{O}_{2}$ water bath. Using this technology we demonstrate fast and robust engineering of specialized micro bioreactors for controlled cell aggregation and their subsequent biological evaluation both in vitro and ex vivo. 


\section{Materials and Methods}

\section{Cell culture}

MIN6B1 cells were cultured in DMEM (Invitrogen), supplemented with 10 \% FBS (Sigma-Aldrich), $71 \mu \mathrm{M}$ 2-mercaptoethanol (Invitrogen) and $100 \mathrm{U} / \mathrm{ml}$ penicillin and $100 \mu \mathrm{g} / \mathrm{ml}$ streptomycin (Invitrogen) and used from passage 30 to 40. The use of patient material was approved by the local ethical committee of the Medisch Spectrum Twente, and informed written consent was obtained for all samples. Human mesenchymal stem cells (hMSCs) were isolated from bone marrow as described previously ${ }^{[18]}$. The cells were cultured in Alfa-MEM (Invitrogen), supplemented with 10 \% FBS (Sigma-Aldrich), $2 \mathrm{mM} \mathrm{L-glutamine}$ (Invitrogen), 0,2 $\mathrm{mM}$ ascorbic acid (Sigma-Aldrich), $1 \mathrm{ng} / \mathrm{ml}$ basic fibroblast growth factor (ISOKine bFGF, Neuromics), and $100 \mathrm{U} / \mathrm{ml}$ penicillin and $100 \mathrm{\mu g} / \mathrm{ml}$ streptomycin (Invitrogen). Cells were kept in a humidified environment at $37^{\circ}$ degrees Celsius with $5 \% \mathrm{CO}_{2}$ and used in passage 3 .

\section{Polymer conjugates}

Dextran-Tyramine (18460 g/mol (15000 - $30000 \mathrm{~g} / \mathrm{mol}$, Sigma-Aldrich)), Hyaluronic Acid-Tyramine (16000 g/mol (15000 - $30000 \mathrm{~g} / \mathrm{mol}$, Contipro)) (Figure 1B), and 8-arm poly(ethylene glycol)-Tyramine (8PEG, $20000 \mathrm{~g} / \mathrm{mol}$, Jenkem Technology) (Figure 1C) conjugates were prepared as described previously ${ }^{[19,20]}$. Dex-TA was used with a DS (Degree of Substitution, the number of tyramine residues per 100 disacharide blocks) of 15, HA-TA with a DS of 2, PEG-TA as PEG$\mathrm{TA}_{5}-\mathrm{OH}_{3}$. DexHA gels contained $5 \mathrm{wt} / \mathrm{v} \%$ Dex-TA and $1 \mathrm{wt} / \mathrm{v} \% \mathrm{HA}-\mathrm{TA}$ and $40 \mathrm{U} / \mathrm{ml}$ HRP (Sigma-Aldrich). PEG gels contained $10 \mathrm{wt} / \mathrm{v} \%$ PEG-TA and $80 \mathrm{U} / \mathrm{ml} \mathrm{HRP.}$

\section{Microgel formation}

The microfluidic device consisted off a piece of perspex (approximately $20 \times 20 \times 10 \mathrm{~mm}$ ) with holes drilled for inlets and outlet. Fluids were infused by a syringe pump. Droplets were formed by dripping the aqueous phase (polymertyramine conjugates with horse radish peroxidase, with or without cells) in the oil phase (Hexadecane with $1 \%$ Span 80 ) into a capillary inserted via the outlet of the perspex (fused silica, outer diameter (OD) $360 \mu \mathrm{m}$, inner diameter (ID) 200 $\mu \mathrm{m})$. The tip of the capillary was polished at an angle of approximately 45 degrees and coated with Aquapel (Vulcavite) to ensure hydrophobicity. A delay tubing 
(310 $\mu \mathrm{m}$ ID, $640 \mu \mathrm{m}$ OD, $150 \mu \mathrm{m}$ WD, Helix Medical) was connected to the capillary, and immersed over a length of approximately $21,5 \mathrm{~cm}$ in $30 \%$ hydrogen peroxide (Sigma-Aldrich). The outside-in crosslinking of the droplets was induced by diffusion of hydrogen peroxide trough the tubing and oil into the polymer conjugates and HRP containing droplets. The residence time in the tubing was altered by increasing the total flow speed through the system, while keeping the ratio water:oil constant at 1:10 (except for $16 \mu \mathrm{l} / \mathrm{min} \mathrm{PEG}$, which has a water:oil ratio of 1:15). At a flow speed of $44 \mu \mathrm{l} / \mathrm{min}$, the residence time in the $\mathrm{H}_{2} \mathrm{O}_{2}$ diffusion bath was approximately 22 seconds. The gels were collected in oil, and the emulsion was broken by washing 3 times with hexadecane and subsequently once with culture medium. Cell containing gels were cultured in multiwell plates (Nunc).

A Tyramine conjugate
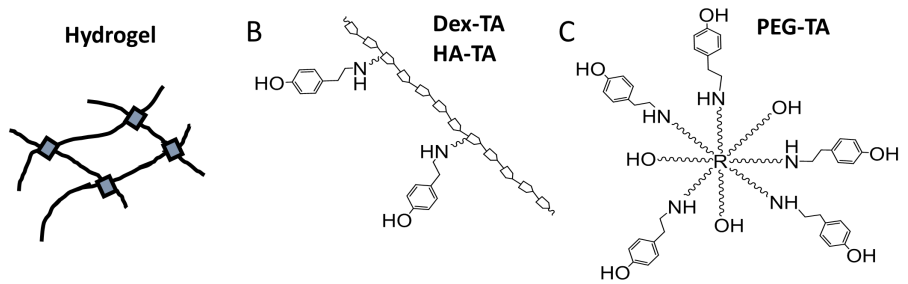

D 3D coaxial flow focusing droplet generator

Outside-in crosslinking Tyramine conjugate
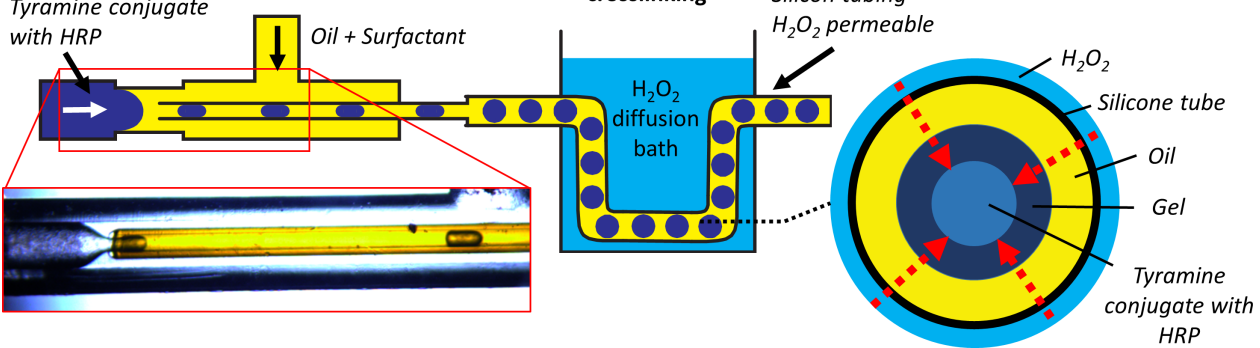

Figure 1: A) Schematic representation of crosslinking of tyramine conjugates into a macromolecular hydrogel network. B) Schematic representation of dextran and hyaluronic acid-Tyramine conjugates C) Schematic representation of the crosslinking of 8-arm star-shaped PEG-Tyramine conjugates. D) Schematic overview of the device with the droplet generator on the left (and a picture on the bottom), the $\mathrm{H}_{2} \mathrm{O}_{2}$ diffusion bath in the middle, and a cross-sectional view of the immersed diffusion tubing on the right. 


\section{Live/dead analysis}

Cells were stained using calcein AM and ethidium homodimer-2, according to manufacturer's protocol (Invitrogen) and imaged using a digital florescence microscope (EVOS, Invitrogen).

\section{Microgel visualization and analysis}

Ethidium homodimer (30 $\mathrm{MM}$, Invitrogen) was used to visualize the crosslinked polymer for confocal microscopy (Nikon A1 confocal). A crosssectional view was generated to analyze the shell.

\section{Permeability}

The permeability of the gels was tested using FITC-conjugated dextrans with molecular weights of 20, 40, 70, 150, 500 and 2000 kDa (Sigma-Aldrich) and immunoglobulin G (IgG, 150 kDa, Sigma-Aldrich). Confocal cross-sections were made and the fluorescence intensity in the center of the gel was compared with the fluorescence intensity of the background using a custom made Matlab script. A minimum of 50 gels per condition were analyzed.

\section{Aggregation ex vivo}

In order to test the aggregation in a model close to the in vivo situation, we implanted microgels by simple injection in the explanted kidney and peritoneal fat of a freshly sacrificed rat (Figure 7)(Whistar, 20 days old). The explants were subsequently cultured for 24 hours in normal MIN6 culture medium.

\section{Histology}

Samples were formalin fixed (10\% neutrally buffered formalin, 24 hours) and routinely processed for histology and embedded in paraffin. $5 \mu \mathrm{m}$ thick sections were cut, applied on microscopy slides (superfrost plus, Menzel), and stained with Hematoxylin and Eosin. Images of slides were made using a slide scanner (Nanozoomer, Hammamatsu).

\section{Results}

Using the 3D microfluidic system described in Figure 1D, we were able to produce stable hollow core-shell microgels of around $250 \mu \mathrm{m}$ in size, with the 
shell thickness depending on the material and residence time in the $\mathrm{H}_{2} \mathrm{O}_{2}$ diffusion tubing. Droplets were formed at a frequency of approximately $100-900$ droplets per minute, depending on the flow speed. In order to show the versatility of the diffusion technique, we used two different materials: a mix of natural polymer conjugates consisting of $5 \mathrm{wt} / \mathrm{v} \%$ Dextran-Tyramine and $1 \mathrm{wt} / \mathrm{v} \%$ Hyaluronic Acid-Tyramine (DexHA), and a synthetic 8-arm Poly Ethylene GlycolTA (PEG) conjugate. These polymer conjugates crosslink in a mild enzymatic reaction initiated by low concentrations of $\mathrm{H}_{2} \mathrm{O}_{2}$. Both polymers have their specific properties and applications. Using fluorescence as readout, crosslinking density of the polymeric networks can be determined as previously shown ${ }^{[21]}$.

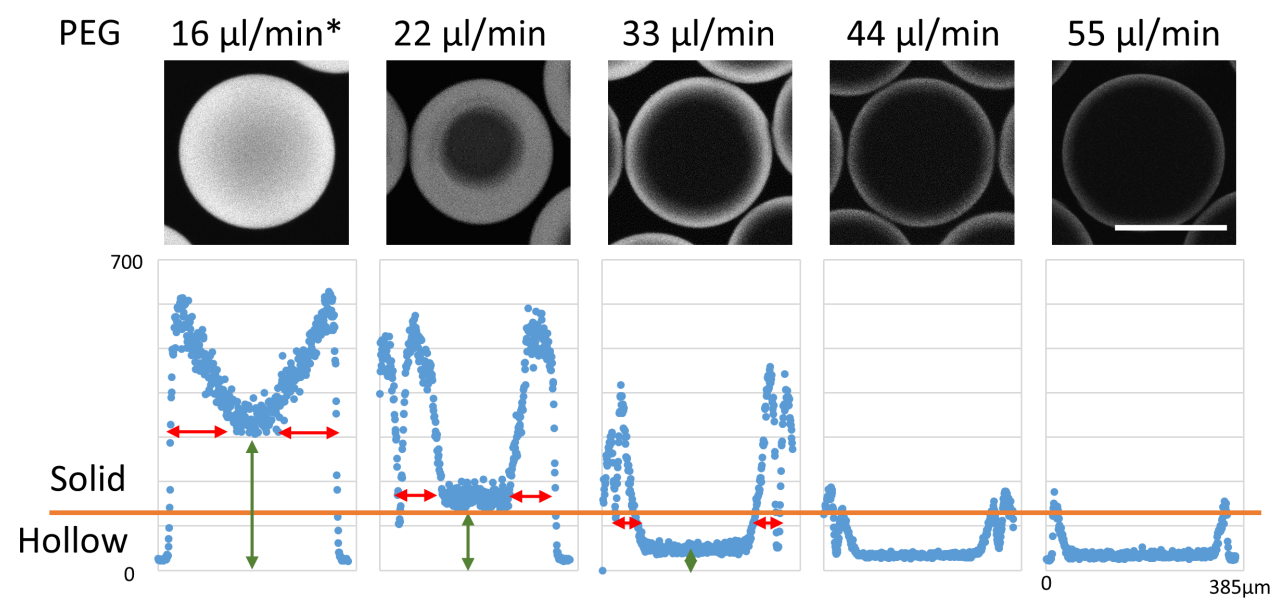

Figure 2: Upper panel: Photomicrographs of individual PEG microgels stained with ethidium homodimer obtained at various flow speeds determining the retention time in the $\mathrm{H} 2 \mathrm{O} 2$ tubing. Fluorescence intensity is a direct measure of crosslinking density. Lower panel: Quantification of the upper panel in which the fluorescence intensity of a confocal midsagittal section of the microgel is plotted along its diameter. The red horizontal arrows indicate the shell thickness, the green vertical arrow indicates the crosslinking density in the core. The orange line indicates a cutoff between a solid and a hollow core. Scale bar represents $250 \mu \mathrm{m}$.

On the lowest flow speed, and thus the longest residence time in the $\mathrm{H}_{2} \mathrm{O}_{2}$ diffusion tubing, even the core of the PEG gel is substantially crosslinked, indicated by the high fluorescent signal in the core, as indicated by the green line in the plot (Figure 2). Increasing the flow speed to $22 \mu \mathrm{l} / \mathrm{min}$ resulted in a more core-shell like gel, however, the core was still slightly crosslinked. From $33 \mu \mathrm{l} / \mathrm{min}$ 
on, the shell (Figure 2, red lines) is thin and the core is hollow. Higher flow speeds than $55 \mathrm{\mu l} / \mathrm{min}$, and thus shorter residence times, resulted in insufficient crosslinking leading to a large amount of broken capsules. Thus the residence time in the $\mathrm{H}_{2} \mathrm{O}_{2}$ diffusion tubing determines the crosslinking density in the shell, the shell thickness and the crosslinking in the core.

The same diffusion based crosslinking worked for the DexHA gels (Figure 3). However, here the residence time mainly controlled the shell thickness and crosslinking of the core. The crosslinking density of DexHA in the shell appears less influenced by the flow speed and thus retention time in the $\mathrm{H}_{2} \mathrm{O}_{2}$ diffusion tubing compared to PEG (compare Figures 2 and 3). The crosslinking penetrated less into the core on lower flow speeds, compared to PEG capsules. Additionally, the most dense part of the crosslinking is found in a thin outer shell around the gel, and is of a relatively high density at all flow speeds. From $44 \mu \mathrm{l} / \mathrm{min}$ on the gels have a hollow core. Slightly higher flow speeds compared to PEG are possible before the capsules start to break (77 $\mu \mathrm{l} / \mathrm{min}$, not shown).

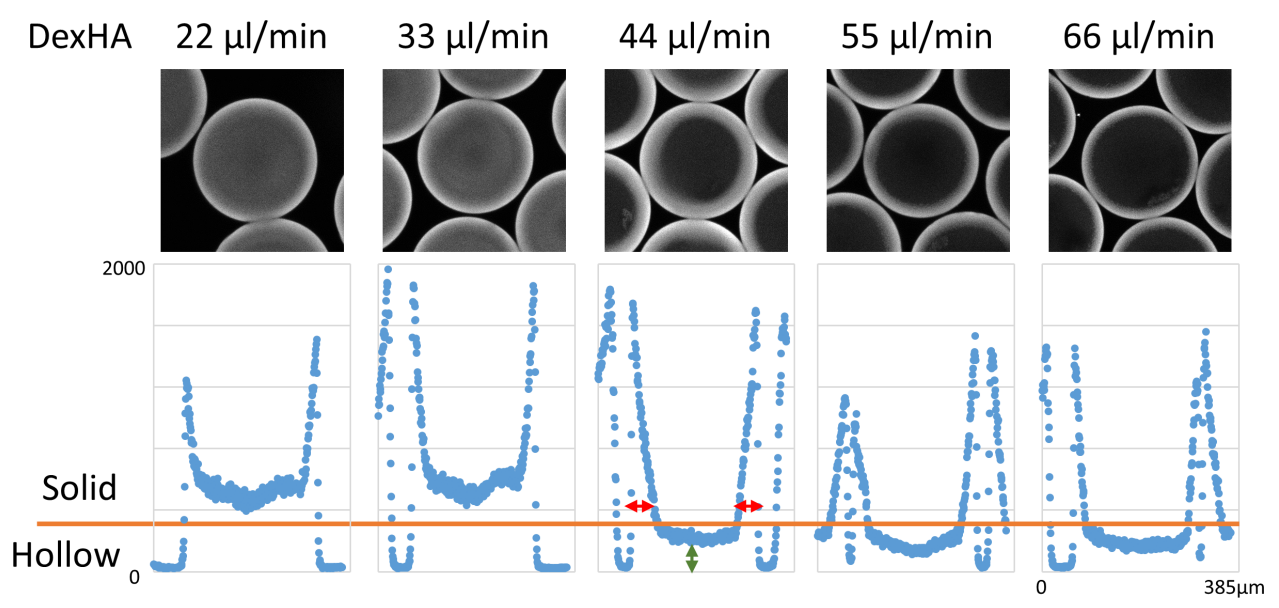

Figure 3: Upper panel: Photomicrographs of individual DexHA microgels stained with ethidium homodimer obtained at various flow speeds determining the retention time in the $\mathrm{H}_{2} \mathrm{O}_{2}$ tubing. Fluorescence intensity is a direct measure of crosslinking density. Lower panel: Quantification of the upper panel in which the fluorescence intensity of a confocal midsagittal section of the microgel is plotted along its diameter. The red horizontal arrows indicate the shell thickness, the green vertical arrow indicates the crosslinking density in the core. The orange line indicates a cutoff between a solid and a hollow core. Scale bar represents $250 \mu \mathrm{m}$. 


\section{Permeability}

Permeability of capsules is important for nutrients and waste products to diffuse in and out. Additionally, capsules can be tailored to blocking components of the immune system, making them immunoprotective ${ }^{[22]}$. In order to analyze the permeability of the gels, we used FITC conjugated dextrans in a molecular weight range of $20 \mathrm{kD}$ up to $2000 \mathrm{kD}$ and a globular IgG protein with a molecular weight of $150 \mathrm{kDa}$. We chose to analyze core-shell PEG gels and DexHA gels produced at a flow speed of $33 \mu \mathrm{l} / \mathrm{min}$ and $44 \mu \mathrm{l} / \mathrm{min}$, respectively, as these are the most robust, yet hollow gels. Analysis showed that DexHA capsules were permeable for all FITC conjugates up to $2000 \mathrm{kDa}$ and IgG (Figure 4) although diffusion is approximately $50 \%$ delayed for larger molecules. This suggests that DexHA based micro hydrogels are permeable, and most likely permeable to almost all relevant molecules in vivo, including large proteins but diffusion might be hampered. Quantification of IgG penetration in DexHA was impossible due to IgG precipitation in the core of the gel. The PEG capsules, however, were only permeable up to FITC conjugates of $150 \mathrm{kDa}$. Based on the fluorescence intensity of the core in PEG capsules incubated with dextran > 500k and with IgG which were around back ground levels, diffusion of large molecules appeared severely jeopardized in these capsules. 


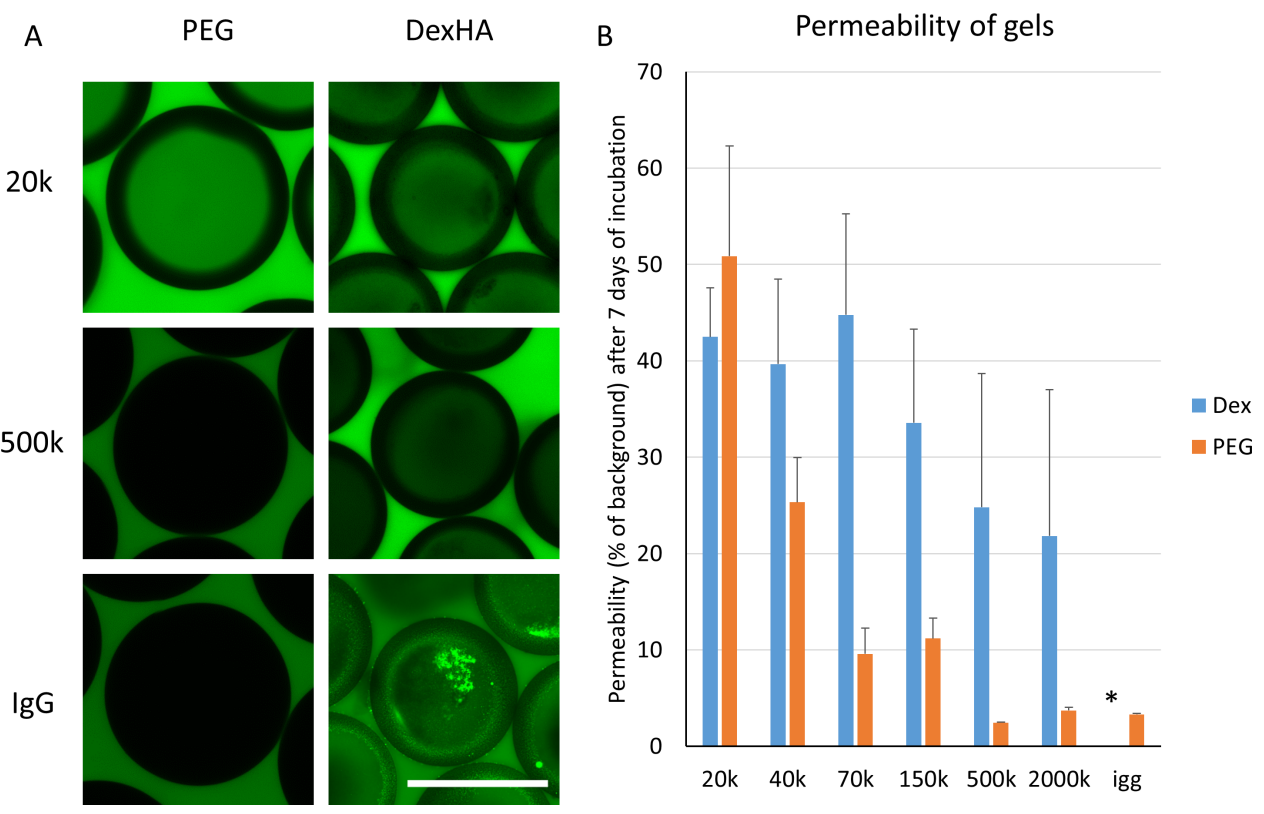

Figure 4: A) Confocal slices of hollow microgels incubated in FITC conjugated dextrans and IgG. Scale bar represents $250 \mu \mathrm{m}$. B) Quantification of the microgels' permeability by automated image analysis of the confocal images from $A(N>50)$. *DexHA gels are permeable to IgG, but quantification was impossible due to IgG precipitation in the core of the gel.

\section{Cell aggregation}

MSCs and MIN6 cells were encapsulated as single cells at high density, in DexHA gels with a slightly crosslinked core produced at a flow speed of $22 \mu \mathrm{l} / \mathrm{min}$ and in hollow gels produced at a flow speed of $44 \mu \mathrm{l} / \mathrm{min}$. We chose $22 \mu \mathrm{l} / \mathrm{min}$ as it has a slightly crosslinked homogeneous core (Figure 3), and $44 \mu \mathrm{l} / \mathrm{min}$ as the hollow core condition, as in these conditions the shell is still relatively strong, allowing for easy handling and long term culture. Cell survival was excellent even under flow conditions with relatively long retention time in the diffusion channel. This indicates that only a very small percentage of $\mathrm{H}_{2} \mathrm{O}_{2}$, in a low and non-toxic concentration, reached the cells despite the tubing was traversed through a $30 \%$ $\mathrm{H}_{2} \mathrm{O}_{2}$ solution. Once diffusing into the droplet, the $\mathrm{H}_{2} \mathrm{O}_{2}$ is rapidly consumed by the crosslinking reaction resulting in neglectable exposure of cells to $\mathrm{H}_{2} \mathrm{O}_{2}$ (Figure S1 and Kamperman et al. ${ }^{[21]}$ ). 

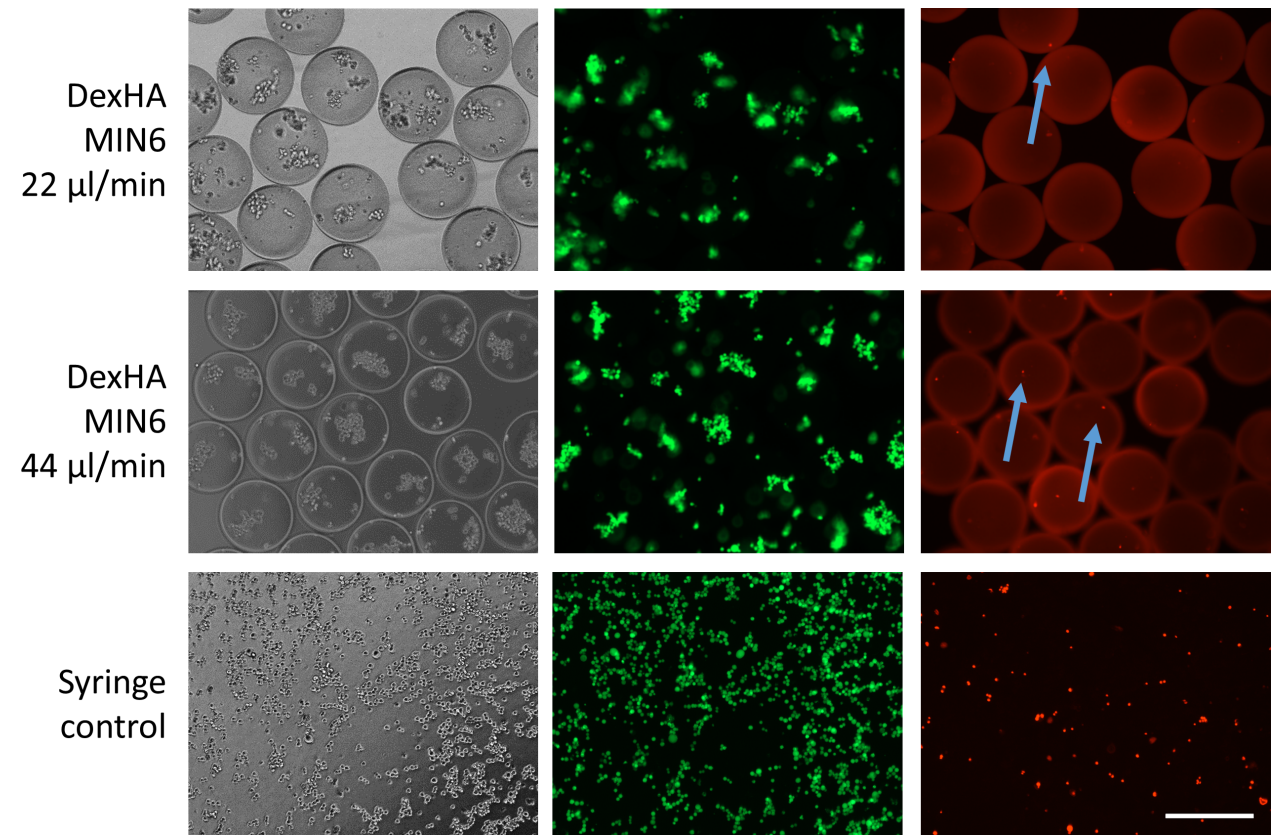

Figure S1: Epifluorescent images of live (green)/dead (red) staining of microgels at $22 \mu \mathrm{l} / \mathrm{min}, 44$ $\mu \mathrm{l} / \mathrm{min}$ ) at two different focal planes), and the syringe control. Blue arrows indicate dead cells. Scale bar represents $250 \mu \mathrm{m}$.

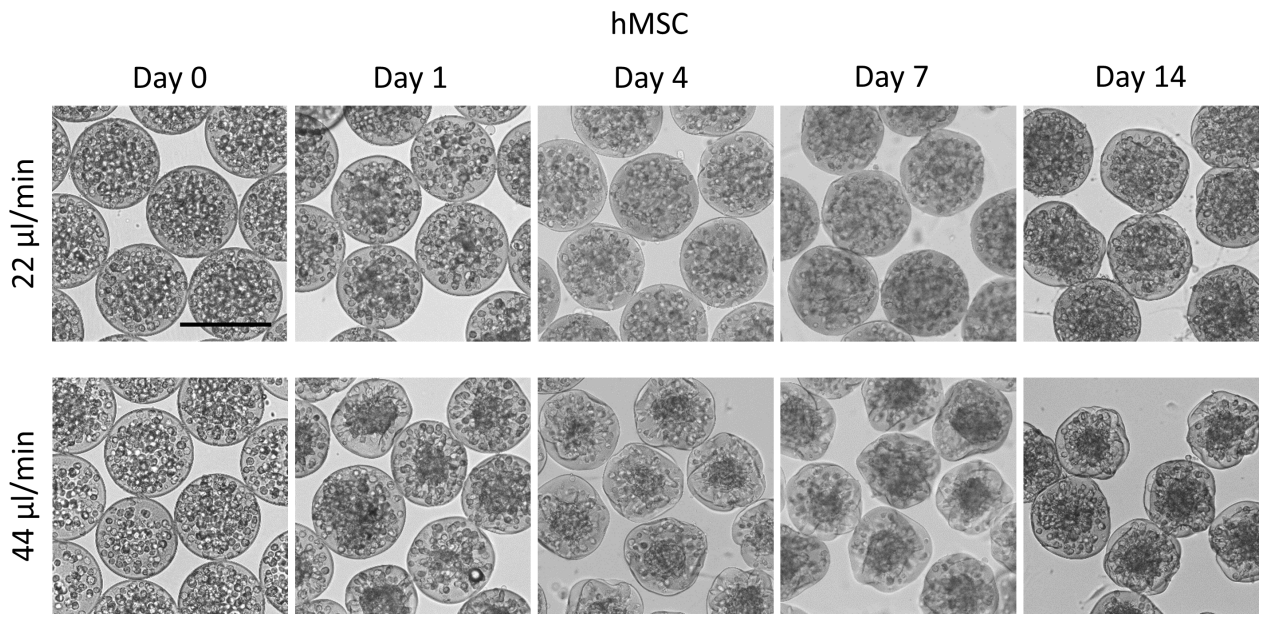

Figure 5: hMSCs at $20^{*} 10^{6}$ cells $/ \mathrm{ml}$ encapsulated in DexHA gels with a slightly crosslinked core (22 $\mu \mathrm{l} / \mathrm{min}$, top) and hollow gels $(44 \mu \mathrm{l} / \mathrm{min}$, bottom), at day $0,1,4,7$ and 14 . Scale bar represents 250 $\mu \mathrm{m}$. 
When encapsulating MSCs in the slightly crosslinked core, no aggregation is observed over 14 days of culture. At day 14 the gels are slightly deformed. In the hollow gels however, already at day 1 aggregates were formed, which deformed the outer shell of the capsule likely due to contraction of the aggregating cells.

Since MIN6 cells have a strong tendency to aggregate, it is difficult to obtain stable pure single cell solutions for encapsulation purposes. This explains why at day 0 small clumps of cells are visible in both the core-shell and the more solid gel compositions. Remarkably, while in core-shell configuration aggregation of cells continued, in the solid gel this process is clearly delayed. After one day in the hollow gels, most cells are aggregated, with few loose cells that are "stuck" into the crosslinked shell. In the slightly crosslinked core, cells started to clump together after one day, but most were still present in small groups. At day 2 , the aggregates in hollow gels were becoming spherical and started to become larger, likely due to cell proliferation. In the slightly crosslinked core gels several aggregates are forming. After a week of culture the aggregates increased in size in both conditions, and most gels contained one or two large aggregates only. There is no obvious difference anymore between the slightly crosslinked core and the hollow gels. The cell aggregates continued to increase in size and at day 14 in both conditions the whole gel was filled with a single aggregate.

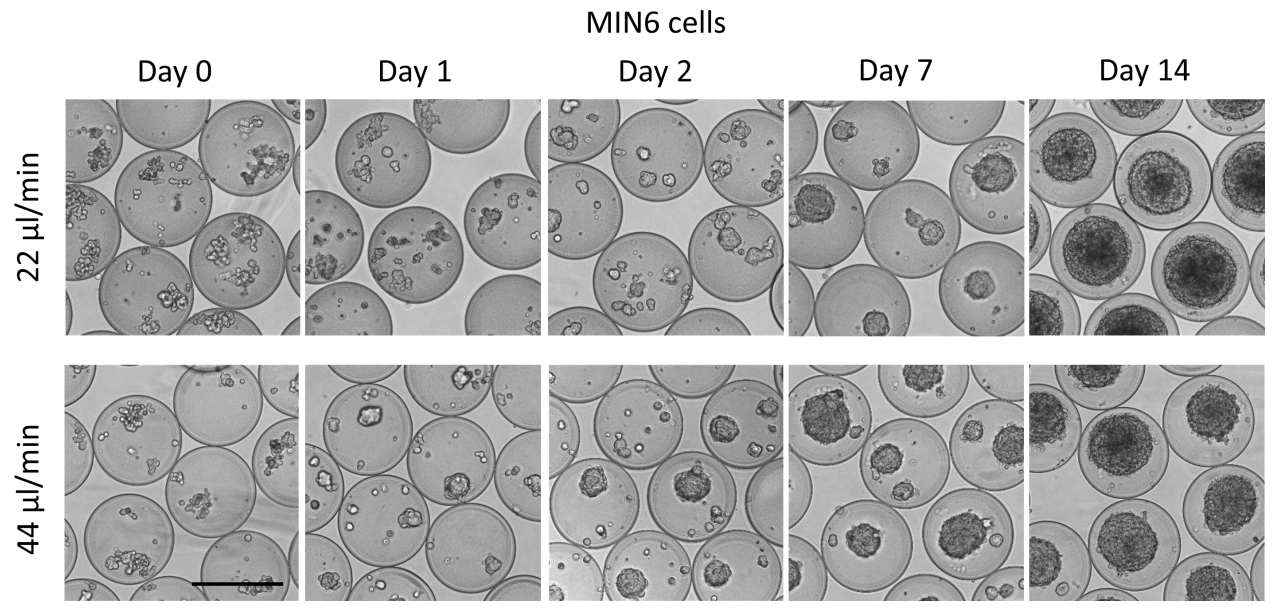

Figure 6: MIN6 cells at $12 * 10^{6}$ cells/ml encapsulated in DexHA gels with a slightly crosslinked core (22 $\mu \mathrm{l} / \mathrm{min}$, top) and hollow gels ( $44 \mu \mathrm{l} / \mathrm{min}$, bottom), at day $0,1,2,7$ and 14 . Scale bar represents $250 \mu \mathrm{m}$. 


\section{In explant cell aggregation}

When loaded with mixtures of single cells, core-shell microgel configuration could potentially be used for direct injection of capsules in vivo. The capsule would then facilitate the formation of cell aggregates in situ. To test this hypothesis an ex vivo experiment was performed using an organ culture model. After injection of the single cell suspension-laden core-shell microgels each containing approximately 250 cells in the explanted epidermal fat pad or under the kidney capsule, the organs were cultured for 24 hours to allow for cell aggregation.

Histology Day 1

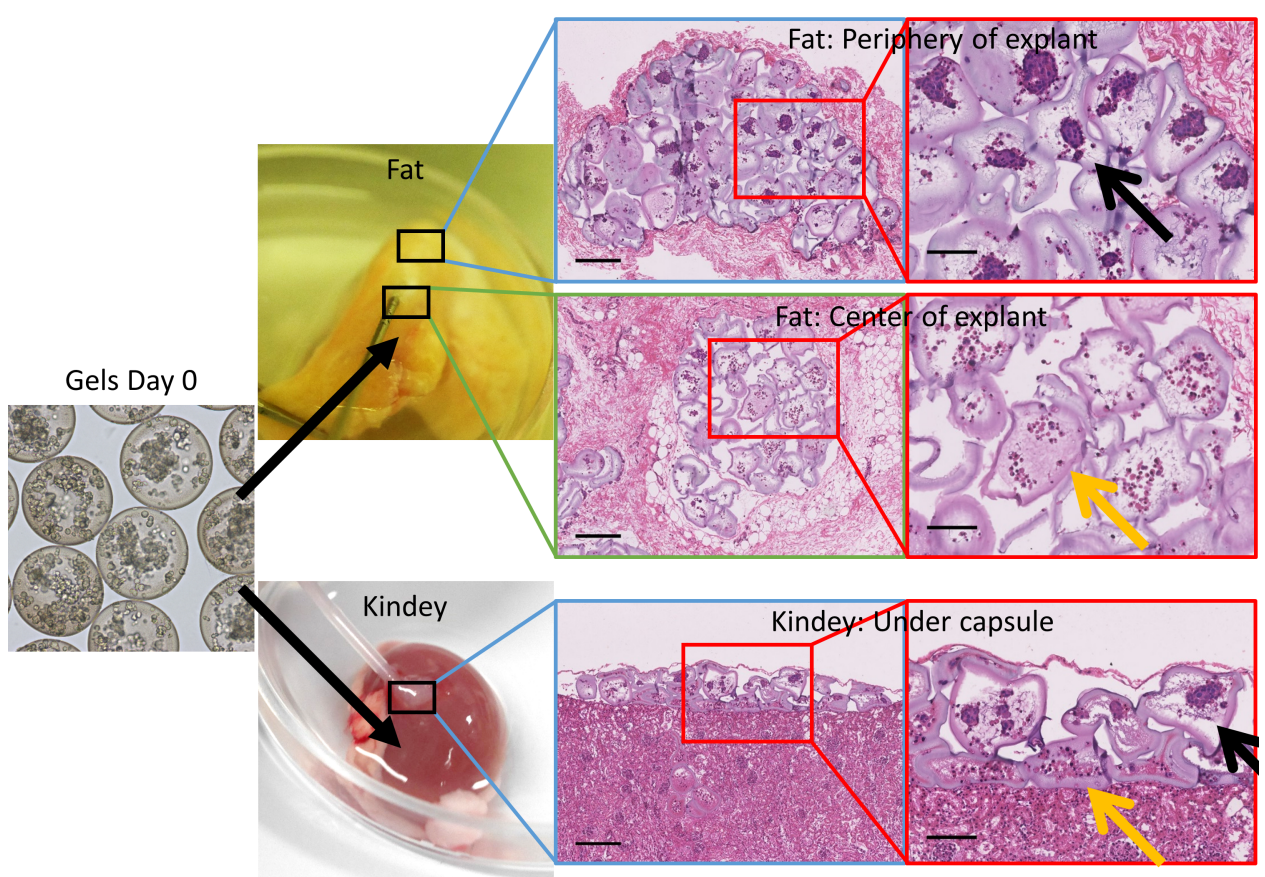

Figure 7: Left: Cell aggregation was tested by injecting hollow DexHA gels each containing approximately 250 MIN6 cells in explants of the epidermal fat pad and the kidney ex vivo. After 24hours samples were collected for histological analysis. Right: Hematoxylin \& Eosin staining of the implanted microgels in the fat pad and under the kidney capsule after 24 hours of culture. Black arrows indicate aggregate formation, yellow arrows indicate gels without aggregate formation. Scale bar represents $100 \mu \mathrm{m}$ (left) and $250 \mu \mathrm{m}$ (right). 
Histology revealed successful cell aggregation in the gels injected in the periphery of the fat pad and directly under the kidney capsule (Figure 7), indicating that this would be a feasible strategy in vivo. However, more towards the center of the fat pad and the kidney, the cells did not aggregate, most likely due to diffusion limitations, because there is no perfusion in this tissue explant model, resulting in lack of oxygen and nutrients.

\section{Discussion}

Cell aggregation is an important biological process, and is used for studying a variety of diseases, to create three-dimensional models for stem cell differentiation and drug screening, for applications in tissue engineering, and for in situ delivery of therapeutic factors. Numerous strategies are available for controlled cell aggregation, of which microwell systems are very efficient and straightforward ${ }^{[4,5]}$. However, in long term cultures aggregates need to be protected from fusing together into large clumps or attaching to the culture substrate, while allowing regular changes of culture medium. When implantating as therapeutic delivery vehicles, cells need to be confined in the right environment. Hydrogel capsules, or hollow hydrogels, can circumvent these problems and offer additionally the possibility to engineer specialized microenvironments for example by incorporating specific triggers like growth factors in the core and/or in the shell.

Here we present a robust and straightforward method to produce cellladen core-shell microgels. Our method relies on the enzymatic crosslinking of polymer-tyramine conjugates in an enzymatic reaction which is extremely fast, usually completed in a few seconds, and which is strictly dependent on the presence of the activator $\mathrm{H}_{2} \mathrm{O}_{2}$. Removal of $\mathrm{H}_{2} \mathrm{O}_{2}$ thus allows to stop the reaction on demand. This system is unique because of the outside-in diffusion of the extremely small $\mathrm{H}_{2} \mathrm{O}_{2}$, allowing to crosslink outside-in. By simply varying the flow speed of the droplets, and thus the residence time in the $\mathrm{H}_{2} \mathrm{O}_{2}$ diffusion tubing, the diffusion of the $\mathrm{H}_{2} \mathrm{O}_{2}$ into the droplets and hence the outside-in crosslinking of the polymer conjugates, can be controlled.

By further prolonging the diffusion time, $\mathrm{H}_{2} \mathrm{O}_{2}$ can penetrate deeper into the droplet resulting in crosslinking of the core of the hydrogel. Since the amount 
of $\mathrm{H}_{2} \mathrm{O}_{2}$ which diffuses through the tubing and the oil phase into the droplet is extremely limited, and once it diffuses into the droplet it is rapidly consumed by the enzyme HRP, it is ensured that cells are not exposed to toxic concentrations of $\mathrm{H}_{2} \mathrm{O}_{2}$, making this method for preparing cell laden core-shell crosslinked hydrogels very cell friendly, as long as the inside of the gel is not yet fully crosslinked. Even with a slightly crosslinked core, cell survival is excellent exceeding $>90 \% 4$ hours after production (Figure S1). Long term survival is also excellent with neglectable cell death, which is supported by normal cell responses such as spontaneous aggregation of encapsulated cells over time and growth of cell aggregates.

Compared to the two enzyme system with catalase and HRP from Ashida and coworkers ${ }^{[12]}$, our system is easier to tune and only needs one enzyme. Also our system is more flexible, as the $\mathrm{H}_{2} \mathrm{O}_{2}$ is supplied via diffusion, and not dependent on the flow rate of unstable $\mathrm{H}_{2} \mathrm{O}_{2}$ oil emulsions. Compared to other methods described for production of core-shell microgels, which require access to nano and microfabrication technology, our method relies on the use of of-theshelve components, requiring only little modification in a normal workshop, and can thus be easily adapted in any standard tissue culture laboratory. Furthermore, the method is robust and cell laden core-shell microgels can be easily produced at a speed of over 500 microgels per minute.

Our method for the production of core-shell microgels is extremely versatile. A wide variety of natural and synthetic tyramine polymer conjugates have previously been used in a HRP mediated enzymatic crosslinking reaction to produce stable macromolecular networks. Indeed besides dextran, HA and PEG as used in this study tyramine conjugates of, heparin ${ }^{[23]}$, chitosan ${ }^{[24]}$, alginate ${ }^{[25]}$, gelatin, and Poly Vinyl Alcohol ${ }^{[26]}$ have been reported in literature. These polymer conjugates could be used in our method. Hence it will be possible to tailor the biological and physicochemical properties of the shell by varying in the combinations of polymer conjugates.

This versatility opens the unique opportunity to engineer specialized microenvironments for controlled cell aggregation for example by incorporating specific triggers like growth factors in the core and/or in the shell. Additionally, the hydrogel shell can be tuned into a specialized interface between the encapsulated cells and the outside environment. The shell can protect its content 
from the immune system in e.g. allogenic cell transplantation or islet transplantation ${ }^{[22]}$, from physical threads to the cells like shear stress and/or compression. Indeed, when we used PEG for production of core-shell microgels diffusion of IgG antibodies into the gels was neglectable, suggesting that the shell could potential act as an immunoprotective shield. The shell can also be optimized for interaction with the surrounding tissue upon implantation of the core-shell microgels, for example by facilitating its integration in tissues or by stimulation of the attraction and guidance of blood vessels to the microgels ${ }^{[27]}$.

Core-shell microgels could be engineered to act as dedicated microbioreactors boosting functions of encapsulated cells, both in vitro and in vivo. We show that we can control the speed of aggregation of encapsulated cells by varying the crosslinking density of the core. This aggregation test can be used to study the aggressiveness or invasiveness of cancer cells ${ }^{[13,28-30]}{ }^{\text {. Aggregation }}$ of cells in the microbioreactors can also occur in vivo. Indeed, in a proof of principle experiment we demonstrated that cells encapsulated in the core-shell microgels successfully aggregated within 24 hours in an organ explant model. This shows that the principle of in situ cell aggregation can also work in an in vivo situation. This implies that the core of the gels can potentially be used to provide the right environment for the in vivo differentiation of encapsulated stem cells, while the shell helps to contain the cells and protect them from the immune system and instant blood mediated responses. As such the gels can be used for the in vivo delivery of "living cell factories" [31], where encapsulated cells produce and release therapeutic agents.

Boosting of cell function in core-shell microgels could also be facilitated by principles like macromolecular crowding. Here the shell acts as a barrier to retain secreted growth factors and extracellular matrix molecules by the encapsulated cells in the core of the microgel. For example, it has been shown that macromolecular crowding can improve differentiation of mesenchymal stromal cells ${ }^{[32-34]}$. Additionally various stimuli could also be co-encapsulated with the cells in the core of the microgel. Here the shell acts as a barrier to prevent the rapid loss of these stimuli preserving their biological activity. These functions could be further improved by modification of the shell with for example growth factor binding polymers like heparin which will result in sequestering of these growth factors in the microgels ${ }^{[35]}$. As we show by using PEG instead of DexHA, 
the permeability of the shell of the gels can be changed drastically. Besides its functional properties, the thickness of the shell and the crosslinking density of the core can be tuned by varying the residence time in the $\mathrm{H}_{2} \mathrm{O}_{2}$. Increased thickness of the shell could be feasible in for example implantations where higher mechanical stability is required, like bone or cartilage.

In conclusion, we present here a flexible and versatile method for the production of cell laden core-shell microgels. By the independent tuning of the properties of the core and the shell specialized microenvironments can be engineered which are optimized for interaction with the surrounding environment as well as optimized to support the function of the encapsulated cells. These cell-laden hollow microgels can act as microbioreactors with a great potential in various biomedical applications both in vitro and in vivo. 


\section{Acknowledgements}

The authors acknowledge the support of Brenda Bakker, Cindy Kelder and Betty Klomphaar for help with organ explantation.

\section{References}

[1] F. Wolf, C. Candrian, D. Wendt, J. Farhadi, M. Heberer, I. Martin, and a Barbero, "Cartilage tissue engineering using pre-aggregated human articular chondrocytes.", European cells \& materials, vol. 16, pp. 92-9, 2008.

[2] J. Friedrich, C. Seidel, R. Ebner, and L. A. Kunz-Schughart, "Spheroid-based drug screen: considerations and practical approach", Nat. Protocols, vol. 4, no. 3, pp. 309-324, 2009.

[3] H. Kurosawa, "Methods for inducing embryoid body formation: in vitro differentiation system of embryonic stem cells.", Journal of bioscience and bioengineering, vol. 103, no. 5, pp. 389-98, 2007.

[4] J. Hilderink, S. Spijker, F. Carlotti, L. Lange, M. Engelse, C. van Blitterswijk, E. de Koning, M. Karperien, and A. van Apeldoorn, "Controlled aggregation of primary human pancreatic islet cells leads to glucose-responsive pseudoislets comparable to native islets.", Journal of cellular and molecular medicine, vol. 19, no. 8, pp. 1836-46, 2015.

[5] L. S. Moreira Teixeira, J. C. H. Leijten, J. Sobral, R. Jin, a. a. van Apeldoorn, J. Feijen, C. van Blitterswijk, P. J. Dijkstra, and M. Karperien, "High throughput generated micro-aggregates of chondrocytes stimulate cartilage formation in vitro and in vivo", Eur Cell Mater, vol. 23, pp. 387399, 2012.

[6] M. D. Ungrin, C. Joshi, A. Nica, C. Bauwens, and P. W. Zandstra, "Reproducible, ultra high-throughput formation of multicellular organization from single cell suspension-derived human embryonic stem cell aggregates", PLOS ONE, vol. 3, no. 2, 2008.

[7] S. Pokutta and W. I. Weis, "Structure and Mechanism of Cadherins and Catenins in Cell-Cell Contacts", Annual Review of Cell and Developmental Biology, vol. 23, pp. 237-261, 2007.

[8] L. Y. Wu, D. Di Carlo, and L. P. Lee, "Microfluidic self-assembly of tumor spheroids for anticancer drug discovery", Biomedical Microdevices, vol. 10, no. 2, pp. 197-202, 2008.

[9] S. Henke, J. Leijten, E. Kemna, M. Neubauer, A. Fery, A. van den Berg, A. van Apeldoorn, and M. Karperien, "Enzymatic Crosslinking of Polymer Conjugates is Superior over Ionic or UV Crosslinking for the On-Chip Production of Cell-Laden Microgels", Macromolecular Bioscience, vol. 16, 
no. 10 , pp. 1524-1532, 2016.

[10] D. Velasco, E. Tumarkin, and E. Kumacheva, "Microfluidic Encapsulation of Cells in Polymer Microgels", Small, vol. 8, no. 11, pp. 1633-1642, 2012.

[11] C. Kim, S. Chung, Y. E. Kim, K. S. Lee, S. H. Lee, K. W. Oh, and J. Y. Kang, "Generation of core-shell microcapsules with three-dimensional focusing device for efficient formation of cell spheroid", Lab on a Chip, vol. 11, no. 2, pp. 246-252, 2011.

[12] T. Ashida, S. Sakai, and M. Taya, "Competing two enzymatic reactions realizing one-step preparation of cell-enclosing duplex microcapsules", Biotechnology Progress, vol. 29, no. 6, pp. 1528-1534, 2013.

[13] K. Alessandri, B. R. Sarangi, V. V. Gurchenkov, B. Sinha, T. R. Kießling, L. Fetler, F. Rico, S. Scheuring, C. Lamaze, A. Simon, S. Geraldo, D. Vignjevic, H. Doméjean, L. Rolland, A. Funfak, J. Bibette, N. Bremond, and P. Nassoy, "Cellular capsules as a tool for multicellular spheroid production and for investigating the mechanics of tumor progression in vitro.", Proceedings of the National Academy of Sciences of the United States of America, vol. 110, no. 37, pp. 14843-8, 2013.

[14] R. a. Perez and H.-W. Kim, "Core-shell designed scaffolds for drug delivery and tissue engineering", Acta Biomaterialia, vol. 21, no. 0, pp. 2-19, 2015.

[15] X. Zhang, W. Wang, W. Yu, Y. Xie, X. Zhang, Y. Zhang, and X. Ma, "Development of an in vitro multicellular tumor spheroid model using microencapsulation and its application in anticancer drug screening and testing", Biotechnology Progress, vol. 21, no. 4, pp. 1289-1296, 2005.

[16] S. Sakai, I. Hashimoto, and K. Kawakami, "Production of cell-enclosing hollow-core agarose microcapsules via jetting in water-immiscible liquid paraffin and formation of embryoid body-like spherical tissues from mouse ES cells enclosed within these microcapsules", Biotechnology and Bioengineering, vol. 99, no. 1, pp. 235-243, 2008.

[17] S. Sakai, S. Ito, Y. Ogushi, I. Hashimoto, N. Hosoda, Y. Sawae, and K. Kawakami, "Enzymatically fabricated and degradable microcapsules for production of multicellular spheroids with well-defined diameters of less

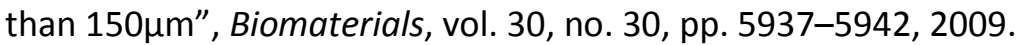

[18] S. K. Both, A. J. C. van der Muijsenberg, C. a van Blitterswijk, J. de Boer, and J. D. de Bruijn, "A rapid and efficient method for expansion of human mesenchymal stem cells", Tissue Eng, vol. 13, no. 1, pp. 3-9, 2007.

[19] J. W. H. Wennink, K. Niederer, A. I. Bochyńska, L. S. Moreira Teixeira, M. Karperien, J. Feijen, and P. J. Dijkstra, "Injectable Hydrogels by Enzymatic Co-Crosslinking of Dextran and Hyaluronic Acid Tyramine Conjugates", Macromolecular Symposia, vol. 309-310, no. 1, pp. 213-221, 2011.

[20] R. Wang, X. Huang, P. J. Dijkstra, and M. Karperien, "Enzymatic cocrosslinking of star-shaped poly(ethylene glycol) tyramine and hyaluronic 
acid tyramine conjugates provides elastic biocompatible and biodegradable hydrogels", In Preparation, 2015.

[21] T. Kamperman, S. Henke, M. Neubauer, A. Fery, J. Leijten, and M. Karperien, "Engineering 3D Single Stem Cell Microniches With Dynamically Tunable Stiffness", In Preparation.

[22] P. de Vos, M. M. Faas, B. Strand, and R. Calafiore, "Alginate-based microcapsules for immunoisolation of pancreatic islets.", Biomaterials, vol. 27, no. 32, pp. 5603-17, 2006.

[23] R. Jin, L. S. Moreira Teixeira, P. J. Dijkstra, C. a. van Blitterswijk, M. Karperien, and J. Feijen, "Chondrogenesis in injectable enzymatically crosslinked heparin/dextran hydrogels", Journal of Controlled Release, vol. 152, no. 1, pp. 186-195, 2011.

[24] R. Jin, L. S. Moreira Teixeira, P. J. Dijkstra, M. Karperien, C. A. van Blitterswijk, Z. Y. Zhong, and J. Feijen, "Injectable chitosan-based hydrogels for cartilage tissue engineering", Biomaterials, vol. 30, no. 13, pp. 2544-2551, 2009.

[25] S. Sakai and K. Kawakami, "Synthesis and characterization of both ionically and enzymatically cross-linkable alginate", Acta Biomaterialia, vol. 3, no. 4, pp. 495-501, 2007.

[26] K. S. Lim, M. H. Alves, L. a. Poole-Warren, and P. J. Martens, "Covalent incorporation of non-chemically modified gelatin into degradable PVAtyramine hydrogels", Biomaterials, vol. 34, no. 29, pp. 7097-7105, 2013.

[27] M. Najjar, V. Manzoli, M. Abreu, C. Villa, M. M. Martino, R. D. Molano, Y. Torrente, A. Pileggi, L. Inverardi, C. Ricordi, J. A. Hubbell, and A. A. Tomei, "Fibrin gels engineered with pro-angiogenic growth factors promote engraftment of pancreatic islets in extrahepatic sites in mice", Biotechnology and Bioengineering, vol. 112, no. 9, pp. 1916-1926, 2015.

[28] E. Kaemmerer, F. P. W. Melchels, B. M. Holzapfel, T. Meckel, D. W. Hutmacher, and D. Loessner, "Gelatine methacrylamide-based hydrogels: An alternative three-dimensional cancer cell culture system", Acta Biomaterialia, vol. 10, no. 6, pp. 2551-2562, 2014.

[29] F. Xu and K. J. L. Burg, "Three-dimensional polymeric systems for cancer cell studies", Cytotechnology, vol. 54, no. 3, pp. 135-143, 2007.

[30] A. Nyga, U. Cheema, and M. Loizidou, "3D tumour models: Novel in vitro approaches to cancer studies", Journal of Cell Communication and Signaling, vol. 5, no. 3, pp. 239-248, 2011.

[31] S. M. Naqvi, S. Vedicherla, J. Gansau, T. Mcintyre, M. Doherty, and C. T. Buckley, "Living Cell Factories- Electrosprayed Microcapsules and Microcarriers for Minimally Invasive Delivery", Advanced Materials, 2015.

[32] C. Chen, F. Loe, A. Blocki, Y. Peng, and M. Raghunath, "Applying macromolecular crowding to enhance extracellular matrix deposition and 
its remodeling in vitro for tissue engineering and cell-based therapies", Advanced Drug Delivery Reviews, vol. 63, no. 4, pp. 277-290, 2011.

[33] M. C. Prewitz, A. Stißel, J. Friedrichs, N. Träber, S. Vogler, M. Bornhäuser, and C. Werner, "Extracellular matrix deposition of bone marrow stroma enhanced by macromolecular crowding", Biomaterials, vol. 73, pp. 60-69, 2015.

[34] A. S. Zeiger, F. C. Loe, R. Li, M. Raghunath, and K. J. van Vliet, "Macromolecular crowding directs extracellular matrix organization and mesenchymal stem cell behavior", PLOS ONE, vol. 7, no. 5, 2012.

[35] D. B. Pike, S. Cai, K. R. Pomraning, M. A. Firpo, R. J. Fisher, X. Z. Shu, G. D. Prestwich, and R. A. Peattie, "Heparin-regulated release of growth factors in vitro and angiogenic response in vivo to implanted hyaluronan hydrogels containing VEGF and bFGF", Biomaterials, vol. 27, no. 30, pp. 5242-5251, 2006. 


\section{Chapter 6}

\section{Centering single cells in spherical microgels by}

uncoupling on-chip emulsification and enzymatic crosslinking using hydrogen peroxide diffusion

Tom Kamperman*, Sieger Henke*, Niels Ruiterkamp, Pieter J Dijkstra, Marcel Karperien**, Jeroen Leijten**

* TK and SH are shared first author

** JL and MK are shared senior author 


\section{Abstract}

Single-cell-laden microgels support physiological 3D culture conditions while enabling straightforward handling and high-resolution read-outs of individual cells. However, several technical challenges such as cell escape due to off-center encapsulation, inhomogeneous curing of microgels, and droplet generator clogging have hampered the widespread adoption of single cell encapsulation methods. These challenges are the direct result of coupled emulsification and gelation; a strategy that is used in most state-off-the-art microgel production platforms. Here, we present a novel modular microfluidic platform to uncouple emulsification and gelation of enzymatically crosslinkable microgels, thereby enabling homogeneous crosslinking while preventing clogging. Moreover, delayed crosslinking drives cellular repositioning from the droplets' oil/water interface to the droplet's center, which provides ultimate control over biochemical and biomechanical stimulation of single cells in a physiological 3D microenvironment. Our novel strategy supports homogenous encapsulation, autonomous centering, and long-term ( 28 days) in vitro culture of individual mesenchymal stem cells in dextran-tyramine microgels, without adversely affecting cell viability. The ability to center single cells in microgels using our modular microfluidic chip approach will aid in driving rapid widespread adoption of single cell microencapsulation technology. 


\section{Introduction}

Encapsulating cells into biomaterials such as hydrogels provides cells with an extracellular matrix that can be tuned to mimic natural microenvironments in vitro ${ }^{[1-3]}$. Engineering the physicochemical and biofunctional properties of a biomaterial provides control over cellular behaviors including migration, survival, proliferation, and differentiation ${ }^{[4-6]}$. Consequently, cell-laden hydrogels have a great potential to contribute to fundamental biological studies, pharmacological screenings, and cell-based therapies ${ }^{[7-9]}$. Although the size of cell encapsulating biomaterials is typically in the millimeter to centimeter range, it has been recognized that downsizing the construct to the micrometer scale, called microgels, has the potential to advance many applications. In particular, the production of microgels that contain a single cell is expected to open up a myriad of novel avenues. Single-cell-laden microgels can, for example, advance the highthroughput screening of cell-material combinations by lowering material costs, improving diffusion rates, and allowing for facile single cell analysis ${ }^{[10]}$. Furthermore, cell-laden microgels could function as modular building blocks for engineering tissues with intrinsic multiscale hierarchy, which is essential for the functioning of native tissues ${ }^{[11]}$. Ultimately, encapsulating individual cells in microgels that are only a few micrometers larger than the cells they encapsulate offers unique advantages. Microgels encapsulating an individual cell are readily compatible with standard visualization techniques such as confocal microscopy, without the need for physical or optical processing such as sectioning due to their minimal height. They also offer highly efficient material-to-cell volume ratios, improved diffusion rates of solutes, and can potentially enable real-time pharmacological screenings while maintaining 3D culture conditions.

Droplet microfluidics technology is ideally suited for the production of cell-laden microgels with narrow size distributions ${ }^{[12]}$. However, the potential of droplet microfluidics to generate single-cell-laden microgels is currently limited. Importantly, all state-of-the-art single cell microencapsulation platforms are characterized by suboptimal cell encapsulation. During droplet formation, cells take position at the droplet's water/oil interface immediately after emulsification, resulting in partial cell encapsulation and even the escape of cells upon gelation ${ }^{[13-18]}$. Positioning cells off-center in microgels will expose the cells non-symmetrically to biochemical and biomechanical stimulation. In addition, 
incomplete encapsulation will expose the entrapped cell to the external environment, which counteracts possible functions of the microgels such as immunoprotection ${ }^{[19]}$. Encapsulation of single-cell microgels in a second layer of biomaterial has been explored to prevent cell escape and guarantee immunoprotection ${ }^{[17,20]}$. However, this two-layer approach increases the microgels' size and still exposes the cells to a polarized microenvironment.

Cell encapsulation in microgels using microfluidic droplet generation typically relies on chemical-based in situ gelating hydrogels. Often, gel precursor and crosslinker solutions are mixed on-chip to initiate gelation and subsequently emulsified using a microfluidic droplet generator. As such, emulsification and gelation are coupled and occur near-simultaneously, which can cause clogging of the droplet generator and result in inhomogeneous crosslinking of microgels ${ }^{[21]}$. Uncoupling (i.e. physically separating) the gelation and emulsification addresses these problems, and can be achieved via delayed crosslinking of the microgels. Feasibility of the delayed crosslinking approach has been previously reported. Specifically, delayed acid induced crosslinking of alginate was used to create relatively large $(100 \mu \mathrm{m})$ multi cellular microgels to mitigate challenges faced with conventional $\mathrm{CaCl}_{2}$ crosslinking. This solution has been successfully applied for the production of alginate microgels, where in situ gelation post emulsification was achieved using the triggered dissolution of calcium carbonate nanoparticles by supplementing the gel precursor droplets with acidic oil ${ }^{[22]}$. Unfortunately, such a delayed triggered gelation strategy has not been reported for enzymatically crosslinkable microgels, which have several advantages over widely used physical and chemical crosslinking systems.

Here, we present a novel modular microfluidic diffusion platform for the enzyme-based production of dextran-tyramine (Dex-TA) microgels containing a single centered cell using a process where emulsification and gelation are uncoupled. In contrast to ionic crosslinking (e.g. alginate), enzyme-based crosslinking forms stable covalently crosslinked polymer networks. We have previously demonstrated that enzymatically crosslinked hydrogels and microgels support cell function and tissue formation in both in vitro and in vivo applications, and even outperform the widely used physical and photocrosslinking systems used for hydrogels such as alginate and PEGDA in terms of cell survival and metabolic activity ${ }^{[23-26]}$. Consequently, the development of a novel microgel 
production platform with uncoupled emulsification and enzyme-based crosslinking would facilitate single cell encapsulation and boost widespread adoption of this cytocompatible encapsulation mechanism.

\section{Materials and methods}

\section{Materials}

Dextran-tyramine (Dex-TA, 15-25 kDa, DS15) was synthesized as previously described ${ }^{[27]}$. Horseradish peroxidase (HRP, type $\mathrm{VI}$ ), $\mathrm{H}_{2} \mathrm{O}_{2}$ (with inhibitor), ascorbic acid, iodixanol (OptiPrep), Calcein-AM, ethidium homodimer1 (EthD-1), dextran-FITC (2000 kDa), formalin, Triton X-100, and 10-Acetyl-3,7dihydroxyphenoxazine (Amplex Red) were purchased from Sigma-Aldrich. Phosphate-buffered saline (PBS) and fetal bovine serum (FBS) were purchased from Lonza. Dulbecco's Modified Eagle's Medium (DMEM), Minimal Essential Medium $\alpha$ with nucleosides ( $\alpha$ MEM), Penicillin with Streptomycin, GlutaMAX, 2mercaptoethanol, and trypsin-EDTA were purchased from Gibco. Basic fibroblast growth factor (ISOKine bFGF) was purchased from Neuromics. Phalloidin-AF488 was purchased from Molecular Probes. DRAQ5 was purchased from Thermo Scientific. Polydimethylsiloxane (PDMS, Sylgard 184) was purchased from Dow Corning. Aquapel was purchased from Vulcavite. Pico-Surf 1 in Novec 7500 Engineered Fluid and Pico-Break 1 were purchased from Dolomite. Surfactantfree fluorocarbon oil (Novec 7500 Engineered Fluid) was kindly provided by the BIOS Lab-on-a-Chip group.

\section{Cell isolation and expansion}

Human mesenchymal stem cells (MSCs) were isolated from fresh bone marrow samples and cultured as previously described ${ }^{283}$. The use of patient material was approved by the local ethical committee of the Medisch Spectrum Twente and informed written consent was obtained for all samples. In short, nucleated cells in the bone marrow aspirates were counted, seeded in tissue culture flasks at a density of 500.000 cells $/ \mathrm{cm}^{2}$ and cultured in MSC proliferation medium, consisting of $10 \% \mathrm{FBS}, 100 \mathrm{U} / \mathrm{ml}$ Penicillin with $100 \mathrm{mg} / \mathrm{ml}$ Streptomycin, 1 \% GlutaMAX, 0,2 mM ascorbic acid and $1 \mathrm{ng} / \mathrm{ml}$ bFGF (added fresh) in aMEM. When MSC culture reached near confluence, the cells were 
detached using $0,25 \%$ Trypsin-EDTA at $37{ }^{\circ} \mathrm{C}$ and subsequently subcultured or used for experimentation.

\section{Microgel production}

All microfluidic chips were manufactured from PDMS and glass using standard soft lithography techniques. The conventional microgel production, droplet generator, and $\mathrm{H}_{2} \mathrm{O}_{2}$ diffusion chips were fabricated with $\sim 25 \mu \mathrm{m}, \sim 25 \mu \mathrm{m}$, and $\sim 100 \mu \mathrm{m}$ high channels, respectively. Aquapel was introduced in the chips before usage to ensure channel wall hydrophobicity. Chips were connected to gastight syringes (Hamilton) using FEP tubing (inner diameter $250 \mu \mathrm{m}$, DuPont), which were controlled by low pressure syringe pumps (neMESYS, Cetoni). All emulsions were produced using 2 \% Pico-Surf 1 in Novec 7500 Engineered Fluid. The conventional microgel production platform was operated as described previously, using flow rates of $0,5,0,11,0,11$, and $2,8 \mu \mathrm{l} / \mathrm{min}$ for Dex-TA, HRP, $\mathrm{H}_{2} \mathrm{O}_{2}$, and oil, respectively ${ }^{[24]}$.

To uncouple emulsification and gelation, a standard droplet generator was connected to the $\mathrm{H}_{2} \mathrm{O}_{2}$ diffusion chip. In this modular microfluidic set-up, Dex-TA and HRP containing hydrogel precursor emulsion was flown through the diffusion platform, which was fed with $\mathrm{H}_{2} \mathrm{O}_{2}$ at a rate of $30 \mu \mathrm{l} / \mathrm{min}$. The $\mathrm{H}_{2} \mathrm{O}_{2}$ diffused from the feed channel through the PDMS walls into the gel precursor emulsion, thereby triggering its crosslinking. Hydrogel precursor solution was prepared by mixing $10 \%$ Dex-TA, $44 \mathrm{U} / \mathrm{ml} \mathrm{HRP}$, and $8 \%$ OptiPrep (i.e. to obtain $\rho$ $=1.05 \mathrm{~g} / \mathrm{l})$ in PBS and emulsified with surfactant containing oil at a 1:6 flow ratio. To produce cell-laden microgels, detached cells were washed with medium, flown through a $40 \mu \mathrm{m}$ cell strainer, and suspended in the hydrogel precursor solution at a concentration of $10^{7}$ cells per $\mathrm{ml}$. The cell-laden hydrogel precursor solution was loaded into an ice-cooled gastight syringe where it was continually agitated. The emulsion was broken by washing three times with surfactant free fluorocarbon oil and subsequent supplementation of Pico-Break 1 in the presence of PBS or serum containing proliferation medium. 


\section{Staining and visualization}

On-chip droplets were visualized using a stereomicroscope set-up (Nikon SMZ800 equipped with Leica DFC300 FX camera). The position of cells in microdroplets or microgels was analyzed using ImageJ software. Collected emulsions were imaged using phase contrast microscopy. To assess crosslinking homogeneity, microgels were incubated with FITC-labeled dextran after which the fluorescent intensity across microgels was measured using confocal imaging (Zeiss LSM 510) and quantified using ImageJ software. Viability of encapsulated cells was analyzed by staining with $2 \mu \mathrm{M}$ calcein-AM and $4 \mu \mathrm{M}$ EthD-1 in PBS and visualization using fluorescence microscopy (EVOS). For additional analyses, cellladen microgels were first washed with PBS and fixated using $10 \%$ formalin. Cells were permeabilized using 0,1\% Triton X-100 and subsequently stained with 2,5 $\mathrm{U} / \mathrm{ml}$ phalloidin-AF488, $50 \mu \mathrm{M}$ DRAQ5, and $4 \mu \mathrm{M}$ EthD-1 to stain F-actin, nuclei, and Dex-TA, respectively, and subsequently imaged using fluorescence confocal microscopy (Nikon A1+) and analyzed using ImageJ software.

\section{$\mathrm{H}_{2} \mathrm{O}_{2}$ detection}

To quantify $\mathrm{H}_{2} \mathrm{O}_{2}$, emulsions were broken as described before, diluted $10^{5}$ times with PBS, and mixed 1:1 with $100 \mu \mathrm{M}$ Amplex Red, 0,2 U/ml HRP in PBS. After 30 minutes incubation at room temperature, fluorescence intensity was measured using a plate reader (Victor X3, ex. 545/10 nm, em. 590/10 nm) and correlated to $\mathrm{H}_{2} \mathrm{O}_{2}$ concentrations using a standard curve.

\section{Statistical analysis}

$\mathrm{H}_{2} \mathrm{O}_{2}$ detection data is shown as average \pm standard deviation of technical triplicates. Cell positions in microdroplets and microgels were analyzed for statistical significance using ANOVA with Tukey's post hoc test with $n \geq 35$. Live/dead assay was performed on $\geq 150$ cells per time point. 


\section{Results and discussion}

\section{Challenges of single-cell-laden microgel production}

Single cell encapsulation in microgels has remained technically challenging. In situ gelation pre emulsification typically causes chip clogging, inhomogeneous crosslinking, off-center cell encapsulation, and even cell escape. To confirm and investigate these issues, we first set out to encapsulate single cells into enzymatically crosslinkable microgels using a conventional encapsulation approach. To this end, we down-sized the multi-cell enzyme-based encapsulation platform that we reported in chapter 2 of this thesis, to enable the encapsulation of individual cells in $\sim 30 \mu \mathrm{m}$ microgels ${ }^{[24]}$. Dex-TA hydrogel precursor was on-chip crosslinked via the formation of tyramine-tyramine bonds using HRP as enzyme catalyst and $\mathrm{H}_{2} \mathrm{O}_{2}$ as oxidizer (Figure $1 \mathrm{~A}$ ).

To demonstrate the potential issues of clogging and inhomogeneous crosslinking, we formed microgels using the conventional microgel production platform and a cell-free gel precursor solution. Clogging of the droplet generator was indeed frequently observed (Figure 1B). Regardless, we identified a small window of operation that enabled stable microgel production. Specifically, the concentrations and flow rates of $\mathrm{HRP}$ and $\mathrm{H}_{2} \mathrm{O}_{2}$ solutions had to be precisely tuned to prevent any gelation in front of the droplet generator. However, small disturbances of the flow immediately caused clogging and permanent chip failure, emphasizing the laborious nature of conventional microgel production platforms. Confocal microscopy of fluorescently labeled dextran microgels revealed relatively densely crosslinked regions (white arrows) that were positioned at an angle that corresponds with the HRP and $\mathrm{H}_{2} \mathrm{O}_{2}$ inlets, which indicated that the microgels were not homogeneously crosslinked (Figure 1C, D). It is likely that enzyme-mediated crosslinking was completed before thorough mixing of the droplets' components had occurred, thereby causing heterogeneities within the microgels. Such random variations in hydrogel crosslinking density can impair proper engineering of precisely controlled cellular microenvironments. 

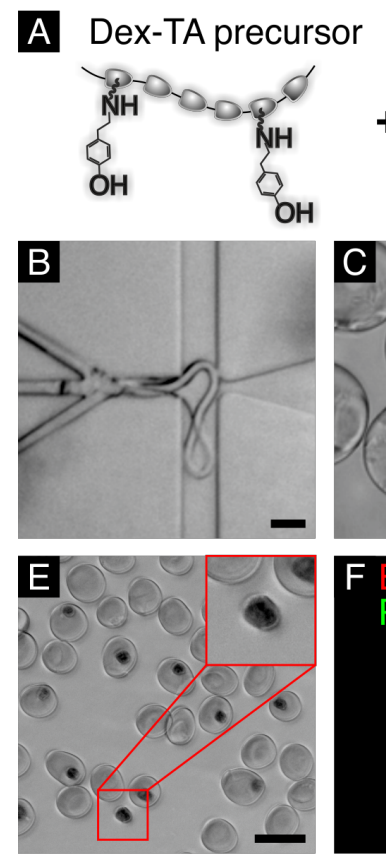
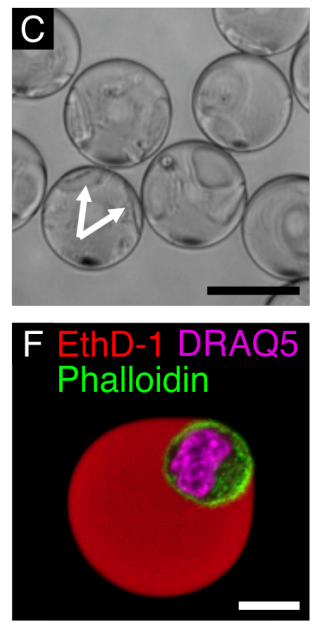

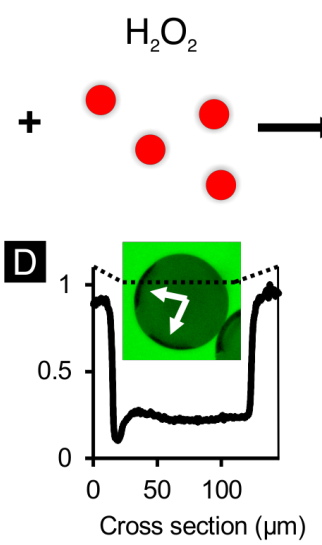

G Biochemical

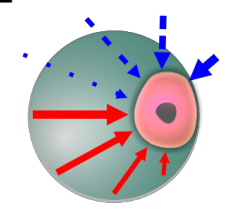

Biomechanical
Dex-TA gel
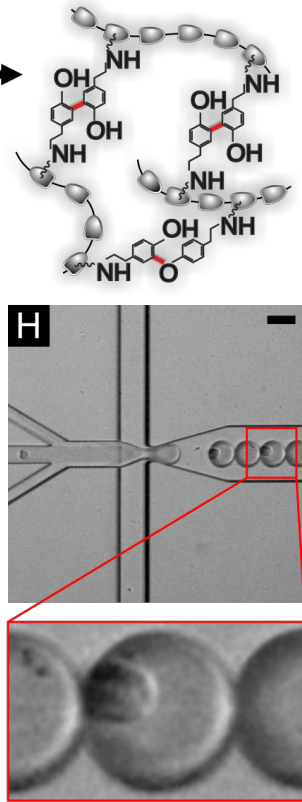

Figure 1. Typical challenges of microfluidic production of small microgels using coupled emulsification and gelation platforms. (A) Tyramine moieties of Dex-TA conjugates are crosslinked by HRP in the presence of $\mathrm{H}_{2} \mathrm{O}_{2}$. Mixing Dex-TA, HRP, and $\mathrm{H}_{2} \mathrm{O}_{2}$ pre emulsification caused (B) permanent chip clogging and $(C, D)$ inhomogeneous crosslinking of microgels, indicated by white arrows. (E) Single cell encapsulation in Dex-TA microgels frequently results in cell escape. In addition, the majority of cell-laden Dex-TA microgels contained off-center positioned cells, which was confirmed by (F) optical cross sectioning using fluorescence confocal microscopy. (G) Schematic depiction of polarized biochemical and biomechanical stimulation in off-centered single-cell-laden microgels. $(\mathrm{H})$ Cells positioned at the droplets' water/oil interface immediately after emulsification. Black scale bars depict $50 \mu \mathrm{m}$, white scale bar depicts $10 \mu \mathrm{m}$.

Using the optimized conventional production platform, we succeeded to encapsulate individual MSCs in Dex-TA microgels. As expected, the majority of cells was positioned on the outer edge of the microgels (i.e. off-center). Numerous cells were only partially encapsulated or even completely escaped the microgels during our microencapsulation attempt (Figure 1E). Suboptimal offcenter encapsulation was confirmed by optically sectioning microgels using fluorescence confocal microscopy (Figure 1F). These data complied with comparative literature by demonstrating that most single-cell-laden microgel production strategies encounter encapsulation deficiencies. Such limited control over encapsulation can hamper potential applications including fundamental cell 
studies, pharmacological screenings, and cell-based therapies. For example, offcenter encapsulated cells encounter polarized biochemical and biomechanical stimuli (e.g. drugs or biomaterials), which may evoke unnatural cell responses as compared to uniformly encapsulated cells. (Figure 1G). Furthermore, partial encapsulation and cell escape obscures conventional enzyme-based encapsulation methods from providing cells with protective environments such as immunoprotective microbarriers. Close observation of the microfluidic encapsulation procedure revealed that cells take position at the droplets' water/oil interface immediately after emulsification (Figure $1 \mathrm{H}$ ). Instantaneous gelation of these microdroplets, as used in state-of-the-art cell encapsulation procedures, locks the cells in this off-center position. Uncoupling emulsification and gelation could therefore prevent clogging and inhomogeneous crosslinking, as well as allow cells to autonomously center within microdroplets prior to crosslinking.

\section{Uncoupling emulsification and gelation}

We have developed a novel diffusion-based enzymatic crosslinking strategy to physically uncouple droplet generation from gelation. Inspired by sensor technology, we leveraged the permselective nature of silicone rubber towards $\mathrm{H}_{2} \mathrm{O}_{2}$ to control in situ enzymatic crosslinking of hydrogel precursor droplets. Specifically, we designed a dedicated microfluidic chip that consists of three equally long $(35 \mathrm{~cm})$ and high $(100 \mu \mathrm{m})$ parallel channels: one center channel for Dex-TA and HRP containing gel precursor droplets, and two aligning channels containing $\mathrm{H}_{2} \mathrm{O}_{2}$ feed solution (Figure 2A). The chip was fabricated using PDMS, indeed a silicone rubber enabling the diffusion of $\mathrm{H}_{2} \mathrm{O}_{2}$ from the feed channels towards the gel precursor droplets and initiation of enzymatic crosslinking (Figure 2B). Various combinations of different wall thicknesses and channel widths were assessed to identify the optimal design for robust in situ crosslinking of Dex-TA and HRP containing microdroplets (Figure $2 \mathrm{C}$ ).

Based on these designs, microfluidic chips were fabricated using standard soft lithography techniques and connected to a conventional microfluidic droplet generator in a modular fashion. Every design was tested for its ability to crosslink Dex-TA and HRP containing microdroplets. Chips with $25 \mu \mathrm{m}$ PDMS walls separating a $50 \mu \mathrm{m}$ wide center channel could be produced, but this design proved fragile as the thin walls often collapsed, which constrained the chip from 
further use (Figure 2D). In contrast, thicker channel walls of $50 \mu \mathrm{m}$ did not collapse during manufacture and its design readily supported the crosslinking of Dex-TA microdroplets. However, occasional droplet merging caused the formation of gel plugs, resulting in temporal stagnation of the flow (Figure 2E). Such stagnation caused differences in $\mathrm{H}_{2} \mathrm{O}_{2}$ supplementation, which is time and concentration dependent. This resulted in variation in crosslinking densities among microgels, hence the polydisperse size distribution $\left(D / D_{0}\right)$ of differently swollen microgels (Figure 2F). Chips with a $100 \mu \mathrm{m}$ center channel allowed for robust and undisturbed flow of microgel precursor droplets at all times (Figure $2 \mathrm{G})$. Finally, collected droplets and cured microgels showed equal dispersity and identical swelling rates, which indicated that all microgels had received equal amounts of crosslinker (i.e. $\mathrm{H}_{2} \mathrm{O}_{2}$ ) (Figure $2 \mathrm{H}$ ). Furthermore, the microgels were homogeneously crosslinked, as confirmed using fluorescence confocal microscopy (Figure 2I). As hypothesized, uncoupling emulsification and gelation remedied the challenge of clogging and inhomogeneous crosslinking, which are encountered with conventional enzyme-based microgel production strategies. 

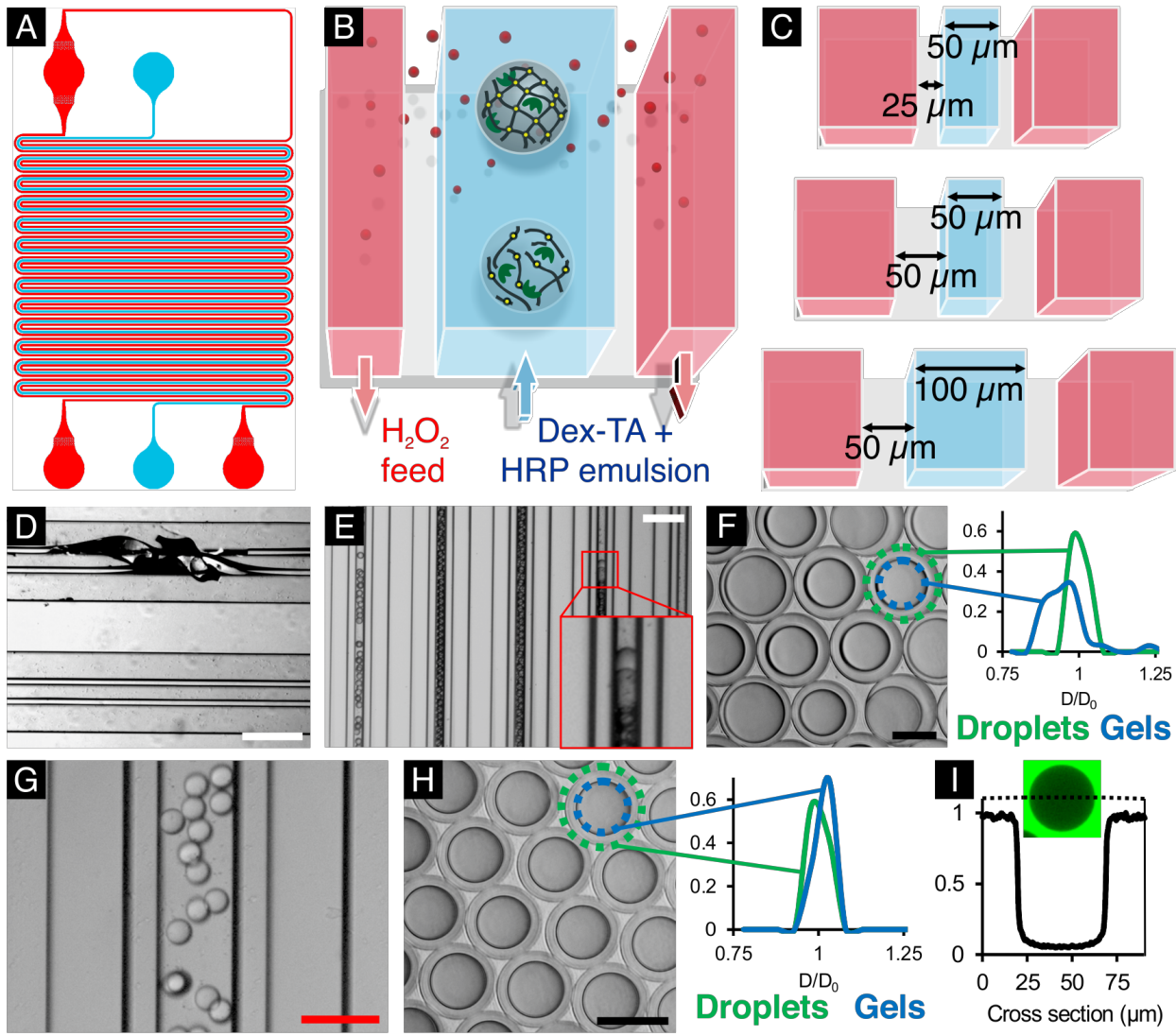

Figure 2. Design and optimization of the microfluidic $\mathrm{H}_{2} \mathrm{O}_{2}$ diffusion platform. (A) In concept, the diffusion chip consists of parallel microfluidic channels separated by a thin PDMS wall (B) enabling the controlled supplementation of $\mathrm{H}_{2} \mathrm{O}_{2}$ to initiate crosslinking of Dex-TA gel precursor droplets. (C) Various chip designs were tested to determine optimal conditions. (D) Thin $(25 \mu \mathrm{m})$ PDMS walls collapsed during chip fabrication. (E) A narrow $(50 \mu \mathrm{m})$ center channel occasionally caused flow instabilities due to droplet merging, resulting in (F) varying crosslinking densities among microgels. (G) The final chip design supported stable flow and equal curing of all microgels, resulting in (H) monodisperse Dex-TA microgels. (I) Fluorescence confocal microscopy confirmed homogeneous crosslinking of microgels that were cured using the optimized $\mathrm{H}_{2} \mathrm{O}_{2}$ diffusion platform. Black scale bars depict $25 \mu \mathrm{m}$, white scale bars depict $250 \mu \mathrm{m}$, red scale bar depicts $100 \mu \mathrm{m}$. 

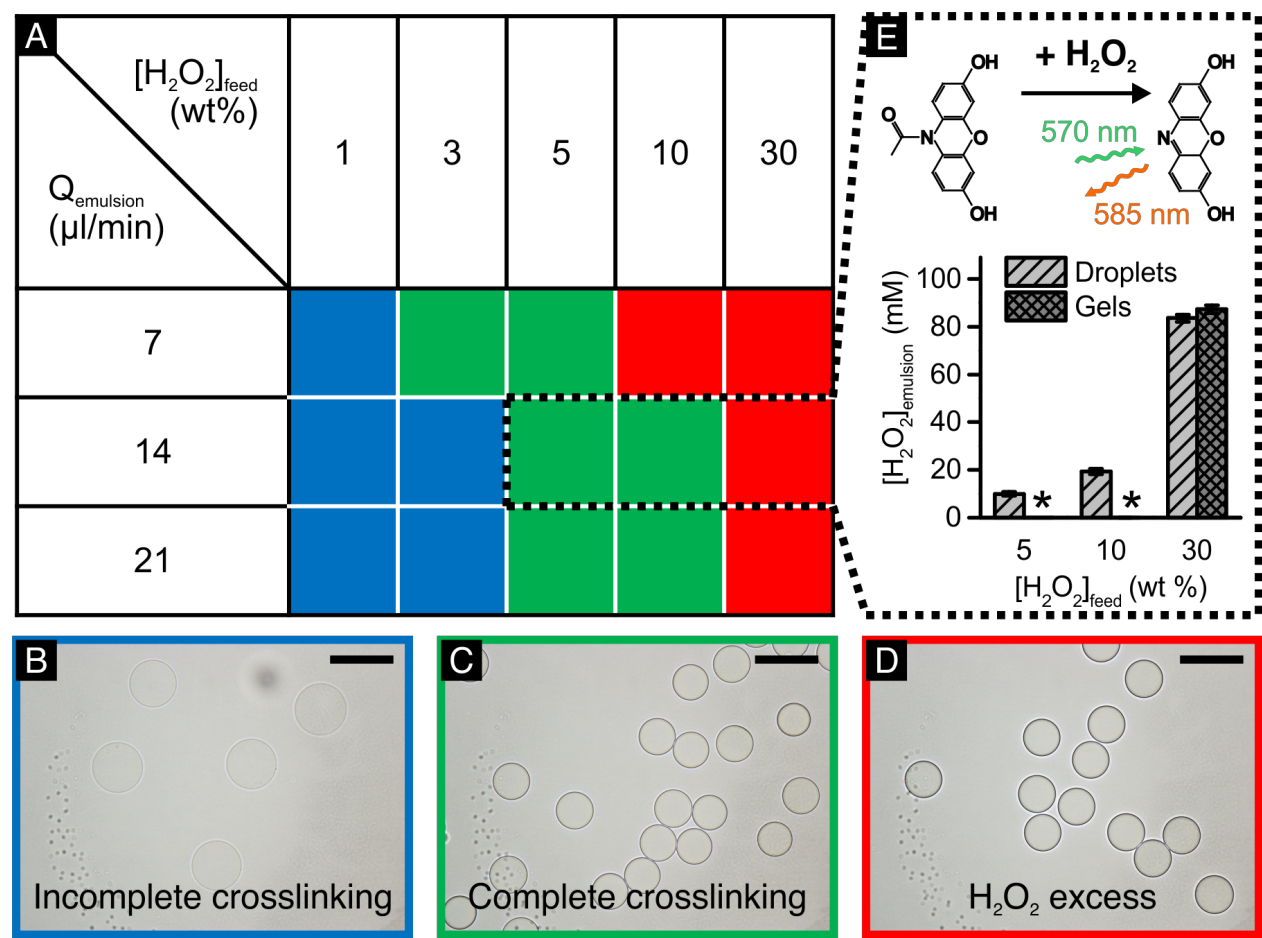

Figure 3. Determining functional crosslinking window for single cell microencapsulation. (A) Qualification of Dex-TA microgel crosslinking as function of the emulsion flow rate $\left(Q_{\text {emulsion }}\right)$ and concentration of the $\mathrm{H}_{2} \mathrm{O}_{2}$ feed. Blue, green, and red indicate (B) incomplete crosslinking, (C) complete crosslinking, and (D) $\mathrm{H}_{2} \mathrm{O}_{2}$ excess, respectively. (E) $\mathrm{H}_{2} \mathrm{O}_{2}$ quantification of residual $\mathrm{H}_{2} \mathrm{O}_{2}$ in Dex-TA microgel precursor droplet and crosslinked microgels. * Indicates 'not detectable'. Scale bars depict $50 \mu \mathrm{m}$.

\section{Tuning diffusion-based microgel production}

We then characterized the gelation platform to identify an optimal production window for cell encapsulation. Specifically, we aimed to produce completely crosslinked microgels without reaching cytotoxic levels of $\mathrm{H}_{2} \mathrm{O}_{2}$. As our system has fixed dimensions and constant diffusivity, Fick's law dictates that the amount of $\mathrm{H}_{2} \mathrm{O}_{2}$ that diffuses from the feed channel through the PDMS wall and oil into the microdroplets is determined by both the concentration difference, which approximates the $\mathrm{H}_{2} \mathrm{O}_{2}$ feed concentration $\left(\left[\mathrm{H}_{2} \mathrm{O}_{2}\right]_{\text {feed }}\right)$ and the diffusion time, which scales with the emulsion's flow rate $\left(Q_{\text {emulsion }}\right)$. The lower production limit of our gelation platform in terms of $\left[\mathrm{H}_{2} \mathrm{O}_{2}\right]_{\text {feed }}$ and $\mathrm{Q}_{\text {emulsion }}$ was determined by qualifying the amount of microgel swelling (Figure 3A). Incomplete crosslinking resulted in the absence of microgels (i.e. dissolved) or relatively large microgels 
with vague contours (i.e. swollen) as compared to completely crosslinked microgels (Figure 3B, C). Conversely, excessive $\mathrm{H}_{2} \mathrm{O}_{2}$ supplementation did not affect microgel morphology (Figure 3D). The upper production limit was determined by reaching the cytocompatible $\mathrm{H}_{2} \mathrm{O}_{2}$ limit, which was detected as residual $\mathrm{H}_{2} \mathrm{O}_{2}$ in microgels that had been crosslinked using increasing amounts of $\mathrm{H}_{2} \mathrm{O}_{2}$. Specifically, we quantified $\mathrm{H}_{2} \mathrm{O}_{2}$ in collected samples using the fluorescencebased substrate Amplex Red, which is oxidized by $\mathrm{H}_{2} \mathrm{O}_{2}$ to form highly fluorescent resorufin (Figure $3 E$ ) ${ }^{[29]}$. First, we validated this approach by measuring $\mathrm{H}_{2} \mathrm{O}_{2}$ concentrations in non-gelating microdroplets (i.e. without HRP). Indeed, increasing $\left[\mathrm{H}_{2} \mathrm{O}_{2}\right]_{\text {feed }}$ correlated with increased $\mathrm{H}_{2} \mathrm{O}_{2}$ concentrations in noncrosslinking HRP-free droplets. Subsequent quantification of gel precursor droplets that contained HRP revealed that only high $(\geq 10 \%)\left[\mathrm{H}_{2} \mathrm{O}_{2}\right]_{\text {feed }}$ resulted in detectable levels of residual $\mathrm{H}_{2} \mathrm{O}_{2}$ in the microgels. $\mathrm{As}_{2} \mathrm{H}_{2}$ can be cytotoxic, we considered $\left[\mathrm{H}_{2} \mathrm{O}_{2}\right]_{\text {feed }}=5 \%$ and $Q_{\text {emulsion }}=14 \mu \mathrm{l} / \mathrm{min}$ as optimal parameters for the production of well-crosslinked cytocompatible Dex-TA microgels.

\section{Centering individual cells in enzymatically crosslinkable microgels}

Throughout literature, droplet microfluidics has failed to completely encapsulate single cells in microgels smaller than $150 \mu \mathrm{m}$. We demonstrated that this is the result of coupled emulsification and crosslinking, which traps cells in the off-center position in which they are forced during droplet formation. Here, we present encapsulation of single cells into enzymatically crosslinkable microgels using a novel strategy, where emulsification and gelation are uncoupled. Uncoupling emulsification and crosslinking is enabled through our modular microfluidic $\mathrm{H}_{2} \mathrm{O}_{2}$ diffusion platform, which delays the initiation of the crosslinking of cell-laden microdroplets. We hypothesized that such delayed crosslinking allows droplet movement and deformation to center the cells inside the uncured droplets. To validate our hypothesis, we analyzed the position of single cells in non-crosslinking (i.e. without $\mathrm{H}_{2} \mathrm{O}_{2}$ feed) Dex-TA precursor droplets at three different positions along the modular microfluidic chip setup: 1) directly after the droplet generator; 2 ) at the start of the $\mathrm{H}_{2} \mathrm{O}_{2}$ diffusion chip; and 3) at the end of the $\mathrm{H}_{2} \mathrm{O}_{2}$ diffusion chip (Figure 4A). As observed before, cells were positioned at the droplets' oil/water interface immediately after encapsulation (Figure 4B). In contrast, by the end of the diffusion chip, most cells repositioned towards the center of microdroplets (Figure $4 \mathrm{C}$ ). Quantification of the cell 
position as measured by the distance between the cell's center and the microgel's center (Figure 4D) validated this observation, as indicated by the blue data points in Figure $4 \mathrm{E}$. Similar centering behavior was observed in gelating droplets (i.e. with $\mathrm{H}_{2} \mathrm{O}_{2}$ feed), as demonstrated by almost complete centering of single cells in Dex-TA microgels that were produced using this diffusion-based crosslinking method, as indicated by green data points in Figure 4E. Conversely, the conventional encapsulation approach, were emulsification and gelation are coupled, resulted in off-center positioning of cells in microgels. The cell position within these microgels did not significantly differ from the cell position at the droplet generator, confirming indicated by red data points in Figure 4E. 3D zstacking of fluorescence confocal images confirmed that cells were positioned in the center of Dex-TA microgels, indeed completely encapsulating all cells (Figure $4 F)$.

Lastly, Dex-TA microgels' short- and long-term cytocompatibility was assessed using a four week in vitro culture experiment with encapsulated MSCs (Figure 4G). Compared to non-encapsulated MSCs (i.e. syringe control), encapsulated MSCs were not affected by the microencapsulation procedure, indicating the mild and cytocompatible nature of the procedure. In fact, over 98 $\%$ of the cells were viable in both encapsulated and non-encapsulated samples on day 0 , as measured using a live/dead stain comprising calcein AM and ethidium homodimer-1 (EthD-1). Culturing individually encapsulated MSCs for up to four weeks also confirmed long-term cytocompatibility of the enzymatically crosslinked Dex-TA microenvironments. Remarkably, over $90 \%$ of cells remained alive (i.e. EthD-1 negative) of which $85 \%$ were metabolically active (i.e. calcein AM positive) after 28 days of in vitro culture (Figure $4 \mathrm{H}$ ). This makes the platform compatible with both short- and long-term in vitro studies such as fast drug metabolism screenings or more gradual stem cell differentiation assays. 

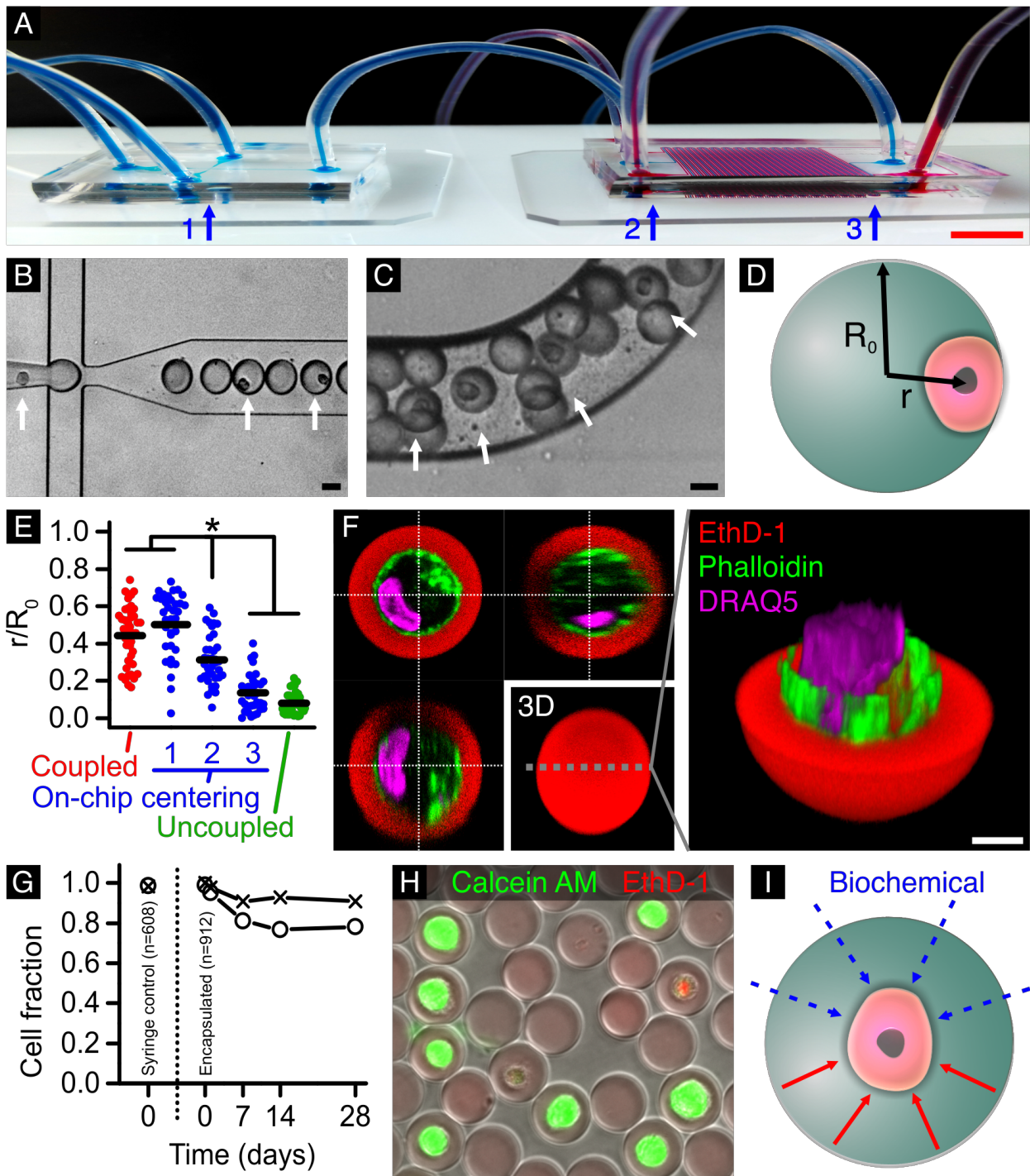

$\rightarrow$ EthD-1 neg. $-0-$ Calcein pos.
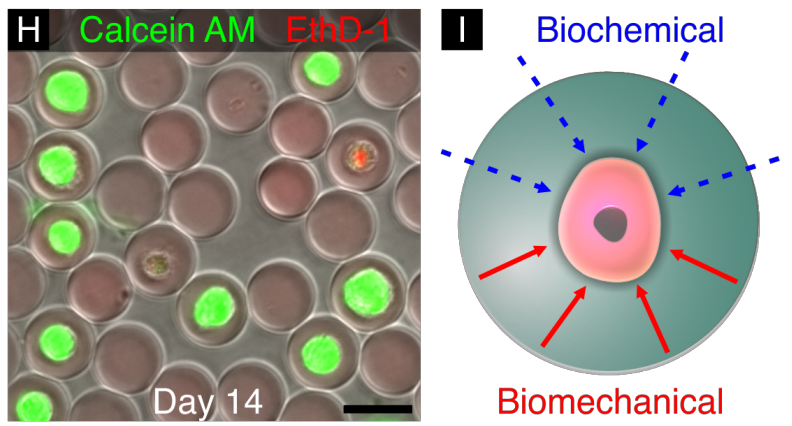

Figure 4. Centering single cells in microgels using the modular microfluidic $\mathrm{H}_{2} \mathrm{O}_{2}$ diffusion platform. (A) A standard microfluidic droplet generator connected in series to the $\mathrm{H}_{2} \mathrm{O}_{2}$ diffusion platform. The position of cells in microgel precursor droplets (white arrows) was analyzed (B) immediately after the droplet generator (1), at the start of the diffusion chip (2), and (C) at the end of the diffusion chip (3). (D, E) Cell position within microgels produced using a conventional system (i.e. coupled emulsification and gelation; red), and in gel precursor droplets (blue) and microgels (green) produced using our modular platform (i.e. uncoupled) was quantified by $r / R_{0}$, with $r$ the distance between the cell and microgel centers and $R_{0}$ the microgel radius; number 1 to 3 correspond with positions indicated in (A), * indicates significance with a $p<0,05 \quad(n=35)$. Enzymatically crosslinked Dex-TA microgels (F) completely encapsulated single MSCs, (G, H) 
remained intact, and had no detrimental effect on cell viability during long-term in vitro culture. (I) Schematic representation of uniform and symmetrical distributed biochemical and biomechanical stimuli acting on a single cell encapsulated in the center of a microgel. Black scale bars depict 25 $\mu \mathrm{m}$, white scale bars depict $5 \mu \mathrm{m}$, red scale bar depicts $5 \mathrm{~mm}$.

Cells encapsulated using the modular platform are located in the center of microgels. Rationally, these cells experience near uniform external biochemical and biomechanical stimuli (Figure 4I). Compared to off-center cell encapsulation, as observed with conventional enzyme-based microencapsulation, centered cells are provided with a better controlled microenvironment.

\section{Conclusion}

We reported on a novel microfluidic device that is readily compatible with a standard droplet generator to enable diffusion-based gelation of enzymatically crosslinkable microgels. Using the reported modular platform, we effectively uncoupled the emulsification and gelation of Dex-TA microgels. This approach effectively remedied the challenges of clogging and inhomogeneous crosslinking, which are typically observed with conventional microgel production platforms. Moreover, delayed crosslinking of single-cell-laden microdroplets resulted in centering of the cells, thereby preventing cell escape and ensuring uniform 3D culture conditions. Covalently crosslinked Dex-TA microgels remained intact and supported over $90 \%$ cell viability during long-term (28d) in vitro culture. Centering cells provides ultimate controlled over biochemical and biomechanical stimulation, which aids reproducible and reliable outcomes of single-cell-based studies. 


\section{Acknowledgements}

We thank Claas Willem Visser for discussions. We acknowledge A.J.S. Renard (Ziekenhuisgroep Twente) and the BIOS Lab-on-a-Chip group for providing biological samples and fluorocarbon oil, respectively.

\section{References}

[1] J. Leijten and A. Khademhosseini, "From Nano to Macro: Multiscale Materials for Improved Stem Cell Culturing and Analysis", Cell Stem Cell, vol. 18, pp. 20-4, 2016.

[2] N. A. Peppas, J. Z. Hilt, A. Khademhosseini, and R. Langer, "Hydrogels in biology and medicine: From molecular principles to bionanotechnology", Advanced Materials, vol. 18, pp. 1345-1360, 2006.

[3] K. Y. Lee and D. J. Mooney, "Hydrogels for tissue engineering", Chemical Reviews, vol. 101, pp. 1869-1879, 2001.

[4] D. S. W. Benoit, M. P. Schwartz, A. R. Durney, and K. S. Anseth, "Small functional groups for controlled differentiation of hydrogel-encapsulated human mesenchymal stem cells", Nat Mater, vol. 7, pp. 816-823, 2008.

[5] M. P. Lutolf, J. L. Lauer-Fields, H. G. Schmoekel, A. T. Metters, F. E. Weber, G. B. Fields, and J. A. Hubbell, "Synthetic matrix metalloproteinasesensitive hydrogels for the conduction of tissue regeneration: Engineering cell-invasion characteristics", Proceedings of the National Academy of Sciences of the United States of America, vol. 100, pp. 54135418, 2003.

[6] B. K. Mann, R. H. Schmedlen, and J. L. West, "Tethered-TGF-beta increases extracellular matrix production of vascular smooth muscle cells", Biomaterials, vol. 22, pp. 439-44, 2001.

[7] J. Leijten, Y. C. Chai, I. Papantoniou, L. Geris, J. Schrooten, and F. P. Luyten, "Cell based advanced therapeutic medicinal products for bone repair: Keep it simple?", Adv Drug Deliv Rev, vol. 84, pp. 30-44, 2015.

[8] J. H. Sung and M. L. Shuler, "A micro cell culture analog (mu CCA) with 3$D$ hydrogel culture of multiple cell lines to assess metabolism-dependent cytotoxicity of anti-cancer drugs", Lab on a Chip, vol. 9, pp. 1385-1394, 2009.

[9] L. M. Bian, M. Guvendiren, R. L. Mauck, and J. A. Burdick, "Hydrogels that mimic developmentally relevant matrix and $\mathrm{N}$-cadherin interactions enhance MSC chondrogenesis", Proceedings of the National Academy of Sciences of the United States of America, vol. 110, pp. 10117-10122, 2013.

[10] A. Ranga, S. Gobaa, Y. Okawa, K. Mosiewicz, A. Negro, and M. P. Lutolf, "3D niche microarrays for systems-level analyses of cell fate", Nature Communications, vol. 5, p. 4324, 2014. 
[11] J. S. Liu and Z. J. Gartner, "Directing the assembly of spatially organized multicomponent tissues from the bottom up", Trends Cell Biol, vol. 22, pp. 683-91, 2012.

[12] D. Velasco, E. Tumarkin, and E. Kumacheva, "Microfluidic Encapsulation of Cells in Polymer Microgels", Small, vol. 8, pp. 1633-1642, 2012.

[13] Y. Ma, M. P. Neubauer, J. Thiele, A. Fery, and W. T. S. Huck, "Artificial microniches for probing mesenchymal stem cell fate in 3D", Biomaterials Science, vol. 2, pp. 1661-1671, 2014.

[14] A. Kumachev, J. Greener, E. Tumarkin, E. Eiser, P. W. Zandstra, and E. Kumacheva, "High-throughput generation of hydrogel microbeads with varying elasticity for cell encapsulation", Biomaterials, vol. 32, pp. 147783, 2011.

[15] T. Xu, H. Kincaid, A. Atala, and J. J. Yoo, "High-Throughput Production of Single-Cell Microparticles Using an Inkjet Printing Technology", Journal of Manufacturing Science and Engineering, vol. 130, pp. 021017-021017, 2008.

[16] S. Utech, R. Prodanovic, A. S. Mao, R. Ostafe, D. J. Mooney, and D. A. Weitz, "Microfluidic Generation of Monodisperse, Structurally Homogeneous Alginate Microgels for Cell Encapsulation and 3D Cell Culture", Adv Healthc Mater, vol. 4, pp. 1628-33, 2015.

[17] S. Allazetta, L. Kolb, S. Zerbib, J. Bardy, and M. P. Lutolf, "Cell-Instructive Microgels with Tailor-Made Physicochemical Properties", Small, vol. 11, pp. 5647-56, 2015.

[18] P. Hematti, J. Kim, A. P. Stein, and D. Kaufman, "Potential role of mesenchymal stromal cells in pancreatic islet transplantation", Transplantation Reviews, vol. 27, pp. 21-29, 2013.

[19] R. Novak, Y. Zeng, J. Shuga, G. Venugopalan, D. A. Fletcher, M. T. Smith, and R. A. Mathies, "Single-cell multiplex gene detection and sequencing with microfluidically generated agarose emulsions", Angew Chem Int Ed Engl, vol. 50, pp. 390-5, 2011.

[20] S. V. Bhujbal, B. de Haan, S. P. Niclou, and P. de Vos, "A novel multilayer immunoisolating encapsulation system overcoming protrusion of cells", Sci Rep, vol. 4, p. 6856, 2014.

[21] S. Akbari and T. Pirbodaghi, "Microfluidic encapsulation of cells in alginate particles via an improved internal gelation approach", Microfluidics and Nanofluidics, vol. 16, pp. 773-777, 2014.

[22] W. H. Tan and S. Takeuchi, "Monodisperse Alginate Hydrogel Microbeads for Cell Encapsulation", Advanced Materials, vol. 19, pp. 2696-2701, 2007.

[23] L. S. Teixeira, J. Feijen, C. A. van Blitterswijk, P. J. Dijkstra, and M. Karperien, "Enzyme-catalyzed crosslinkable hydrogels: emerging 
strategies for tissue engineering", Biomaterials, vol. 33, pp. 1281-90, 2012.

[24] S. Henke, J. Leijten, E. Kemna, M. Neubauer, A. Fery, A. van den Berg, A. van Apeldoorn, and M. Karperien, "Enzymatic Crosslinking of Polymer Conjugates is Superior over Ionic or UV Crosslinking for the On-Chip Production of Cell-Laden Microgels", Macromol Biosci, 2016.

[25] C. W. Yung, L. Q. Wu, J. A. Tullman, G. F. Payne, W. E. Bentley, and T. A. Barbari, "Transglutaminase crosslinked gelatin as a tissue engineering scaffold", J Biomed Mater Res A, vol. 83, pp. 1039-46, 2007.

[26] T. A. Ahmed, E. V. Dare, and M. Hincke, "Fibrin: a versatile scaffold for tissue engineering applications", Tissue Eng Part B Rev, vol. 14, pp. 199215, 2008.

[27] R. Jin, C. Hiemstra, Z. Y. Zhong, and J. Feijen, "Enzyme-mediated fast in situ formation of hydrogels from dextran-tyramine conjugates", Biomaterials, vol. 28, pp. 2791-2800, 2007.

[28] S. K. Both, A. J. van der Muijsenberg, C. A. van Blitterswijk, J. de Boer, and J. D. de Bruijn, "A rapid and efficient method for expansion of human mesenchymal stem cells", Tissue Engineering, vol. 13, pp. 3-9, 2007.

[29] J. G. Mohanty, J. S. Jaffe, E. S. Schulman, and D. G. Raible, "A highly sensitive fluorescent micro-assay of $\mathrm{H} 2 \mathrm{O} 2$ release from activated human leukocytes using a dihydroxyphenoxazine derivative", Journal of Immunological Methods, vol. 202, pp. 133-141, 1997. 
Chapter 7

General discussion 


\section{Aim}

The general aim of this thesis is the development of novel microencapsulation strategies to improve the transplantation of insulin producing islets of Langerhans or stem cell derived beta cells. Current encapsulation strategies focus mostly on protecting the encapsulated islets of Langerhans or beta cells from the immune system. However, the successful function of the encapsulated cells is of highest importance, and thus the main focus point in this thesis. Classically, islet encapsulation is done using droplet generators to produce capsules that encapsulate one or more islets, and thus are relatively big, often with a diameter $>300 \mu \mathrm{m}$, and with a thick protective layer. This is effective for immunoprotection, but causes beta cells to be too far away from blood vessels. This hampers nutrient and oxygen supply and causes delayed insulin response. This latter aspect might be particularly relevant to islets, as their native blood supply is very high, with $10 \%$ of the pancreatic blood supply going to the islets, while only contributing for $1-2 \%$ of the pancreas mass ${ }^{[1]}$. A potential strategy to overcome this diffusion limitation problem is scaling down the size of the capsules. This scaling down requires specific technology. In this thesis we explored the use of microfluidics to produce such small capsules.

A limited number of materials is currently used for islet encapsulation. The polysaccharide alginate and the synthetic polymer polyethylene-glycol (PEG) are most used to obtain immunoprotective capsules. These materials have the disadvantage that they are often bioinert. This is at one hand advantageous since it helps in protecting the capsule against attacks from the immune system, but at the other hand is also a drawback. They also do not possess functionality towards the encapsulated cells by for example supporting the cells in their function. Furthermore functional integration of these capsules in the body upon implantation is also compromised. Hence, new materials are needed that, next to the protection against the immune system, can support the encapsulated cells and facilitate integration in the recipients' body. In this thesis a new material platform using enzymatically crosslinked polymer conjugates is studied for the encapsulation of insulin producing beta cells. 


\section{Cells sources}

The number of patients with type 1 diabetes that can undergo beta cell replacement or islet transplantation is limited by the availability of donor tissue and hindered by the need for immunosuppression. Often two or three donors are needed to treat one patient. Thus there is a need for more optimal use of the currently available donors. If one donor would be enough to treat one patient, this will already increase the number of patients that can be treated substantially. During an islet isolation procedure, there is a lot of discarded tissue that consists of fragmented islets and tissue with few beta cells. When the fragmented islets and loose beta cells could be purified, they might be effectively put together in core-shell microgels, as presented in chapter 5 , and directly co-transplanted with the islets in order to transplant as much beta cell mass as possible. Next to this, the islet depleted tissue, which is partially duct tissue, is expected to contain beta cell progenitors ${ }^{[2-4]}$. Aggregation of the duct cells is an essential step in the differentiation process ${ }^{[2]}$ and could potentially be improved using specially tailored core-shell gels as described in chapter 5 of this thesis.

The ultimate type 1 diabetes treatment, however, would use a replenishable beta cell source of autologous origin, that doesn't require immunosuppression and is widely available. This source of the future could be beta cells derived from induced pluripotent stem cells (iPSC). With the potential transplantation of pluripotent stem cell derived beta cells there is an inherent risk of uncontrolled proliferation of a (sub)population of stem cells that that did not differentiate. Thus there is the need of strategies to minimize this risk. We have shown in chapter 4 that microgels can limit the proliferation of encapsulated cells. This opens the possibility to further develop this proliferation restrictive technology towards the transplantation of stem cell derived beta cells, where there is the risk of uncontrolled proliferation after transplantation ${ }^{[5]}$. Such strategy could be combined with chemical strategies that can ablate transplanted iPSCs at any time in the body ${ }^{[6]}$. Further development of the core-shell technology will have to focus at one hand on long term proliferation restriction and confinement of the cells in the gels, and at the other on supporting or boosting the, in case of beta cells, response of insulin secretion of the encapsulated cells. 


\section{Tissue engineering}

The way of transplanting islets of Langerhans or cells in general is a challenge. Islets are nowadays transplanted in the liver via infusion through the portal vein, but this has numerous problems. A general problem when transplanting in whole blood is the instant blood-mediated inflammatory reaction (IBMR) ${ }^{[7]}$. For islets transplanted via the portal vein in the liver, there is the problem of the liver itself. The liver is a hostile environment with low oxygen tension and high toxin levels, leading to suboptimal function and death of the transplanted islets ${ }^{[8]}$. Thus there is need for alternative transplantation sites which don't have these disadvantages ${ }^{[8]}$. As most alternative sites don't have a structure that allows for bare islets infusion like the liver vasculature, there will be some form of biomaterial necessary in which the islets can be transplanted.

In our department Buitinga et al. have developed a successful microwell device to transplant islets of Langerhans ${ }^{[9,10]}$. Both the solid gels developed in chapter 2, 3 and 4, and the core-shell gels developed in chapter 5 will fit in this system, and thus could be employed to transplant beta cells using these microwell devices. Another very promising strategy would be incorporating the microgels in a 3D printing strategy. 3D printing for tissue engineering is very popular topic of research, as it is possible to print living cells in combination with biomaterials in a structure of choice. Our group has demonstrated the 3D printing of small microgels ${ }^{[11]}$ and islets of Langerhans ${ }^{[12]}$ in hydrogel material. As the size of the microgels in this thesis lies between the small microgels and islets of Langerhans, the cell-laden microgels presented in this thesis can also be printed to create a tissue engineering construct.

\section{General relevancy for tissue engineering}

The building blocks for tissue engineering strategies developed for the transplantation of beta cells are also generally applicable for (bottom-up) tissue engineering purposes by changing to other cell types. To illustrate this, in chapter 3 we successfully produced MSC encapsulating microgels that could serve as functional building blocks in the fields of bone and fat tissue engineering. With our microgel technology using enzymatic crosslinking, we can easily vary the composition of the microgels by varying in polymer conjugates, and vary the mechanical properties by varying the polymer concentration. Thus with this 
technology we can optimize the microenvironment for specific applications. The building blocks could be incorporated in all kinds of microfabrication technologies for (bottom-up) tissue engineering techniques like 3D printing and micromolding. This enables the addition of a layer of complexity in the construction of spatially organized complex tissues ${ }^{[13]}$. Furthermore, embedding cells first in microgels before they are employed in bioprinting processes may help in protecting the cells from shear forces during printing. These forces are responsible for substantial cell death during cell-biomaterial deposition.

Fast and adequate vascularization is essential for a tissue engineering construct to be successful. Cells need oxygen and nutrients, and waste products have to be removed. As soon as a construct is implanted, there is the need of vascularization to do this. Using a modular tissue engineering construct, it is relatively easy to engineer empty spaces between the building blocks. This can be achieved, for example, by packing microgel spheres. In between the round spheres, there will be automatically an interconnected open network that could facilitate the ingrowth of blood vessels. In chapter 4 we have found evidence supporting this. In just a two week time period, red blood cells containing blood vessel had invaded packed gels in a transplantation experiment in which the constructs were injected under the kidney capsule. The empty space could also be filled with a dedicated angiogenesis promoting hydrogel before transplantation ${ }^{[14]}$. Combination of the microgels with other cells that aid in angiogenesis, like endothelial progenitors, can potentially even further increase this potential ${ }^{[15,16]}$.

\section{Other areas of application of microgels}

As we have shown in chapter 2, 3, 5 and 6, mesenchymal stem cells can be encapsulated and maintained in microgels with very high survival. This gives the opportunity to use microgels with encapsulated MSCs as "living factories" for the delivery of trophic factors, for example to enhance cartilage repair, as MSCs are known to improve cartilage matrix production ${ }^{[17]}$. Additionally, infused MSCs are believed to have therapeutic value in numerous diseases, although the exact mechanisms are still unknown ${ }^{[18]}$. In most cases the beneficial effect is attributed to the secretion of trophic factors. Microencapsulation of MSCs might enhance or prolong this by stimulating and/or protecting the MSCs ${ }^{[19]}$. In case of allogeneic 
MSCs this is most intuitive, since protection by encapsulation of the MSCs from attack by the immune cells is expected to prolong their residence time in the body and thus will increase the time they can secrete trophic factors. Next to this, encapsulation in a dedicated matrix, potentially enriched with growth factors, could stimulate the MSCs to produce more therapeutic trophic factors.

Besides use for therapeutic purposes, microgels are also a very promising tool in disease modeling and high throughput screening. High throughput screening is very important for drug discovery and disease studies. Usually large numbers of microwell plates with 2D cell cultures are used. Increasingly 3D cultures in the form of cell aggregates are used to better mimic the in vivo situation. Microgel technology can potentially hugely improve the high throughput screening process by making the model more relevant by reproducibly encapsulating cells in a relevant matrix, which will make their response more close to the in vivo physiological situation ${ }^{[2,21]}$. This could be both in solid gels as presented in chapter 2, 3, 4 and 6, as in core-shell gels presented in chapter 5.

\section{Further development of microgel technology}

Although cell encapsulating microgel technology dates back to alginate encapsulation of islets of Langerhans in $1980^{[22]}$, microfluidic cell encapsulation only took off around the first decade in the $21^{\text {st }}$ century. In principle, all handlings that can be done on normal sized hydrogels can also be applied on microgels with little adaption. Due to their extremely small size, all kinds of additional modifications are more easily applicable to microgels. Especially modifications that rely on diffusion into the crosslinked hydrogel, as diffusion times into the hydrogel core increase rapidly with increasing hydrogel size. So diffusion into the entire microgel is faster than into a large gel, which makes it possible to functionalize microgels with functional peptides post-crosslinking using biotinstreptavidin, as we show in Kamperman et al. (publication in preparation) ${ }^{[23]}$.

Next to decorating the hydrogels with biological cues like peptides, the small size of microgels opens the possibility to tune the stiffness of microgels dynamically, after the hydrogel is formed, as shown by Kamperman et al. (publication in preparation) ${ }^{[24]}$. Using this technique the stiffness of microgels can be changed in a given point in time. This is a very exciting research tool to 
investigate the effects of (changing) stiffness on the differentiation and function of cells. As differentiation is known to be influenced by stiffness [25], and complicated stem cell differentiation protocols tend to consist of several steps with varying media compositions ${ }^{[26]}$, these differentiation protocols might be enhanced by steering the stiffness during differentiation.

\section{Conclusion}

In this thesis we presented microfluidic techniques for the encapsulation of (stem cell derived) beta cells and mesenchymal stromal cells into specialized microgels of enzymatically crosslinked tyramine conjugated polymers with tunable stiffness and composition. Both solid and core-shell microgels were created with a broad range of potential applications, from a proliferation restricting microenvironment to a microbioreactor. These microgels are a promising research tool in amongst others drug screening and cell differentiation studies, and are a step towards transplantation of functionally encapsulated (beta) cells, alone or in tissue engineered constructs. 


\section{References}

[1] N. Yaginuma, T. Takahashi, K. Saito, and M. Kyoguku, "The microvasculature of the human pancreas and its relation to Langerhans islets and lobules.", in The Pancreas: Biology, Pathobiology, and Disease, Second Edition, 1993, p. 760.

[2] S. Yatoh, R. Akashi, T. Omer, and A. Sharma, "Differentiation of AffinityPurified Human Pancreatic Duct Cells to-Cells", Diabetes, vol. 56, no. 7, pp. 1802-1809, 2007.

[3] P. A. Lysy, G. C. Weir, and S. Bonner-Weir, "Making $\beta$ cells from adult cells within the pancreas", Current Diabetes Reports, vol. 13, no. 5, pp. 695703, 2013.

[4] E. Corritore, E. Dugnani, V. Pasquale, R. Misawa, P. Witkowski, J. Lei, J. Markmann, L. Piemonti, E. M. Sokal, S. Bonner-Weir, and P. A. Lysy, “ $\beta$-Cell differentiation of human pancreatic duct-derived cells after in vitro expansion.", Cellular reprogramming, vol. 16, no. 6, pp. 456-66, 2014.

[5] S. G. Hong, T. Winkler, C. Wu, V. Guo, S. Pittaluga, A. Nicolae, R. E. Donahue, M. E. Metzger, S. D. Price, N. Uchida, S. A. Kuznetsov, T. Kilts, L. Li, P. G. Robey, and C. E. Dunbar, "Path to the clinic: assessment of iPSCbased cell therapies in vivo in a nonhuman primate model.", Cell reports, vol. 7, no. 4, pp. 1298-309, 2014.

[6] S. Yagyu, V. Hoyos, F. Del Bufalo, and M. K. Brenner, "Multiple mechanisms determine the sensitivity of human-induced pluripotent stem cells to the inducible caspase-9 safety switch", Molecular Therapy - Methods \& Clinical Development, vol. 3, no. October 2015, p. 16003, 2016.

[7] W. Bennet, B. Sundberg, C. G. Groth, M. D. Brendel, D. Brandhorst, H. Brandhorst, R. G. Bretzel, G. Elgue, R. Larsson, B. Nilsson, and O. Korsgren, "Incompatibility between human blood and isolated islets of Langerhans: a finding with implications for clinical intraportal islet transplantation?", Diabetes, vol. 48, no. 10, pp. 1907-1914, 1999.

[8] A. Rajab, "Islet transplantation: Alternative sites", Current Diabetes Reports, vol. 10, no. 5, pp. 332-337, 2010.

[9] M. Buitinga, R. Truckenmüller, M. A. Engelse, L. Moroni, H. W. M. Ten Hoopen, C. A. van Blitterswijk, E. J. P. de Koning, A. A. van Apeldoorn, and M. Karperien, "Microwell Scaffolds for the Extrahepatic Transplantation of Islets of Langerhans", PLOS ONE, vol. 8, no. 5, pp. 1-11, 2013.

[10] M. Buitinga, "Plan- $\beta$ : a bioengineering approach against type 1 diabetes", phdthesis, Universiteit Twente, Enschede, 2015.

[11] T. Kamperman, S. Henke, A. van den Berg, S. R. Shin, A. Tamayol, A. Khademhosseini, M. Karperien, and J. Leijten, "Single Cell Microgel Based Modular Bioinks for Uncoupled Cellular Micro- and Macroenvironments", In Preparation. 
[12] G. Marchioli, L. van Gurp, P. P. van Krieken, D. Stamatialis, M. Engelse, C. A. van Blitterswijk, M. B. J. Karperien, E. de Koning, J. Alblas, L. Moroni, and A. A. van Apeldoorn, "Fabrication of three-dimensional bioplotted hydrogel scaffolds for islets of Langerhans transplantation.", Biofabrication, vol. 7, no. 2, p. 25009, 2015.

[13] J. Leijten, J. Rouwkema, Y. S. Zhang, A. Nasajpour, M. R. Dokmeci, and A. Khademhosseini, "Advancing Tissue Engineering: A Tale of Nano-, Micro-, and Macroscale Integration", Small, vol. 12, no. 16, pp. 2130-2145, 2016.

[14] J. A. Burdick, R. L. Mauck, and S. Gerecht, "To Serve and Protect: Hydrogels to Improve Stem Cell-Based Therapies", Cell Stem Cell, vol. 18, no. 1, pp. 13-15, 2016.

[15] M. Buitinga, K. Janeczek Portalska, D.-J. Cornelissen, J. Plass, M. Hanegraaf, F. Carlotti, E. de Koning, M. Engelse, C. van Blitterswijk, M. Karperien, A. van Apeldoorn, and J. de Boer, "Coculturing Human Islets with Proangiogenic Support Cells to Improve Islet Revascularization at the Subcutaneous Transplantation Site", Tissue Engineering Part A, vol. 22, no. 3-4, pp. 375-385, 2016.

[16] D. Hyaluronic, A. Hydrogels, K. J. Portalska, L. M. Teixeira, J. C. H. Leijten, R. Jin, C. Van Blitterswijk, J. De Boer, and M. Karperien, "Boosting Angiogenesis and Functional Vascularization by Endothelial-Like Mesenchymal Stromal Cells", vol. 20, 2014.

[17] L. Wu, J. C. Leijten, N. Georgi, J. N. Post, C. A. van Blitterswijk, and M. Karperien, "Trophic effects of mesenchymal stem cells increase chondrocyte proliferation and matrix formation", Tissue engineering. Part A, vol. 17, no. 9-10, pp. 1425-1436, 2011.

[18] J. Ankrum and J. M. Karp, "Mesenchymal stem cell therapy: Two steps forward, one step back", Trends in Molecular Medicine, vol. 16, no. 5, pp. 203-209, 2010.

[19] A. Moshaverinia, C. Chen, X. Xu, S. Ansari, H. H. Zadeh, S. R. Schricker, M. L. Paine, J. Moradian-Oldak, A. Khademhosseini, M. L. Snead, and S. Shi, "Regulation of the stem cell-host immune system interplay using hydrogel coencapsulation system with an anti-inflammatory drug", Advanced Functional Materials, vol. 25, no. 15, pp. 2296-2307, 2015.

[20] S. Allazetta and M. P. Lutolf, "Stem cell niche engineering through droplet microfluidics.", Current opinion in biotechnology, vol. 35, pp. 86-93, 2015.

[21] F. Hofstaedter and R. Ebner, "The Use of 3-D Cultures for High-Throughput Screening ":, Journal of biomolecular screening, vol. 9, no. 4, pp. 273-285, 2004.

[22] F. Lim and A. Sun, "Microencapsulated islets as bioartificial endocrine pancreas", Science, vol. 210, no. 4472, pp. 908-910, 1980.

[23] T. Kamperman, S. Henke, C. Kelder, M. Koerselman, P. J. Dijkstra, J. Leijten, 
and M. Karperien, "In Situ Modifiable Multifunctional Hydrogel for Advanced Tissue Engineering", In Preparation.

[24] T. Kamperman, S. Henke, M. Neubauer, A. Fery, J. Leijten, and M. Karperien, "Engineering 3D Single Stem Cell Microniches With Dynamically Tunable Stiffness", In Preparation.

[25] W. L. Murphy, T. C. McDevitt, and A. J. Engler, "Materials as stem cell regulators", Nature Materials, vol. 13, no. 6, pp. 547-557, 2014.

[26] F. W. Pagliuca, J. R. Millman, M. Gürtler, M. Segel, A. Van Dervort, J. H. Ryu, Q. P. Peterson, D. Greiner, and D. A. Melton, "Generation of Functional Human Pancreatic $\beta$ Cells In Vitro", Cell, vol. 159, no. 2, pp. 428-439, 2014. 


\section{Summary}

In this thesis we describe the development and testing of microfluidic cell encapsulation strategies to improve the transplantation of (beta) cells. We started with a comparison of cells incapsulated in microgels made of three different materials. The cells were encapsulated in photo crosslinked polyethylene-glycol di-acrylate (PEGDA), ionic crosslinked alginate and enzymatic crosslinked dextran-tyramine (Dex-TA) microgels. We concluded that Dex-TA microgels proved to be superior over photo crosslinked PEGDA and ionic crosslinked alginate in terms of stability, mechanical properties, cells survival and metabolic activity (chapter $\mathbf{2}$ ). We thus continued with enzymatically crosslinked Dex-TA based gels for the rest of the research.

As Dex-TA hydrogel is bioinert, we decided to improve the bioactivity of the microgels by the addition of hyaluronic acid-tyramine. In the meantime we also product gels with different stiffnesses, resulting in soft gels with an Emodulus of approximately $3 \mathrm{kPa}$ of both pure Dex and the combination DexHA, and stiff gels of approximately $20 \mathrm{kPa}$ of both pure Dex and the combination DexHA (chapter 3). With differentiation of encapsulated hMSCs, soft gels proved to be superior for adipogenic differentiation of hMSCs and stiffer gels favored osteogenic differentiation. Interestingly, hyaluronic acid blocked the differentiation towards the osteogenic lineage, and did not seem to influence adipogenic differentiation. This finding warrants further research towards the mechanism of blocking or inhibiting the osteogenesis of hMSCs by hyaluronic acid.

The platform of soft and stiff and bioinert and bioactive microgels from chapter $\mathbf{3}$ was applied to create a proliferation restrictive microenvironment for stem cell derived beta cells, where there is the risk of unrestricted proliferation (chapter 4). To this end we used a beta cell model of MIN6 cells, a fast proliferating beta cell line, and evaluated the function, survival and proliferation of encapsulated cells. Stiff microgels were necessary to counteract unrestricted proliferation. Further research has to provide the exact optimal gel composition to balance long term stability and the ability of the cells to proliferate.

Next to the solid cell encapsulating microgels, we developed a platform to produce core-shell microgels using $\mathrm{H}_{2} \mathrm{O}_{2}$ supplied via diffusion for the 
crosslinking (chapter 5). These microgels have a crosslinked shell and a fluid core, in which cells can freely move and aggregate. Encapsulated cells successfully aggregated within one day both in vitro and in an ex vivo implantation model. This makes these gels a step forward from making aggregates using a microwell system, and a very interesting tool for high throughput screening and stem cell differentiation.

Again based on the $\mathrm{H}_{2} \mathrm{O}_{2}$ diffusion, we developed a platform to produce single cell encapsulating microgels smaller than $50 \mu \mathrm{m}$ (chapter 6). This platform enables the production of cell encapsulating sub $50 \mu \mathrm{m}$ enzymatically crosslinked microgels with high cell viability. The cells are centered in the gel, unlike most single cell microgels. Using these extremely small gels a tissue relevant packing density of single encapsulated cells can be reached in a tissue engineering construct, which is impossible using larger single cell gels. 


\section{Samenvatting}

In dit proefschrift wordt mijn onderzoek naar microflüdische technieken beschreven om cellen te vangen in hydrogel, ter verbetering van de transplantatie van (beta) cellen. Allereerst hebben we cellen die in microgelen zijn ingekapseld, vergeleken. Deze microgelen waren gemaakt van verschillende materialen. De cellen waren ingekapseld in, door middel van licht, gecrosslinkte (gebonden) polyethylene-glycol di-acrylate (PEGDA), ionisch gecrosslinkte alginaat en enzymatisch gecrosslinkte dextraan-tyramine (Dex-TA). De conclusie was dat Dex-TA microgelen het beste waren op het gebied van stabiliteit, mechanische eigenschappen, cel overleving en metabolische activiteit van de cellen (hoofdstuk 2). In de rest van het onderzoek zijn we daarom verder gegaan met gelen gebaseerd op enzymatisch gecrosslinkte Dex-TA.

Aangezien Dex-TA hydrogel bio-inert is, hebben we ter verbetering van de bioactiviteit hyaluronzuur-tyramine (HA-TA) toegevoegd aan de microgel. Dit resulteerde in DexHA hydrogelen. Tegelijkertijd hebben we ook hydrogelen met verschillende stijfheden gemaakt, resulterend in zachte gelen met een E-modulus van ongeveer $3 \mathrm{kPa}$ van zowel puur Dex als DexHA, en stijve gelen van ongeveer $20 \mathrm{kPa}$, ook van zowel puur Dex als DexHA (hoofdstuk 3). Tijdens de differentiatie van ingekapselde mesenchymale stamcellen (hMSCs) bleken de zachte gelen beter voor de adipogene (vet) richting en de stijve gelen beter voor de osteogene (bot) richting. Interessant was dat hyaluronzuur de differentiatie naar de osteogene richting blokkeerde, maar de adipogene differentiatie niet leek te beïnvloeden. Deze interessante vindingen zouden gebruikt kunnen worden in bijvoorbeeld kraakbeen herstel therapieën waar geen bot gevormd mag worden, en moeten daar voor verder onderzocht worden.

De techniek zoals beschreven in hoofdstuk 3 voor zachte en stijve, bioinerte en bioactive microgelen werd vervolgens toegepast om een celdeling (proliferatie) restrictieve micro-omgeving voor beta-cellen afkomstig van stamcellen te maken, aangezien hierbij risico is op ongecontroleerde celdeling hetgeen kan leiden tot een gezwel (hoofdstuk 4). Hiervoor hebben we als betacel model de snel delende MIN6 cellen gebruikt. De functie, overleving en proliferatie van ingekapselde cellen werd bestudeerd. Hierbij concludeerden wij dat stijve microgelen nodig zijn om de ongecontroleerde proliferatie tegen te 
gaan. Er is echter verder onderzoek nodig om tot een optimale gel samenstelling te komen die een goede balans biedt tussen stabiliteit op de lange termijn en de mogelijkheid tot celdeling van de ingekapselde cellen.

Naast de solide gelen met ingekapselde cellen hebben we ook een techniek ontwikkeld voor de productie van kern-schil microgelen, gebruikmakend van $\mathrm{H}_{2} \mathrm{O}_{2}$ diffusie voor de crosslinking (hoofdstuk 5). Deze microgelen hebben een gecrosslinkte schil en een vloeibare kern, waarin de cellen vrijuit kunnen bewegen en aggregeren (samenklonteren). Ingekapselde cellen aggregeerden inderdaad binnen één dag, zowel in vitro als in een ex vivo implantatiemodel. Hierdoor vormen deze hydrogelen een vooruitgang bij het maken van microaggregaten ten opzichte van een microwell systeem, en zijn deze gelen een zeer interessante tool voor het gebruik in "high throughput screening" en bij de differentiatie van stamcellen.

We hebben ook een techniek ontwikkeld om een enkele cel in te vangen in een microgel nauwelijks groter dan de cel zelf, eveneens gebaseerd op $\mathrm{H}_{2} \mathrm{O}_{2}$ diffusie voor de enzymatische crosslinking (hoofdstuk 6). In tegenstelling tot veel andere systemen met een enkele cel per gel, zitten de cellen in dit systeem gecentreerd in de gel. Met deze zeer kleine gelen kan een, voor weefsel relevante, celdichtheid gehaald worden in een kunstmatig opgebouwd weefsel, iets wat onmogelijk is bij gebruik van grotere gelen. 


\section{List of Publications}

\section{Peer-reviewed papers}

Sieger Henke, Jeroen Leijten, Evelien Kemna, Martin Neubauer, Andreas Fery, Albert van den Berg, Aart van Apeldoorn, Marcel Karperien. Enzymatic Crosslinking of Polymer Conjugates is Superior over lonic or UV Crosslinking for the On-Chip Production of Cell-Laden Microgels. Published in Macromolecular Bioscience, vol. 16, 2016

Jan Hendriks, Claas Willem Visser, Sieger Henke, Jeroen Leijten, Daniël B.F. Saris, Chao Sun, Detlef Lohse, Marcel Karperien. Optimizing cell viability in dropletbased cell deposition. Published in Nature Scientific Reports, vol. 5, 2015

Tom Kamperman, Sieger Henke, Albert van den Berg, Su Ryon Shin, Ali Tamayol, Ali Khademhosseini, Marcel Karperien, Jeroen Leijten. Single Cell Microgel Based Modular Bioinks for Uncoupled Cellular Micro- and Macroenvironments. Submitted

Milou Groot Nibbelink, Katarzyna Skrzypek, Lisanne Karbaat, Sanne Both, Jacqueline Plass, Bettie Klomphaar, Jéré van Lente, Sieger Henke, Marcel Karperien, Dimitrios Stamatialis, Aart van Apeldoorn. Mesenchymal stem cells and micropatterns of poly(ether sulfone) / poly(vinylpyrrolidon) membranes accelerates vascularization. Submitted

Sieger Henke, Tom Kamperman, Rong Wang, Pieter J Dijkstra, Jeroen Leijten, Marcel Karperien. The stiffness and composition of microhydrogels independently control mesenchymal stromal cell differentiation in the osteogenic lineage. In preparation

Sieger Henke, Jeroen Leijten, Tom Kamperman, Milou Groot Nibbelink, Rong Wang, Jacqueline Plass, Annemieke Tons, Maaike Hanegraaf, Francoise Carlotti, Eelco de Koning, Pieter J Dijkstra, Aart van Apeldoorn, Marcel Karperien. Proliferation restrictive micro-environments for beta cell encapsulation. In preparation 
Sieger Henke*, Tom Kamperman*, Jacqueline Plass, Rong Wang, Pieter J Dijkstra, Jeroen Leijten, Marcel Karperien. Biofabrication of core-shell hydrogels as microbioreactors for controlled cell aggregation. In preparation

Sieger Henke*, Tom Kamperman*, Niels Ruiterkamp, Bram Zoetebier, Rong Wang, Pieter J Dijkstra, Jeroen Leijten, Marcel Karperien. Engineering enzymatically crosslinkable microgels using hydrogen peroxide nanoemulsion. In preparation

Tom Kamperman*, Sieger Henke*, Niels Ruiterkamp, Pieter J Dijkstra, Marcel Karperien, Jeroen Leijten. Centering Single Cells in Spherical Microgels by Uncoupling On-chip Emulsification and enzymatic crosslinking using hydrogen peroxide diffusion. In preparation

Tom Kamperman, Sieger Henke, Martin Neubauer, Andreas Fery, Marcel Karperien, Jeroen Leijten. Engineering 3D Single Stem Cell Microniches with In Situ Tunable Stiffness. In preparation

Tom Kamperman, Sieger Henke, Cindy Kelder, Michelle Koerselman, Pieter J Dijkstra, Jeroen Leijten, Marcel Karperien. In Situ Modifiable Multifunctional Hydrogel for Advanced Tissue Engineering. In preparation

Yao Fu, Sanne Both, Sieger Henke, Jeroen Leijten, Marcel Karperien. Chondroinduction in co-culture of micro aggregated chondrocytes and mesenchymal stromal cells. In preparation

\section{Abstracts selected for poster presentation}

Sieger Henke, Jeroen Leijten, Giulia Marchioli, Evelien Kemna, Albert van den Berg, Pieter J Dijkstra, Aart van Apeldoorn, Marcel Karperien. Microgels for Tissue Engineering. The Netherlands Society for Biomaterials and Tissue Engineering annual meeting 2012.

Sieger Henke, Jeroen Leijten, Evelien Kemna, Albert van den Berg, Pieter J Dijkstra, Aart van Apeldoorn, Marcel Karperien. Microfluidic Encapsulation of Beta Cells: Comparing the Suitability of Enzymatic, Ionic and Photo Crosslinking Hydrogels. The $4^{\text {th }}$ joint EPITA and AIDPIT winter symposium, 2014, Igls, Austria. 
Sieger Henke, Jeroen Leijten, Evelien Kemna, Albert van den Berg, Pieter J Dijkstra, Aart van Apeldoorn, Marcel Karperien. Enzymatically crosslinked Dextran-Tyramine micro hydrogels for improved beta cell function. $1^{\text {st }}$ International Conference on Micro \& Nanofluidics, Fundamentals and Applications, 2014, Enschede, The Neterlands.

Sieger Henke, Jeroen Leijten, Albert van den Berg, Pieter J Dijkstra, Aart van Apeldoorn, Marcel Karperien. Microfluidic production of tissue engineered islets of Langerhans: microgel functionalization using biomimetic elements. RIMLS Symposium 2014 New Frontiers in Regenerative Medicine, Nijmegen, The Netherlands

Sieger Henke, Jeroen Leijten, Tom Kamperman, Rong Wang, Albert van den Berg, Pieter J Dijkstra, Aart van Apeldoorn, Marcel Karperien. Droplet microfluidics for the encapsulation of beta-cells in microgels of natural polysaccharides conjugates. The Netherlands Society for Biomaterials and Tissue Engineering annual meeting 2014

\section{Abstract selected for oral presentation}

Sieger Henke, Jeroen Leijten, Evelien Kemna, Albert van den Berg, Pieter J Dijkstra, Aart van Apeldoorn, Marcel Karperien. Enzymatically crosslinked Dextran-Tyramine micro hydrogels for improved beta cell function. The Netherlands Society for Biomaterials and Tissue Engineering annual meeting 2013 


\section{Curriculum Vitae}

Sieger Henke was born on the $6^{\text {th }}$ of November 1986 in Beuningen (Gld), The Netherlands. He obtained his gymnasium degree (nature \& technology and nature \& health) at the "Christelijk Gymnasium Beyers Naudé" in Leeuwarden. He studied Biomedical Engineering at the University of Twente in Enschede from 2005, obtained his bachelor's degree in 2009, and obtained his master's degree in November 2011. During his graduation project he started with the development of microfluidically formed microgels. He continued the development of microgel technology during his PhD under the supervision of Dr. Jeroen Leijten, Dr. Aart van Apeldoorn and Prof. Dr. Marcel Karperien. The results of his research on microgel technology for improved beta cell transplantation are presented in this thesis. 


\section{Acknowledgements}

It all started during my graduation project where I decided to try a microfluidic solution to create micro aggregates. I would like to thank Jeroen, especially for helping getting things started, the weekly meetings, endless discussions and never ending stream of idea's. I want to thank Aart to allow me to do my PhD in the Islet group and the DCTI project. I would like to thank Marcel for giving me the opportunity to perform my research in his group. Thank you for helping, writing and advice.

Then I would like to thank Tom. It was always fun working together, throughout the week, but also Friday afternoon trying new things. You might be the most down-to-earth person I know. Thank you for working together! I want to thank Milou and Brenda for drinking tea and coffee, lab troubleshooting, sharing complaints about everything that's not going right, and having discussions about work and non-work related matter.

I would like to thank my officemates with who I shared the office for most of my PhD. Rong and Leilei for introducing me to the Chinese culture, Jetse for the nice chats about everything and nothing, Jan for brainstorming about research or just about everything, and off course for organizing the "borrels". Then I would like to thank Giulia, Janneke, Mijke and Don of the islet group for discussing and improving our research. I also would like to thank the current and previous members of the DBE group, for helping in the lab, or just for a nice chat during work. Especially I would like to thank Jacqueline and Sanne for always listening, helping where needed and trying to keep everything up and running. And Ingrid for being always available to help to get organizationally and office related stuff done. I would like to thank the students I supervised, Jan, Laura, Roelof, Alise and Pinak. It was not only very nice to supervise you, I learned a lot from you and I appreciate your input.

I would like to thank Evelien and Johan from the BIOS group for helping with my first steps in chip design and manufacturing of the chips. Your input and practical help was essential to get the first prototypes working. And I thank Marc from the BST group for helping creating lab space for the setup and practical things in the chemistry labs. Andries thanks for helping me with my first steps in science during my bachelor research. From the LUMC I would like to thank Eelco 
and Francoise for giving me the opportunity to perform the in vivo experiments, and especially Annemieke and Maaike to help me with arranging everything and the actual hands-on work.

I want to thank Marco and Bjorn, my housemates during most of my PhD. I could always talk to you when things did not go well. During the time we lived together we shared a lot of good conversations, beers, pizza's, bbq's and other good food. Gert, I enjoyed our moments of drinking coffee and meanwhile our discussions about healthcare or random other things. Pascal, I really appreciated our discussions and chats and most of all your way of making conclusions of things we discussed and putting things into perspective.

I would like to thank everyone that contributed in any way. I might forget someone, so if you are reading things and you miss your name, thanks for helping me! Then a special word to my family, you introduced me in the fields of technology and healthcare and learned me already as a young boy to discover things. Thank you for your continuous support! And finally I would like to thank Jacobien. You had to listen to my complaints about everything and nothing, without exactly understanding what I was doing. You kept calm and kept supporting me!

Sieger Henke October 2016 
